Portland State University

PDXScholar

Fall 1-27-2017

\title{
Building a Multivariable Linear Regression Model of On-road Traffic for Creation of High Resolution Emission Inventories
}

James Eckhardt Powell

Portland State University

Follow this and additional works at: https://pdxscholar.library.pdx.edu/open_access_etds

Part of the Physics Commons

Let us know how access to this document benefits you.

\section{Recommended Citation}

Powell, James Eckhardt, "Building a Multivariable Linear Regression Model of On-road Traffic for Creation of High Resolution Emission Inventories" (2017). Dissertations and Theses. Paper 3415.

https://doi.org/10.15760/etd.5313

This Thesis is brought to you for free and open access. It has been accepted for inclusion in Dissertations and Theses by an authorized administrator of PDXScholar. Please contact us if we can make this document more accessible: pdxscholar@pdx.edu. 
Building a Multivariable Linear Regression Model of On-road Traffic for Creation of High Resolution Emission Inventories

by

James Eckhardt Powell

A thesis submitted in partial fulfillment of the requirements for the degree of

\author{
Master Of Science \\ in \\ Physics
}

Thesis Committee:

Christopher Butenhoff, Chair Linda George

M. Aslam K. Khalil

Andrew Rice

Portland State University

2016 


\begin{abstract}
Emissions inventories are an important tool, often built by governments, and used to manage emissions. To build an inventory of urban $\mathrm{CO}_{2}$ emissions and other fossil fuel combustion products in the urban atmosphere, an inventory of on-road traffic is required. In particular, a high resolution inventory is necessary to capture the local characteristics of transport emissions. These emissions vary widely due to the local nature of the fleet, fuel, and roads.

Here we show a new model of Average Daily Traffic (ADT) for the Portland, OR metropolitan region. The backbone is traffic counter recordings made by the Portland Bureau of Transportation at 7,767 sites over 21 years (1986-2006), augmented with PORTAL (The Portland Regional Transportation Archive Listing) freeway traffic count data. We constructed a regression model to fill in traffic network gaps using GIS data such as road class and population density. An EPA-supplied emissions factor was used to estimate transportation $\mathrm{CO}_{2}$ emissions, which is compared to several other estimates for the city's $\mathrm{CO}_{2}$ footprint.
\end{abstract}


DEDICATION

To my family: Carol L. Linné and Paul Y. Irvin, John G. Powell and Debby E. Powell, Ryan A. Powell and Kristin E. Bowen, Emmett J. Powell and Miles V.

Powell. You are my inspiration. 


\section{ACKNOWLEDGMENTS}

For our wonderful software libre stack, we thank the developers of GNU emacs, GNU/Linux, gcc, Debian and CentOS, Sun Grid Engine (SGE), GRASS GIS, PostgreSQL, R [1], Lattice [2], and cs2cs. A big thank you to Chad Sarni of PSU Physics for downloading the PORTAL data for us, and to William Garrick of PSU Research Computing for translating the TDAT into SQL. Kristin Tufte has been patient answering many queries we've had about PORTAL. Jamie Throckmorton and Tom Jensen of PBOT have unlocked the Bureau of Transportation's methods for us. Dr. Andrew Rice has helped us on many occasions by providing interesting avenues for new research and by his keen insight into the research problem. Dr. Aslam Khalil has been a steady support through some rocky periods. Dr. Khalil has maintained a big picture view of the field for me throughout, and he has my gratitude for introducing me to my advisor, Dr. Chris Butenhoff. I thank Dr. Butenhoff for his almost superhuman patience with my work, his insanely great science skills, and his ability to reign in my sometimes wild speculation thereby directing this research to the conclusion you will read about here. 
Table Of Contents

\begin{tabular}{lll}
\hline 1 & Introduction & 1
\end{tabular}

1.1 Background . . . . . . . . . . . . . . . . . . . . . . 1

1.1.1 The Big Picture . . . . . . . . . . . . . . . . . . . 7

$\begin{array}{lll}2 & \text { Methods } & 11\end{array}$

2.1 Overview . . . . . . . . . . . . . . . . . . . . . . . . . . 11

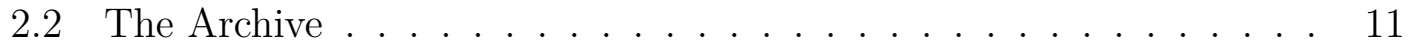

2.3 Data Understanding . . . . . . . . . . . . . . . . . . . . . . . . . . 12

2.3 .1 TDAT . . . . . . . . . . . . . . . . . . 12

2.3 .2 PORTAL . . . . . . . . . . . . . . . . 15

2.4 Data Cleaning and Reduction . . . . . . . . . . . . . . . 15

2.4.1 Data Quality Problems Common to Both Archives . . . . . 17

$2.4 .2 \quad$ TDAT-specific Data Quality Problems . . . . . . . . . . . . 18

$2.4 .3 \quad$ PORTAL-specific Data Quality Problems . . . . . . . . . . . 19

2.5 The Use of the Computer for Reproducibility and Automation . . . 19

2.6 Statistics . . . . . . . . . . . . . . . . . . . . . . . 22

2.7 Data Preparation . . . . . . . . . . . . . . . . . . 22

2.7 .1 TDAT . . . . . . . . . . . . . . . . . . 22

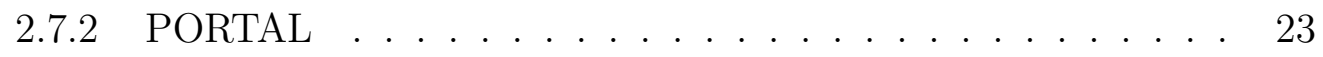

2.8 Deriving a Canonical Model of ADT . . . . . . . . . . . . . . . 23

$2.8 .1 \quad$ Selection of Land Use Regression Variables . . . . . . . . . . 24 
2.8.2 Establishing the Log-normality of the Counts . . . . . . . . 24

2.8.3 Using Multiple Linear Regression to make a Linear Model . 31

$2.8 .4 \quad$ Obtaining VMT . . . . . . . . . . . . . . . . . . . 31

2.8 .5 The Multiple Linear Regression . . . . . . . . . . . . . . . . 31

2.9 Preparation for Massively Parallel LUR . . . . . . . . . . . . . . . . 32

2.10 Massively Parallel LUR . . . . . . . . . . . . . . . . . . . . . . . . . 33

2.10 .1 Population Density . . . . . . . . . . . . . . . . . . . . 33

2.11 Exploration . . . . . . . . . . . . . . . . . . . . . . 33

2.12 Hypothesis Testing . . . . . . . . . . . . . . . . . . . . 33

2.12.1 Significance of Terms in the Linear Regression . . . . . . . . 36

2.12 .2 Normally Distributed Residuals . . . . . . . . . . . . . . . . 36

2.12 .3 Weekend Effect . . . . . . . . . . . . . . . . . . . . . . . . 36

2.12 .4 Bimodal Distribution (Rush Hours) . . . . . . . . . . . . . . 37

2.12 .5 Seasonal Effect . . . . . . . . . . . . . . . . . . . . 37

2.12 .6 Moss Nitrogen Density Experiment . . . . . . . . . . . . . . 37

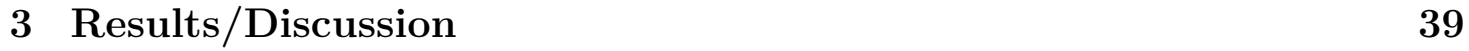

3.0 .7 Tables of Outliers . . . . . . . . . . . . . . . . . . . . . 39

3.0.8 H01: Our ADT values compare well with other published estimates. . . . . . . . . . . . . . . . 41

3.0 .9 H02: High (low) moss nitrogen concentration correlates with high (low) traffic counts. . . . . . . . . . . . . . . . . 42

3.0.10 H03: The Shaprio-Wilk test will confirm that the count data are $\log$-normal. . . . . . . . . . . . . . . . . . . . . . . . . . 42

3.0.11 H04: Linear regression is appropriate for the traffic count data + our Land Use Regression (LUR) statistics . . . . . . 45 
3.0.12 H05: Observing that the residuals of the linear regression are homoscedastic . . . . . . . . . . . . . . . 45

3.0.13 The linear regression results. . . . . . . . . . . . . . . . . 46

3.0.14 H06: the linear regression is statistically significant . . . . . 46

3.0.15 H07: a linear regression can reproduce trends in the original traffic data .................. . . 46

3.0.16 H08: there are few outliers in the cleaned data. . . . . . . . 49

3.0.17 H09: Rush hours will produce a bimodal trend in the diel, and weekends will be highly attenuated in counts. . . . . . . 49

3.0 .18 H10: Small seasonal differences are expected. . . . . . . . . . 52

3.0.19 H11: Our emissions inventory (EI) compares well with other, independent estimates . . . . . . . . . . . . . . 52

3.1 Limitations . . . . . . . . . . . . . . . . . 53

$3.1 .1 \quad$ Uncertainties . . . . . . . . . . . . . . . 54

$\begin{array}{lll}4 & \text { Conclusion } & 56\end{array}$

$\begin{array}{lll}5 & \text { Future Work } & 58\end{array}$

\begin{tabular}{|cc}
\hline Bibliography & 63
\end{tabular}

\begin{tabular}{ll}
\hline A Appendices & 71
\end{tabular}

A.1 Data Preprocessing Steps . . . . . . . . . . . . . . . . 71

A.2 Data Cleaning Pipeline Flowchart . . . . . . . . . . . . . . . . . . . 71

A.3 Archival Process . . . . . . . . . . . . . . . . . . . . 76

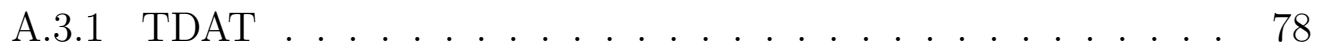

A.3.2 PORTAL . . . . . . . . . . . . . . . 82

A.3.3 The TDAT Schema - a Finding Aid . . . . . . . . . . . . . . 84 
A.4 Data Cleaning Details . . . . . . . . . . . . . . . . . 84

A.4.1 TDAT . . . . . . . . . . . . . . . . . 84

A.5 TDAT Road Class Assignment - Technical Details . . . . . . . . . . 87

A.5.1 Our effort to use a spatial join fails due to great disparities between road maps . . . . . . . . . . . . . . . . . . 88

A.5.2 A key is found that enables road class assignment . . . . . . 89

A.5.3 An anomaly is found that casts doubt . . . . . . . . . . . . 91

A.6 Source code . . . . . . . . . . . . . . . . . . . . . . . . . . . . . 94

A.6.1 Tiny Toy Data For Sample Calculations . . . . . . . . . . . 94

A.6.2 Adapt PORTAL to the TDAT data structure . . . . . . . . 96

A.6.3 Weekend Test . . . . . . . . . . . . . . . . . . . . . . 100

A.6.4 Diagnostic Plots for the Log Transformation. Shapiro-Wilks. 101

A.6.5 Join the raw counts with our pseudo-LUR variables. . . . . . 102

A.6.6 Reduce the data to one row for each day. . . . . . . . . . . . . 103

A.6.7 Translate road class (RC) identifiers to name. . . . . . . . . 104

A.6.8 Make a diel graph . . . . . . . . . . . . . . . . . . 105

A.6.9 Make a random subset of the raw data for validation purposes.106 A.6.10 Run a stepwise linear regression on the model subset. . . . . 107 A.6.11 Count days for each outlier class . . . . . . . . . . . . . 108

A.7 Source Code for the Pipeline . . . . . . . . . . . . . . . . 108

\begin{tabular}{ll}
\hline Interesting Statistics & 109
\end{tabular}

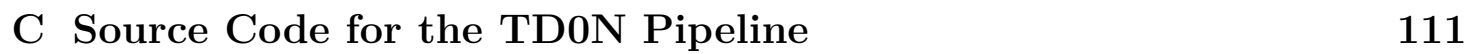


List of Tables

2.1 A side-by-side comparison of the very different attributes of the TDAT and PORTAL archives. . . . . . . . . . . . . . . . . . . . . . 12

2.2 The result of our brainstorming effort, informed by research, about factors that could sensibly affect traffic counts in the city. . . . . . . 26

$3.1 \quad$ A table summarizing the hypotheses tested in this research. . . . . 40

3.2 The types of outliers located in TDAT with an approximate number of location/days affected. The id column is a unique identifier assigned to the outlier type. . . . . . . . . . . . . . . . . . 40

3.3 The types of outliers found in PORTAL with an approximate number of location/days affected. The id column is a unique identifier assigned to the outlier type. . . . . . . . . . . . . . . . . . . . 41

3.4 Coefficients from the linear regression. The units are in the natural $\log$ of the ADT. The significance codes are 0 (***) $0.001^{(* *)} 0.01^{\text {(*) }}$ 0.05 .. The multiple $\mathrm{R}^{2}$ is 0.8904 . . . . . . . . . . . . . . . . . . . . 47

A.1 Represented road classes in the toy study area, with color codes assigned. . . . . . . . . . . . . . . . . . 81

A.2 Portal freeway coverage. . . . . . . . . . . . . . . . . . 84 
List of Figures

1.1 Factors determining urban $\mathrm{CO}_{2}$ concentrations, after 8 , Figure 2]. Photograph by the author. . . . . . . . . . . . . . . . . 2

1.2 Portland, OR GHG emissions by sector $|10| .$. . . . . . . . . . . . . 3

1.3 Conceptual model of the Circoscope research. . . . . . . . . . . . . 8

2.1 A map of a part of the study area showing colors indicating road class. The colors are as given in Table A.1. . . . . . . . . . . . . 13

2.2 TDAT count sites in the Portland Metro Area. Red dots are sites. The yellow line is the Urban Growth Boundary. The blue is the Willamette and Columbia rivers, and the gray lines are streets. Data sources: Metro's RLIS GIS data and the PBOT TDAT2 archive. . . 14

2.3 PORTAL sites in the Portland Metro Area. Blue crosses are stations, yellow lines are freeways, and the gray area is the urban growth boundary. . . . . . . . . . . . . . . . . . . . 16

2.4 Known Traffic DATa (TDAT) counter arrangements. Numbers inside of square boxes are channels. . . . . . . . . . . . . . . . . . . . 18

2.5 A schematic of the software stack used in the research. . . . . . . . 21

2.6 Actual counts and model counts on a map of Portland, OR. Vertices are raw data, while the color of the line segments is modeled based on road class, population density, and on-ramp mileage. . . . . . . . 25 
2.7 Histograms (top) and QQ plots (bottom) for a random sample of weekday ADT, untransformed. No indication of normality is seen in these graphs. Each panel is one of the road classes. Codes (a), (b) etc. are as in Figure 3.5

2.8 Histograms (top) and QQ plots (bottom) for a random sample of weekday ADT, log-transformed. Normality is seen in these graphs. Each panel is one of the road classes. Codes (a), (b) etc. are as in Figure $\mid 3.5$

2.9 In (a), the square root of standardized residuals plotted against the fitted values for all of the raw data, without a log-transform. Observe the definite proportionality of the variance of the residuals against the fitted values. In (b), after the log-transform the residuals appear more normally distributed around zero. . . . . . . . . . . . . . . . . 30

2.10 Centroids assigned to each road segment in RLIS streets. . . . . . . 32

2.11 A population density map of the Portland, OR region. The rivers are a composite of RLIS's fine Metro-area base layer and TIGER's coarser state-wide coverage of waterways. The data are compiled at the level of the census tract, which is approximately the scale of a neighborhood. . . . . . . . . . . . . . . . . . 34 


\section{LIST OF FIGURES}

2.12 A box plot of the combined PORTAL and TDAT traffic count data after cleaning. $\mathrm{N}=34,308$ ADT values were used, each representing one day of counting at one site. The boxes represent the interquartile range: $25 \%$ of the data lie below the bottom line and $25 \%$ lie above the top line, while the interior line shows the median. Adjacent values are represented by "whiskers" (dashed lines) extending off of the top and the bottom of the box. Calculation of the adjacent values is described in $[46$, p. 242]; they show the approximate range of the data. Extreme values outside this range are drawn as circles.

2.13 A map showing all 81 of the moss experiment sample sites. Red crosses are sample sites. Counties are named. . . . . . . . . . . . . 38

$3.1 \quad$ Moss collection sites outside of our ADT study area. . . . . . . . . 43

3.2 Plot of nitrogen content in moss vs. traffic counts. . . . . . . . . . . 44

3.3 The actual count data in the set held back during model creation is plotted against the values for these counts predicted by the linear model. The equation of the line is $\mathrm{y}=-0.08613+1.00799 \mathrm{x}$. The $\mathrm{r}^{2}$ is $0.90 .11,463$ data points are plotted. . . . . . . . . . . . . . . . . 48

3.4 Leverage of the points used in the regression, with Cook's Distance marked. . . . . . . . . . . . . . . . 50 
3.5 A plot showing the diurnal (diel) trends in the data. Each panel graphs

all of the available data, as counts per day vs. the time of day (00:00-23:00). The

all or the available data, as counts per day vs. the time of day (00:00-23:00). The

blue colored dots are a single datum for each hourly binned count made on a week

day, red are the same but for counts made on weekends only. (a) = "In the area

maintained by the City of Portland only". (b) = "Private right-of-way exists". (c)

$=$ "With rapid transit". (d) = "To local street connector (Portland only, subarea $=$

'P')". (e) = "Named, but without valid addressing (Clackamas County only, subarea =

'C')". (f) = "Unnamed and without valid addressing (Clackamas County only, subarea

$=$ 'C')". (g) = "With valid address range and street name (in the area maintained by

the City of Portland only)". (h) = "With NO Valid Address Range or Street Name

(in the area maintained by the City of Portland only)". (i) = "No private right-of-way

exists". (j) = "Passable by emergency vehicles (e-911) only (in the area maintained by

the City of Portland only)". . . . . . . . . . . . . . . . . . . . . . . . . . 51

5.1 Conceptual model of the WRF components to the research. . . . . . 59

$5.2 \quad$ A $1 \mathrm{~km}$ resolution gridded $\mathrm{CO}_{2}$ emissions inventory for Portland, OR developed using our model. We presented this result at the GEIA conference in Beijing, December 2015. The EI shows the expected high intensity of emissions along the east-west I-84 corridor (straight line, top center) and the north-south I-205 corridor (straight line, center right), zero emissions on an all-river pixel (the white pixel at bottom left), and fairly high emissions over downtown (top-left). The units are $\mathrm{MT}$ of $\mathrm{CO}_{2}$ /year. . . . . . . . . . . . . . 60

A.1 TD0N pipeline, 1/4. . . . . . . . . . . . . . . . 72

A.2 TD0N pipeline, $2 / 4 . \ldots \ldots \ldots \ldots \ldots \ldots$

A.3 TD0N pipeline, $3 / 4 . \ldots \ldots \ldots \ldots \ldots \ldots$

A.4 TD0N pipeline, $4 / 4 . \ldots \ldots \ldots \ldots \ldots \ldots \ldots$ 
A.5 A UML diagram of the namelist database. We created this finding aid to guide our manual and automated research into the archives. . $\quad 77$

A.6 A day of counting at one site in the center of the city. . . . . . . . . 79

A.7 Map of circles proportional to the number of times PBOT decided to open a count at the site. Note the big circles all focused on the downtown area and linear patterns of counts opened along major $\mathrm{N} / \mathrm{S}$ and $\mathrm{E} / \mathrm{W}$ corridors. North is up. The Willamette river can be seen as a lacuna opening in the top-left of the map. A large forested park is immediately to the west of the river at the topleft, represented as another lacuna. The remaining empty space represents the Columbia river to the north, and other jurisdictional boundaries to the east, south, and west. . . . . . . . . . . . . . 80

A.8 The TDAT schema diagram, part 1. . . . . . . . . . . . . . . . . 85

A.9 The TDAT schema diagram, part 2. . . . . . . . . . . . . . . 86

A.10 An overlay map of the area around SE 29th and SE Waverleigh. North is up. Red lines are road segments from the PBOT segments. Blue lines are road segments from RLIS streets. Green lines represent the software's best attempt to spatially join RLIS streets with PBOT segments. Note the remarkable degree of divergence between the two maps, far too great for an automated system to reconcile them. . . . . . . . . . . . . . . . . . . . . . . 89

A.11 Map showing one of the TDAT count sites where it is not possible without ground truthing to identify the road class of the site. RLIS streets includes two roads with different road classes in the immediate vicinity. . . . . . . . . . . . . . . . . . . . . . . . . . 93 
LIST OF FIGURES 
"The evidence is incontrovertible: global warming is occurring"(American Physical Society, Policy Statement 7.1, 2007-11-18)

"Warming of the climate system is unequivocal" [3]

Global warming has already given us a warmer ocean/atmosphere system, less ice, and a sea level that has and will continue to rise [3, p. 4]. $\mathrm{CO}_{2}$ in the atmosphere has been rising due to human activities, and the rise since 1750 has created a situation where anthropogenic $\mathrm{CO}_{2}$ has enhanced the radiative forcing $(\mathrm{RF})$ of the greenhouse 1 effect by a factor of 1.68 [1.33 to $2.03 \mathrm{~W} \mathrm{~m}^{-2}$ ] compared to 1750 levels. $\mathrm{CO}_{2}$ is thus the "elephant in the room," being the anthropogenic gas most responsible for global warming [3, p. 13].

Consequently, great energy has been and will continue to be focused on cutting, drastically, humanity's $\mathrm{CO}_{2}$ footprint. Only two avenues are available to us: mitigation and sequestration. Sequestration, while a favorite of governments, is so far limited by the lack of incentive for power companies to pay for it, and difficulties with storage of the sequestered $\mathrm{CO}_{2}$ [4. We believe that mitigation is a key part of the anthropogenic $\mathrm{CO}_{2}$ emissions problem and that emissions inventory (EI)s are a primary tool in our effort to find an effective means of mitigation.

The global change research community has been constructing high resolution

\footnotetext{
${ }^{1} \mathrm{~A}$ misnomer: greenhouses are primarily heated by convection, not radiation.
} 


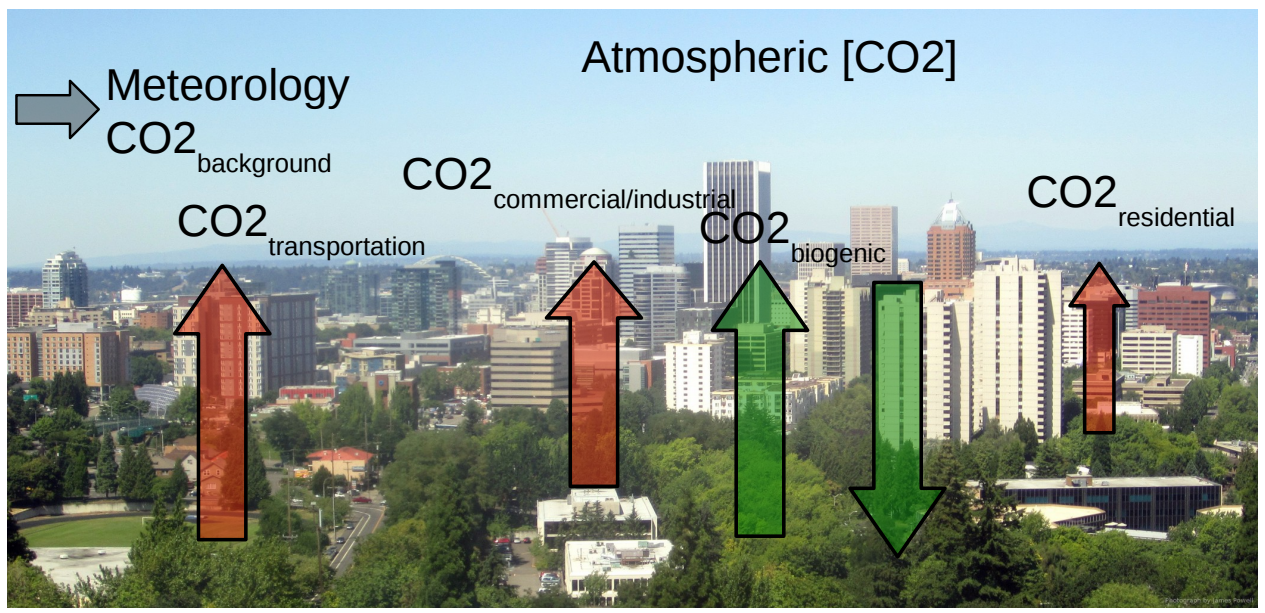

Figure 1.1: Factors determining urban $\mathrm{CO}_{2}$ concentrations, after [8, Figure 2]. Photograph by the author.

$\left(<10 \mathrm{~km} \times 10 \mathrm{~km}\right.$ resolution) EI of $\mathrm{CO}_{2}$ for several years [5]. The call for this type of EI is to improve policy making for climate change mitigation and to help scientists to close the carbon budget at small scales. Our field was recently described as "the nascent field of modeling and observing urban carbon pools and flows" [6]. Observers see the possibility that "an urban research campaign ... [that] combines surface observations [with] ... high-resolution flux estimation ... could transform carbon cycle science" (ibid). Meta-analyses have abundantly identified "the importance of using localized data for accuracy in terms of emissions estimates" [7], however such data and the expertise needed to analyze them are prohibitively expensive in many cases.

The fluxes of $\mathrm{CO}_{2}$ in a city are complex, including biogenic, residential, commercial/industrial, and $\mathrm{CO}_{2}$ from transportation and that blown in by winds in a regional mixing. Figure 1.1 shows these sources and sinks.

The biggest significant change, one that really does alter what it is like to be human, may well be modern communication and transportation" [9. p. 249, emphasis mine] 


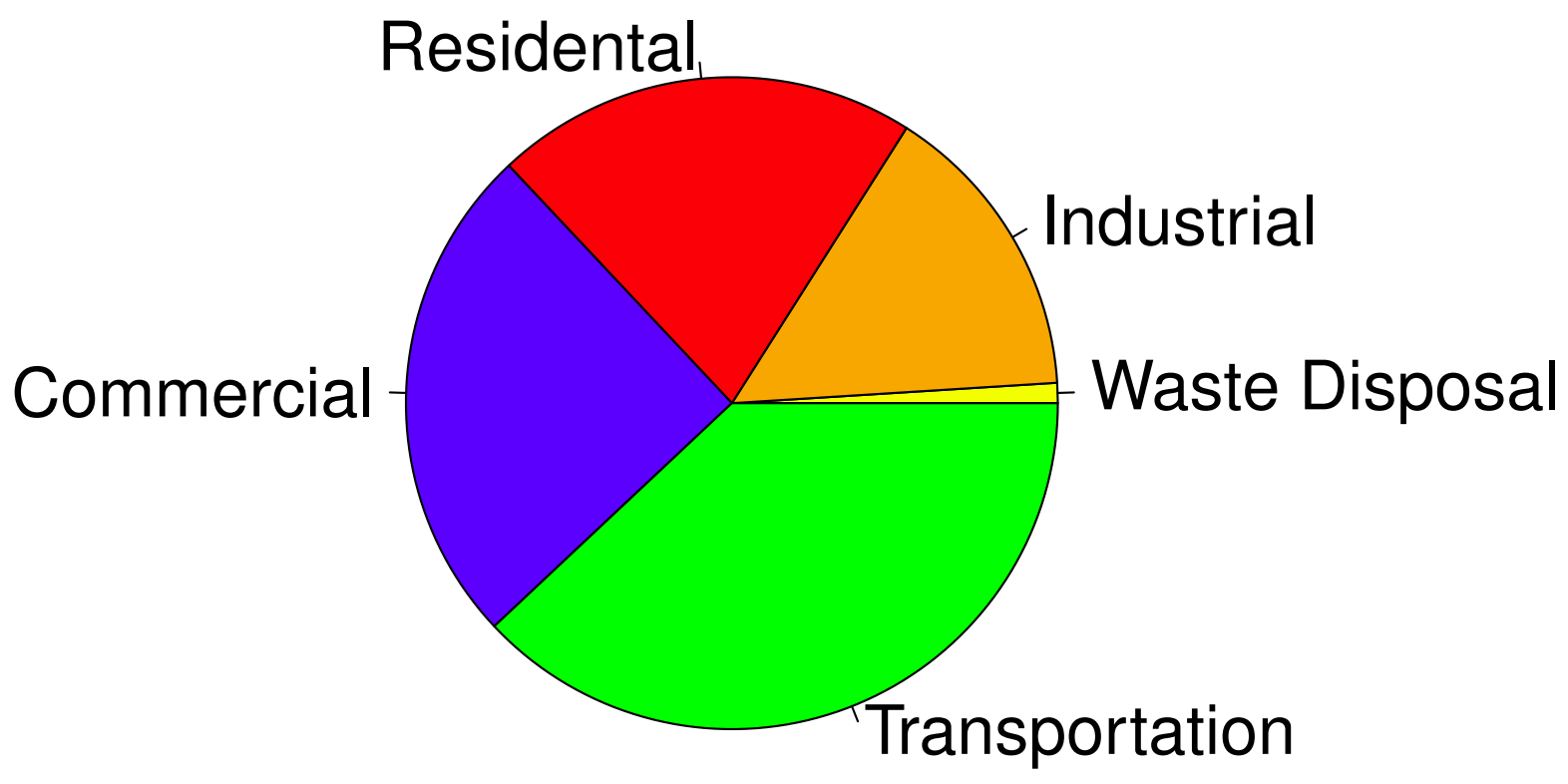

Figure 1.2: Portland, OR GHG emissions by sector [10].

Figure 1.2 demonstrates the importance of the transportation sector in $\mathrm{CO}_{2}$ emissions for Portland. We believe, for reasons discussed throughout, that urban on-road transport is where the major focus for $\mathrm{CO}_{2}$ emissions mitigation should be.

In 2014 [6] conducted a broad survey identifying the way forward from the current, weak knowledge regarding the pathway to a sustainable, low-carbon future for our cities. The situation is complex in the extreme; carbon use trajectories vary widely between developed and developing countries, stakeholders are many and powerful, and equity questions are strongly in play.

It will be possible to plot a sustainable course by improving our understanding through "integrated, coproduced approaches to urbanization" (ibid.), combining expertise of engineers, social scientists, and natural scientists. A repeated theme in this work is the importance of "researchers and practitioners who create a thorough quantification of city-level carbon emissions" (ibid).

Here we show a novel technique of making a high resolution EI by combining 
two extensive and local data sources for transportation with several governmentcultivated yet still local data sources for land-use (where the term land-use is taken loosely to include factors like population density and road density). We report here the methods and results for developing a high-resolution, high-accuracy $\mathrm{CO}_{2}$ emissions inventory for the North American city of Portland, OR. This is the first work to our knowledge that examines raw traffic count data, a primary source; preceding efforts have relied on state-reported numbers for VMT, which were shown in [11] to be quite inaccurate for some states.

Hundreds of governments of all scales, world-wide are working to reduce $\mathrm{CO}_{2}$ emissions, including for example the state of California [12], the city of Portland, OR [10], and a coalition of hundreds of cities in Local Governments for Sustainability (ICLEI) [6], and many nations under the Kyoto Protocol which was put forward in 1997 by the United Nations Framework Convention on Climate Change (UNFCCC). The Paris talk of 2016 resulted in a commitment by the US to reduce $\mathrm{CO}_{2}$ emissions to "26-28 percent below 2005 levels by 2025" 13.

Beyond voluntary efforts such as these, binding agreements between states are emerging, and these are likely to have far-reaching economic impact. Such agreements will require a process known as monitoring reporting and verification (MRV) 14. p. 8423]. Emissions inventories are expected to be a primary means of demonstrating compliance, but the accuracy of EI generally are as large or greater than the expected emissions reductions.

Evidence is building that deep cuts in urban emissions will be required to mitigate the worst (and potentially catastrophic) effects of global warming [15, p. 486] [10] For example, in 2008 Wheeler wrote that "a small but growing number of U.S. states and cities have adopted long-term goals that would reduce $\mathrm{CO}_{2}$ equivalents nearly $80 \%$ below the 2000 level by 2050." [15].

Policy makers are interested in $\mathrm{CO}_{2}$ emissions mitigation as a source of options 


\subsection{BACKGROUND}

(primarily cap and trade) in the face of potentially catastrophic climate change. Emissions are of interest because sequestration options appear highly limited [16. An accurate EI is a required vehicle for proving the effectiveness of emissions mitigation policy and also a useful tool for exploring mitigation options.

Carbon cycle research includes two distinct important elements: a terrestrial sink that is hard to quantify (the biosphere) and a fossil fuel source that is well known. In inversion studies, the latter is often used to constrain the former, and even slight inaccuracies in spatial or temporal inventories of $\mathrm{CO}_{2}$ emissions from fossil fuels result in uncertainty in estimations of Net Ecosystem Exchange (NEE) 17 18.

The global change research program's attention has rightly fallen to the urban scale $[6]$, because recently the balance of humanity became urbanized and the majority of global $\mathrm{CO}_{2}$ emissions are urban. Past studies have produced EI on a global or national scale, however such work is not useful for understanding urbanscale sources and sinks because an EI commensurate with the urban scale is needed.

In the United States (US), where there is no national policy ${ }^{2}$, the scale at which policy is being made is often urban.

Cities have been shown to be the source of most of the growth in on-road $\mathrm{CO}_{2}$ emissions in recent decades [11], and cities have another reason to mitigate: cost savings. One study concluded that city managers are motivated by cost savings more than public opinion, and that cities can save money in some cases through mitigation 20$]$.

Cities are also important for their activism (ICLEI) and because there is a governance advantage at work. We generally find that policymakers at the urban level have the ready ability to translate decisions into action especially in the

\footnotetext{
${ }^{2}$ In 2009 the EPA established a policy of maintaining an emissions inventory of Greenhouse Gas (GHG) from major emitters in the US [19], indicating some further regulation may be forthcoming.
} 
transport realm (Leonor Tarrasón, in a presentation at GEIA 2015).

As humanity works to curb $\mathrm{CO}_{2}$ emissions, Integrated Assessment Model (IAM)s are a useful tool for categorizing the emitters. However, global IAMs ignore urbanlevel work, where much interesting effort is going into urban planning and urban mode shift work which will impact mitigation. Our high-resolution urban model will capture this effect and enable these local efforts to be a part of the conversation about $\mathrm{CO}_{2}$ mitigation 21.

Globally, the transport sector was found to be the source of $23 \%$ of $\mathrm{CO}_{2}$ emissions in the year 2010 [22, p. 4]. On-road transport is $81 \%$ of that quantity 23. We find that on-road transport (that is, not air, rail, or water) is the largest category of Greenhouse Gas (GHG) emissions nationwide in the USA [19], and $38 \%$ of GHG emissions in Portland, OR [10]. The transport sector overall was

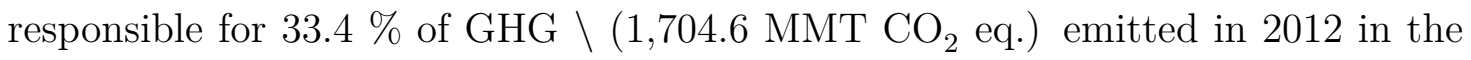
US, the last year reported.

This work differs from the usual approach in that we draw from primary sources: the Portland Bureau of Transportation (PBOT) Traffic DATa (TDAT) archive and the Portland Regional Transportation Archive Listing (PORTAL) archive. there is another study which used road-segment level Vehicle Miles Travelled (VMT) to avoid any requirement of down scaling by proxy [11, p. 5000], our product offers a direct, local and bottom-up result.

We also see an opportunity to test some problems related to urban planning. Portland is an unusually planned city, unique in some ways: the only city in the United States with an elected metropolitan authority whose reach extends well beyond the city limits, and in 2011 the city rated 5th in the nation (after Denver, New York, Los Angeles, and Boston) for "the best combination of public transportation investment, ridership, and safety" 3

\footnotetext{
${ }^{3}$ http://www.usnews.com/news/articles/2011/02/08/10-best-cities-for-public-transportation
} 


\subsection{BACKGROUND}

So we see that matters of scientific interest (close the carbon cycle), civic interest (build a sustainable city), and international law (treat with others to avoid a tragedy of the commons) all demand much higher accuracy $\mathrm{CO}_{2}$ emissions inventories, and this is the novel contribution of our work.

Our effort has produced an emissions inventory of high resolution and accuracy for the City of Portland, Oregon, in the United States of America, for $\mathrm{CO}_{2}$ emissions from on-road transport. Our model, circoscope, is bottom-up and based on traffic counters which are believed to be a very reliable source of information, measured at $98 \%$ accurate [24].

We initiated the project in 2011 in the full understanding that a unique resource, the TDAT archive, existed and could, no doubt, be used to constrain the city's emissions inventory for $\mathrm{CO}_{2}$. The conceptual model in Figure 1.3 was developed early and still guides our work.

We were also aware that the PORTAL group had been collecting counts on all of the region's freeways (restricted roads) for nine years 25]. Since TDAT has no data for restricted roads, this PORTAL archive is vital complement allowing us to complete our picture of the region's ADT.

We designed this research then to produce a high resolution EI for the City of Portland's $\mathrm{CO}_{2}$ from on-road transport. Few other efforts have produced such high resolution EI for $\mathrm{CO}_{2}$, two examples being the Hestia inventory for Indianapolis [26 and Kevin Gately's inventory for Boston [18], and McDonald et. al.'s 2014 fuel and traffic-count based inventories for a number of US cities 27].

The discrete counts of TDAT and PORTAL were not enough for this purpose. We needed to be able to model the whole road network with a meter by meter traffic volume indicator such as ADT or VMT. Our hypothesis was that a multiple linear 
Urban $\mathrm{CO} 2$ Emissions From GIS Data

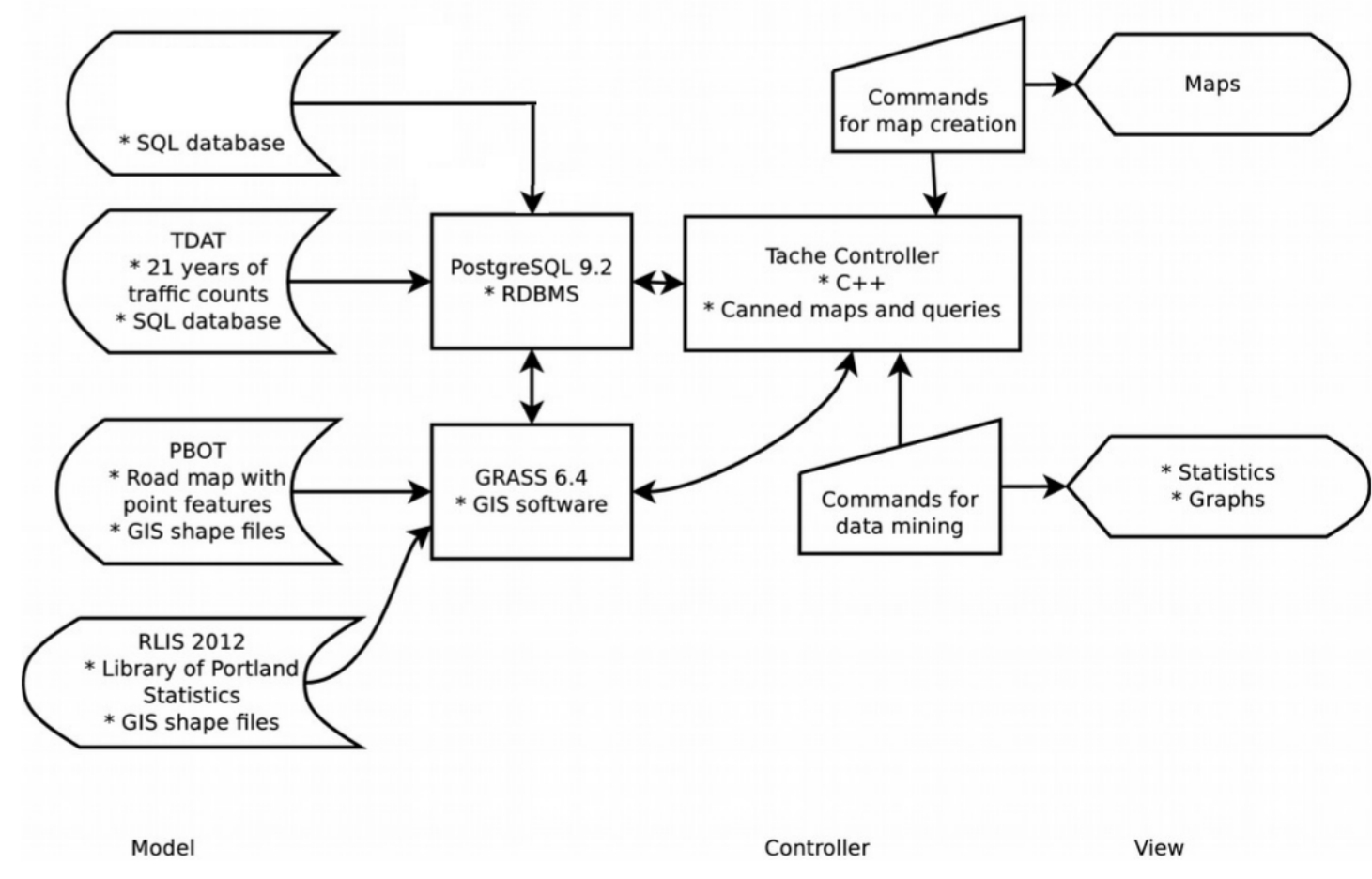

Figure 1.3: Conceptual model of the Circoscope research. 


\subsection{BACKGROUND}

regression [28, Ch. 4] based on the road class (one of 32 classes such as primary arterial, secondary arterial, minor streets) and several Geographical Information System (GIS) statistics for the region surrounding the short road segment of interest could well predict traffic counts. We wanted a model where we could ask "if I went to this road segment on this day, at this time, how many vehicles would I count going by in an hour?" and get a good answer.

When viewed at from a daily traffic count perspective, the "how many vehicles" value, called ADT, is a common and well understood measure of traffic volume. Our model depends on not only how many vehicles are traveling, but on how far they go, so we combine ADT for a road segment with the length of the road segment to obtain another common measure VMT. VMT is suitable input to emissions modeling.

To obtain an accurate model of VMT, we considered many possible drivers for ADT in a process similar to those followed by [29] and [30]. We decided to use road class, population density in circles of various radii, and freeway on-ramp mileage in circles of various radii, to build a linear model of the form

$$
\begin{aligned}
A D T & =\beta_{0}(\text { a constant intercept }) \\
& +\beta_{1} \times R C(\text { primary arterial })+\beta_{2} \times R C(\text { secondary arterial })+\ldots \\
& +\beta_{n} \times P O P D E N(100 \mathrm{~m} \text { circle })+\beta_{n+1} \times P O P D E N(200 \mathrm{~m} \text { circle })+\ldots \\
& +\beta_{m} \times O N R A M P(100 \mathrm{~m} \text { circle })+\beta_{m+1} \times O N R A M P(200 \mathrm{~m} \text { circle })+\ldots
\end{aligned}
$$

where the $\mathrm{RC}$ variables are either 1 or 0 and the $\beta$ are all constant values determined by ordinary least squares regression. The variables we selected are shown to be significant drivers of ADT.

The ADT values given by Equation 1.1 for each road segment $(\mathrm{N}=105,178)$ were multiplied by the length of the segment to give VMT. The VMT were multiplied by a nationwide average emissions factor from an EPA publication (http://www.epa. 
gov/climatechange/ghgemissions/ind-calculator.html accessed 2014-12-08)

and summed up to provide a citywide $\mathrm{CO}_{2}$ emissions rate from on-road traffic. We compared the resulting estimate for the region's $\mathrm{CO}_{2}$ footprint to Vulcan's estimate for the same region. We summed the estimate to get a single annual $\mathrm{CO}_{2}$ emissions estimate for the city and compared it to a City of Portland estimate.

We plan to go forward by using Environmental Protection Agency (EPA) MOtor Vehicle Emission Simulator (MOVES) to provide more accurate local emissions factor (EF) based on local fleet composition. This will produce a high resolution $(1 \mathrm{~km} \times 1 \mathrm{~km})$ hourly map of area $\mathrm{CO}_{2}$ emissions - a source which will be combined with a map of Vegetation Photosynthesis and Respiration Model (VPRM) 31] to give area $\mathrm{CO}_{2}$ emissions. This hourly EI will be input to the Weather Research and Forecasting (WRF) model, and samples from the model output compared to instrument recordings of $\mathrm{CO}_{2}$ concentration at three sites in an archive maintained by Dr. Andrew Rice's group at Portland State University (PSU). We expect based on the fine results for a similar project 32 to be able to reproduce the Rice record with some accuracy, thereby ground-truthing our model.

Our model then will build confidence and give tighter estimates for the city's $\mathrm{CO}_{2}$ emissions. This result will be useful in the fields of inverse modeling of $\mathrm{CO}_{2}$ sources and sinks, and separately in the discovery and monitoring of the effectiveness of mitigation measures.

We have coined the name "Circoscope" for the traffic density model. We will use the term "TD0N" somewhat interchangeably with Circoscope, although TD0N is generally used to refer to the data cleaning pipeline (the computer code) while Circoscope refers to the linear model which is a product of analyzing the clean data. 
In the course of building our model, two complicated and in some ways poorly documented databases have been processed to produce a synthetic, summary product which is both concise and accurate. Several best practice methodologies were employed to make the problem tractable. The include the methods of the archivist [33 34 35, the data miner, the software engineer 36] 37], and the statistician 38. 39.

The research described here was carried out by analyzing a variety of primary materials. Two collections of records in particular have never been used for $\mathrm{CO}_{2}$ emissions inventories before, to our knowledge. We adopt archival techniques to help the public in understanding and reproducing our work. We are comfortable that archival approaches are justified because these records, of traffic counts along roads and highways, "provide "evidence of a person or organization's activities" ,34, p. 29].

Our work primarily involved obtaining, understanding, merging, and cleaning two large raw data sets: TDAT and PORTAL. These are described in the next section, Data Understanding. 
Methods

TDAT

PORTAL

Hierarchical

Flat

Counting done deliberately, project-specific. Counters installed permanently

Many outages and special events labeled

Few (any?) special events (holidays, accidents, outages) labeled

Table 2.1: A side-by-side comparison of the very different attributes of the TDAT and PORTAL archives.

Data Understanding

To open the methods, we give an overview of the primary sources, TDAT and PORTAL. These have much in common. Both are recordings of traffic counts: the number of times a vehicle passed by on a road in a 15 minute period. The count sites in both are known by the lat/long of a counting site, and the counts can be and were summed to include vehicles passing in either direction and in any lane.

The organization and motivation of the two data sets is as different as can be. These differences are summarized in Table 2.1.

TDAT is a database of project-specific traffic counts maintained by the City of Portland. TDAT is continuously being added to at publication time.

The database is large (885 MB of Structured Query Language (SQL)) and spans a lot of time (1986-2006). TDAT is largely undocumented outside of suggestive table and field names, and in a white binder in the corner of an office at the PBOT. We connected software for graphically representing the data dictionary, in order to create Figures A.8 and A.9.

We obtained a GIS layer from the regional authority which assigns one of 32 road classes to each segment of road in the metropolitan region. A map of part of this area is given in Figure 2.1. 


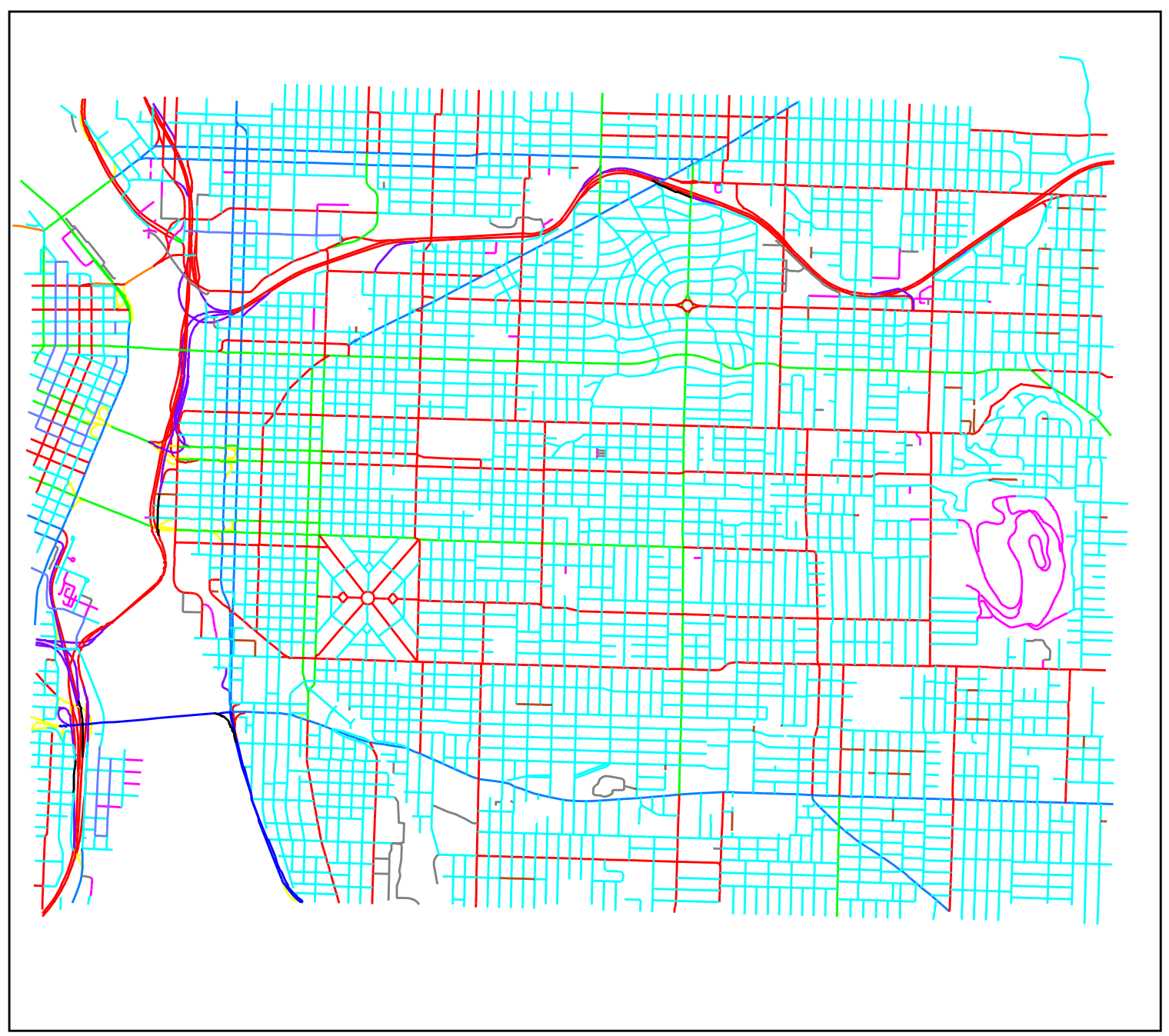

Figure 2.1: A map of a part of the study area showing colors indicating road class. The colors are as given in Table A.1. 


\section{Portland, OR, USA Traffic Count Sites}

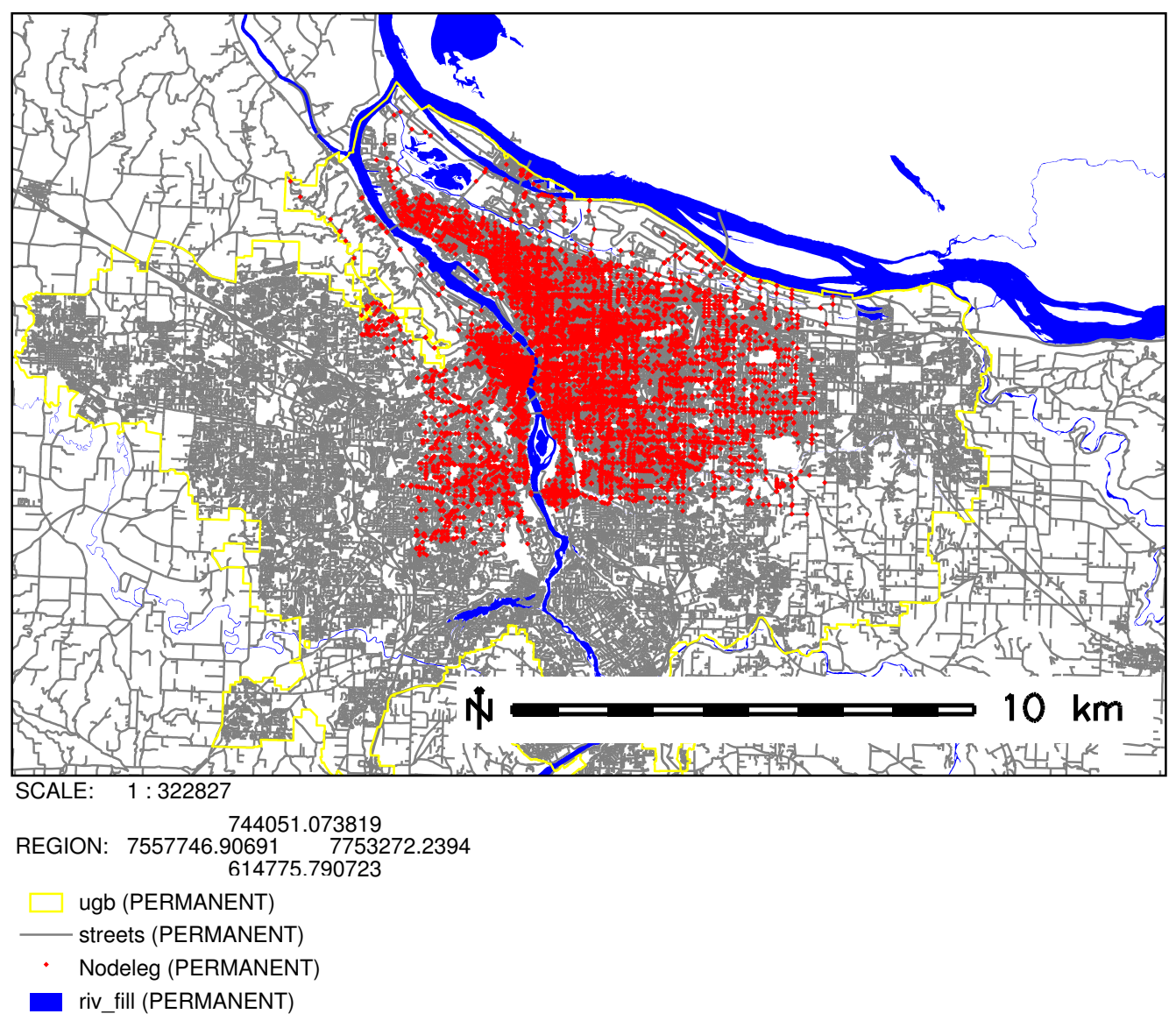

Modification date: Mon Jul 29 18:31:58 2013

Map created by James Powell 2013-07-29.

Source: Metro's RLIS archive and Portland, OR's TDAT2 traffic count database.

Figure 2.2: TDAT count sites in the Portland Metro Area. Red dots are sites. The yellow line is the Urban Growth Boundary. The blue is the Willamette and Columbia rivers, and the gray lines are streets. Data sources: Metro's RLIS GIS data and the PBOT TDAT2 archive. 


\subsection{DATA CLEANING AND REDUCTION}

To gain confidence in the usefulness of the TDAT data, we mapped the count sites. The result is in Figure 2.2. We observe that coverage is intense and fairly uniform in an area centered on downtown, but no coverage exists outside of the city limits leaving much of the metropolitan area unsampled.

PORTAL

PORTAL 40 is an archive of regional freeway and highway traffic counts kept by PSU. PORTAL is continuously being added to at publication time. The project combines the resources of PSU, Oregon Department of Transportation (ODOT), Portland Metro, and the US Department of Transportation. Vehicle counts gathered from a network of 732 loop detectors planted in restricted roads in the Portland area are recorded for research purposes. Recordings are made at 351 different stations.

To gain confidence in the usefulness of the PORTAL data, we mapped the count sites. The result is in Figure 2.3. We observe dense and uniform sites throughout the metropolitan region.

"ItâĂŹs like riding a psychotic horse toward a burning stable" (Robin Williams as Armand in "The Birdcage")

Our problem is similar to probing a crystal with a laser from many different directions to find paths with no defects. Defects that are found are categorized and the suspect data is cleaned out of the final product.

We resolved this problem by building an eight-stage pipeline, where each stage except the last cleans the data, identifying and isolating outliers. The last joins the clean count data to separately prepared "land use" data such as functional 


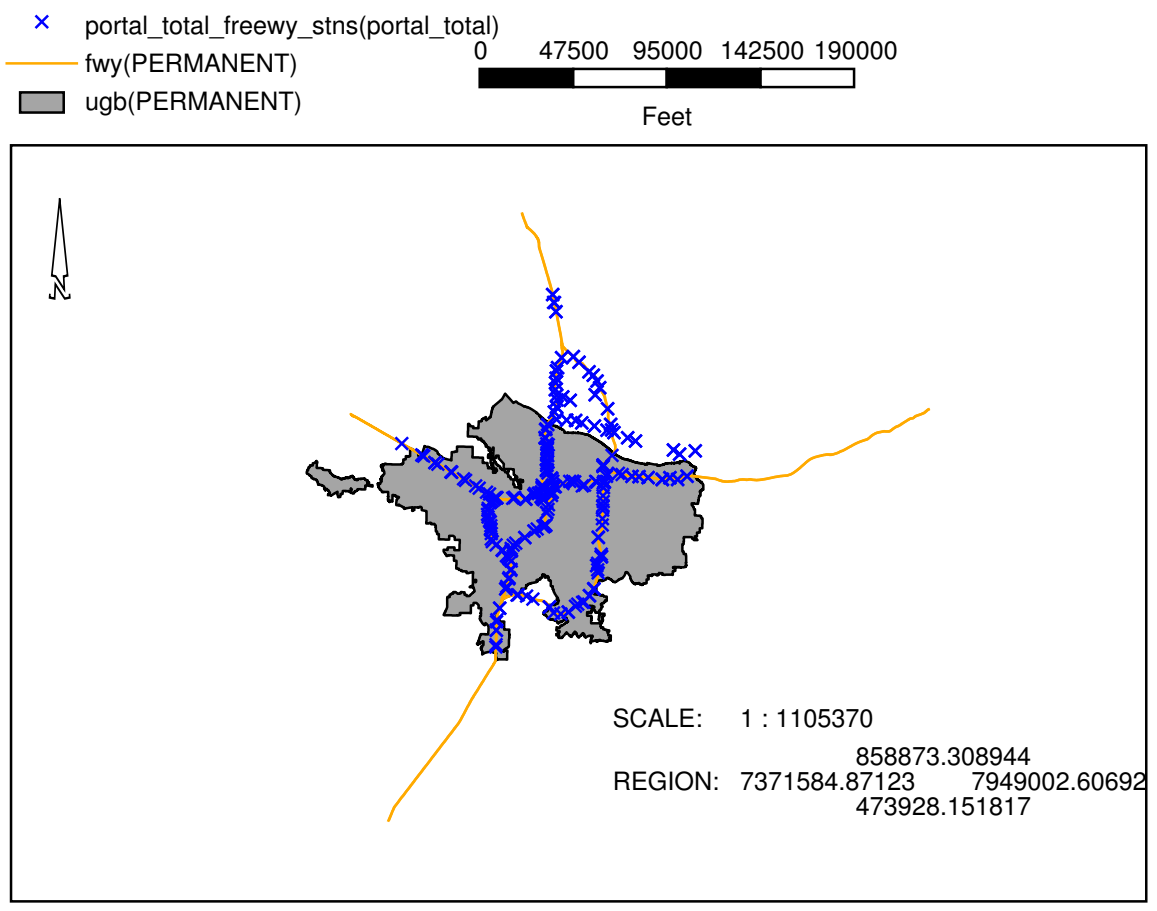

Map created by James Powell, 2014-10-20.

Data from RLIS (layers ugb, fwy) and PORTAL

(portal-total-version-2014-07-31/portalstations.csv).

Figure 2.3: PORTAL sites in the Portland Metro Area. Blue crosses are stations, yellow lines are freeways, and the gray area is the urban growth boundary. 
road class, population density, and on-ramp mileage in the area. The pipeline is presented in Appendix A.2.

Data Quality Problems Common to Both Archives

The majority of our data cleaning filter applies equally to TDAT and PORTAL. We used a computing mechanism called materialized views to cast PORTAL in the guise of TDAT to enable a single code base to operate on both databases. We will discuss the common problems now.

Negative Counts Both archives have a handful of instances where a count of less than zero was recorded. Discovery of this defect necessitated marking the whole counting day as an outlier.

Two (or more) channels of data exist that share only a single channel number. These channels (a TDAT term, these are known as detectors in PORTAL) are expected to be uniquely identified. Some counting days in both archives have multiple channels assigned to a single channel identifier.

Missing Data for a Channel We organize our counting into one or two channels. One channel counting indicates traffic flow in a one-way street (PORTAL highways and freeways are always two-channel). Two channels require traffic flow in two directions.

Both archives have a number of instances of data being recorded for one channel or lane of traffic, but the record for an expected second channel is missing entirely from the archive.

Incomplete Day Of Recordings The vast majority of outliers in both archives is the case where the recorder was only able to obtain part of the full day's recordings. 


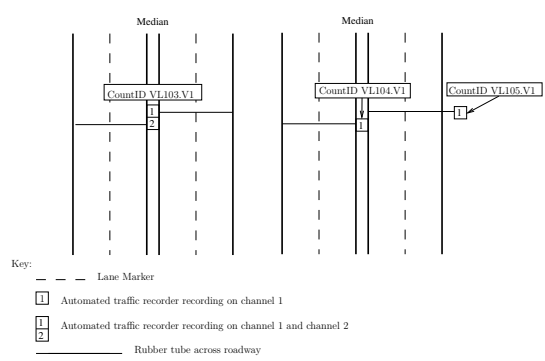

(a) Normal arrangements.

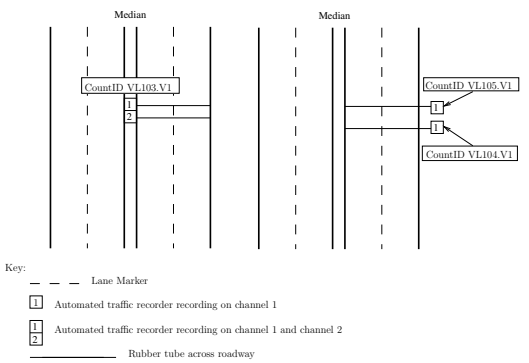

(b) Pathological arrangements.

Figure 2.4: Known TDAT counter arrangements. Numbers inside of square boxes are channels.

A typical example will have recordings starting at $11 \mathrm{AM}$, and ending at $2 \mathrm{PM}$.

The JAMAR-type traffic counters (pneumatic tubes and recorders manufactured by JAMAR corp.) used by PBOT have multiple channels (multiple tubes connected). The counting arrangements in Figure 2.4 capture all of the possibilities and are expected to be typical of project-level automated counting in any city. We can confidently report that TDAT's long history spanning 21 years and perhaps 8000 forty-eight hour projects has enabled ample exploration of the ways the data can be collected and recorded.

The Number Of Channels Is Not In $(1,2)$ For This Count A slight handful of TDAT counts were marked as outliers because the number of channels was outside our canonical range of 1-2.

Unacceptable (or NULL) Excepttype TDAT has a field excepttype which usually indicates normal weekday, or normal weekend. Any alternative, for example the code \#BIKE which indicates "count includes bicycles only, no motor vehicles" and the code \#CONST which indicates "obstruction count data affected by 
construction activity which does not close the entire roadway" 1 are excluded.

No Kounts Were Recorded Under This Count Id We introduce here the TDAT jargon of "kounts". A kount is an integer expressing the number of vehicles recorded as passing the device in a given time period, while a count is the introduction of a project-specific counting event. There are many cases in TDAT where a counting event was introduced (so a count ID was made) but no "kounts" were ever recorded.

PORTAL-specific Data Quality Problems

PORTAL displayed a number of the same DQ problems that we found in TDAT. Two unique problems with PORTAL are detailed here.

PORTAL Station With No Other In the case of roads with bidirectional traffic, we require simultaneous recording of traffic in both directions. Many PORTAL stations do not supply this comprehensive recording; counts at these stations are filtered out.

The Use of the Computer for Reproducibility and $\S 2.5$

Automation

The analysis is enabled by automation. To reproduce our work, one begins with a "tarball" we have on our research computer - a single file which any programmer can easily unpack and build. The build concludes with a single executable file containing machine code and embedded SQL code, R code, and GIS map specifications. This program is executed from a Portable Operating System Interface - Unix (POSIX) command line, and when it finishes (in about 5 days) the Data Preparation and Modeling phases are complete.

\footnotetext{
${ }^{1}$ Private communication from PBOT's J. Throckmorton, 2014-07-22
} 
As the research proceeds, we are configuring our computer to automatically repeat the above on a rolling basis, as a safeguard against the unfortunate case of a bug being introduced or fixed without notice and documentation, which changes our model in a meaningful way.

It is best practice to reuse software components when possible and to thus diminish the impact of a syndrome or anti-pattern known as "not invented here." We have maximized our use of other people's work, reducing our own work to a mere 10,000 lines of code. The relatively small footprint, which is only $4 \mathrm{x}$ bigger than the MAEROS coagulation model, for example, and just $1 \%$ of the size of the WRF research-grade meteorological model, is a great aid to us allowing transparency and parsimony to be the rule of the day. The software stack is shown in Figure 2.5. and most of these components are probably recognizable to the reader because of their famously useful rôle in a great number of computer-based experiments.

We were able to achieve this high level of automation and reproducibility by virtue of an initial, risky effort to build a program-that-programs, which we call the Zombie Caterpillar. The driver, called tache 2 at the top of the stack is able to initiate processes amongst any other component in the stack, locally or over a network, and interact with that component in complex ways. One example of the utility of the Zombie Caterpillar is that among the $10 \mathrm{~K}$ lines of driver code is an Application Programming Interface (API) of great generality and depth for carrying out GIS analyses. Another example is the ability of the driver to call out to $\mathrm{R}$ for calculating statistics and other data reduction and error analysis steps. We also use Embedded SQL 3 a type-safe and null-aware means to use the archive from $\mathrm{C}++$.

\footnotetext{
${ }^{2}$ Tache: a French word meaning task, but also stain, as in the stain of $\mathrm{CO}_{2}$ in our atmosphere.

${ }^{3}$ Embedded: the interpreted SQL is embedded into a compiled $\mathrm{C}++$ code.
} 


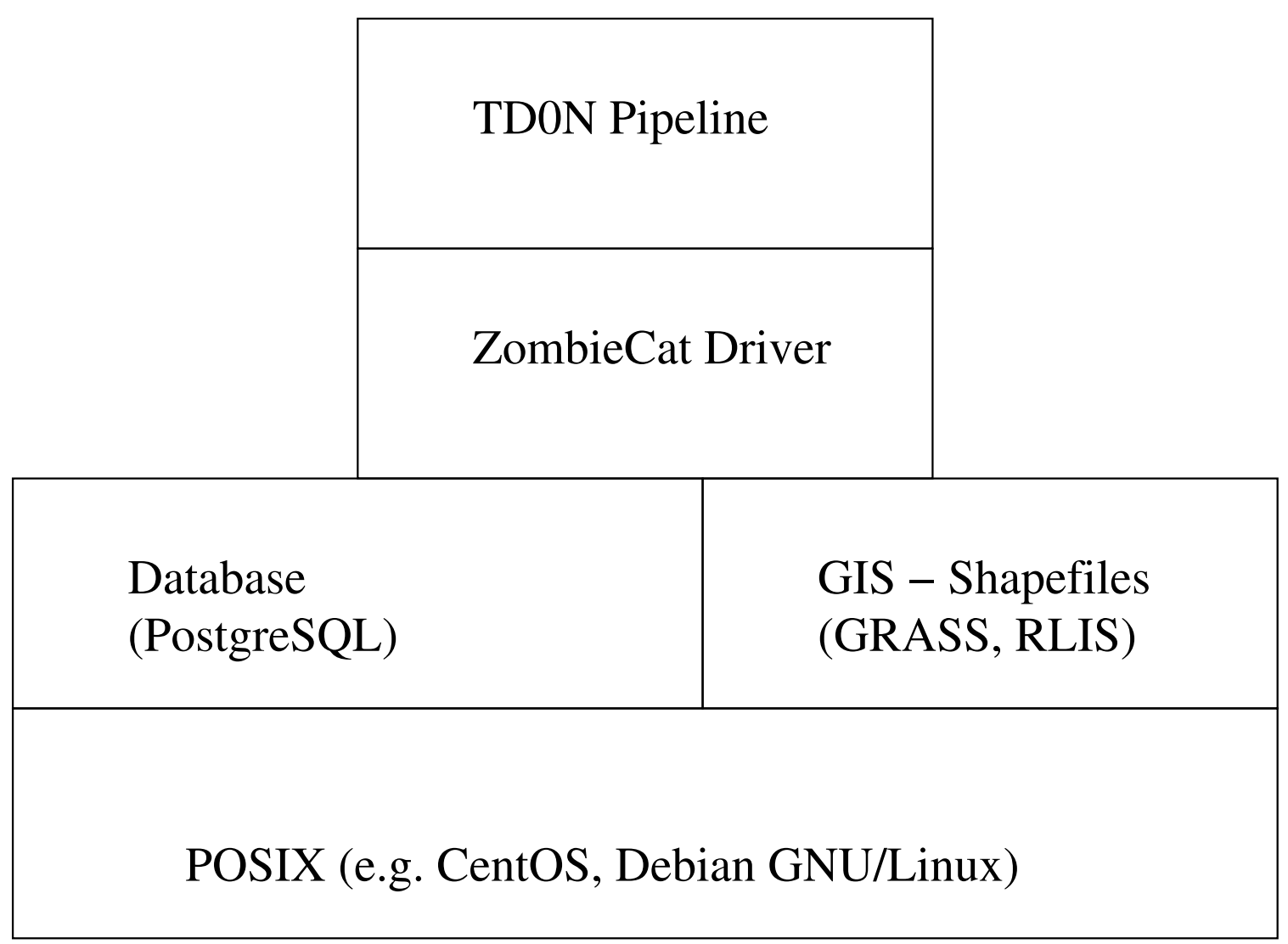

Figure 2.5: A schematic of the software stack used in the research. 
We carried out an extensive statistical analysis of the cleaned data after our cleaning process completed. These steps involve data reduction and error analysis [41, and stepwise multiple linear regression [38] [28]. Quantile-quantile plots [42] [43] were used as diagnostics to demonstrate the normality of the distribution of the $\epsilon$ of the traffic counts in our linear regression. The linear regression was analyzed by holding back one third of the data for validation, and also by graphing the data at various levels of detail and using different slices or subsets to produce results and to test theories including a putative large weekend effect and a smaller seasonal signal.

This concludes the overview of our methods. We will now describe our methods in greater detail.

To select our data set, we started with all available counts. We then began the process of accumulating a set of very clean 24-hour days of steady counting. We then assigned a road class to each count site (Appendix A.5).

These two types of data, typified by the tuple (number of counts, timestamp of the beginning of a fifteen minute interval, lat, long, road class), are the desired result of preparing our data sets.

TDAT data were prepared by obtaining SQL for the entire database and associated GIS layers supplied by PBOT. 
As described in Section 2.3.2, we prepared the PORTAL data by making a GIS layer of the count stations and obtaining a complete record of the raw counts using the PORTAL web site. We decided that the best approach would be to use SQL views to cast one of the data sets into the form of the other. Success here meant that a single data cleaning code could manage both archives with little special-casing.

This approach proved beneficial. We found it easy to put PORTAL into the form of TDAT using Cartesian joins (a process where by data with packed coding, meaning little redundancy, is exploded to produce a simple, flat table with a lot of redundancy), and since the data are so similar in essence although very different in organization this step represented a classical unification of apparently disparate systems. It is very simply expressed, and it makes our system robust to accepting data in either form, while both forms are apparently completely logical in the context of the Bureaus that record the data.

The SQL code for this adapter is given in Appendix A.6.2.

We require a continuous source of $\mathrm{CO}_{2}$ for our program of eventually cross checking our model against measured $\mathrm{CO}_{2}$ concentrations, but we're given discrete (in both time and space) examples in which the passage of a vehicle is noted at a particular location and time. We need to fill in spatial and temporal gaps in the discrete data to give reasonable values for traffic counts where no count data exist.

The map in Figure 2.6 demonstrates both the problem and our solution for the spatial part of the problem. The points marked $\mathrm{X}$ are the discrete historical cases where vehicles definitely were observed passing by during the time period of interest. The color of the lines is given by our model. We desire a close agreement 
between these values.

The continuous values along the road segments are the product of a linear regression. We asked: what factors drive traffic volumes? As we see in 44, a number of factors are possible: population density, greenness indices, distance from the center of the city, even the vertical slope of a road segment. We generated the list of Table 2.2 and selected just population density and meters of restricted road onramp-type road in circles of $100 \mathrm{~m}, 200 \mathrm{~m}$ etc. out to about $800 \mathrm{~m}$.

We will now derive an equation of the form $A D T=a_{1} R C_{1}+a_{2} R C_{2}+\cdots a_{n} P O P D E N+$ $a_{n+1} O N R A M P$, and describe a process for filling the gaps in the emissions inventory, gaps in between count sites. The analysis proceeds by augmenting a matrix whose initial contents are only the lat/long of the count sites, 6000 of them, so it's a 2x6000 matrix which for reasons which shall become clear, we call nodeleg_866_vector_repo_001. We will augment this with road class, population density, and on-ramp measurements.

Establishing the Log-normality of the Counts

"In general, we must keep in mind that there is no guarantee that use of these transformations will necessarily be better than analyzing the proportions directly; much depends on the data. The effectiveness of a transformation is best assessed by trying it on the data and then checking the fit of the model and the pattern of residuals that results." [28, p. 239] on using log- or arc-sine transformations on proportional data.

"In the standard classifications, skewness in the distribution of errors 
High resolution traffic density model for Portland, OR

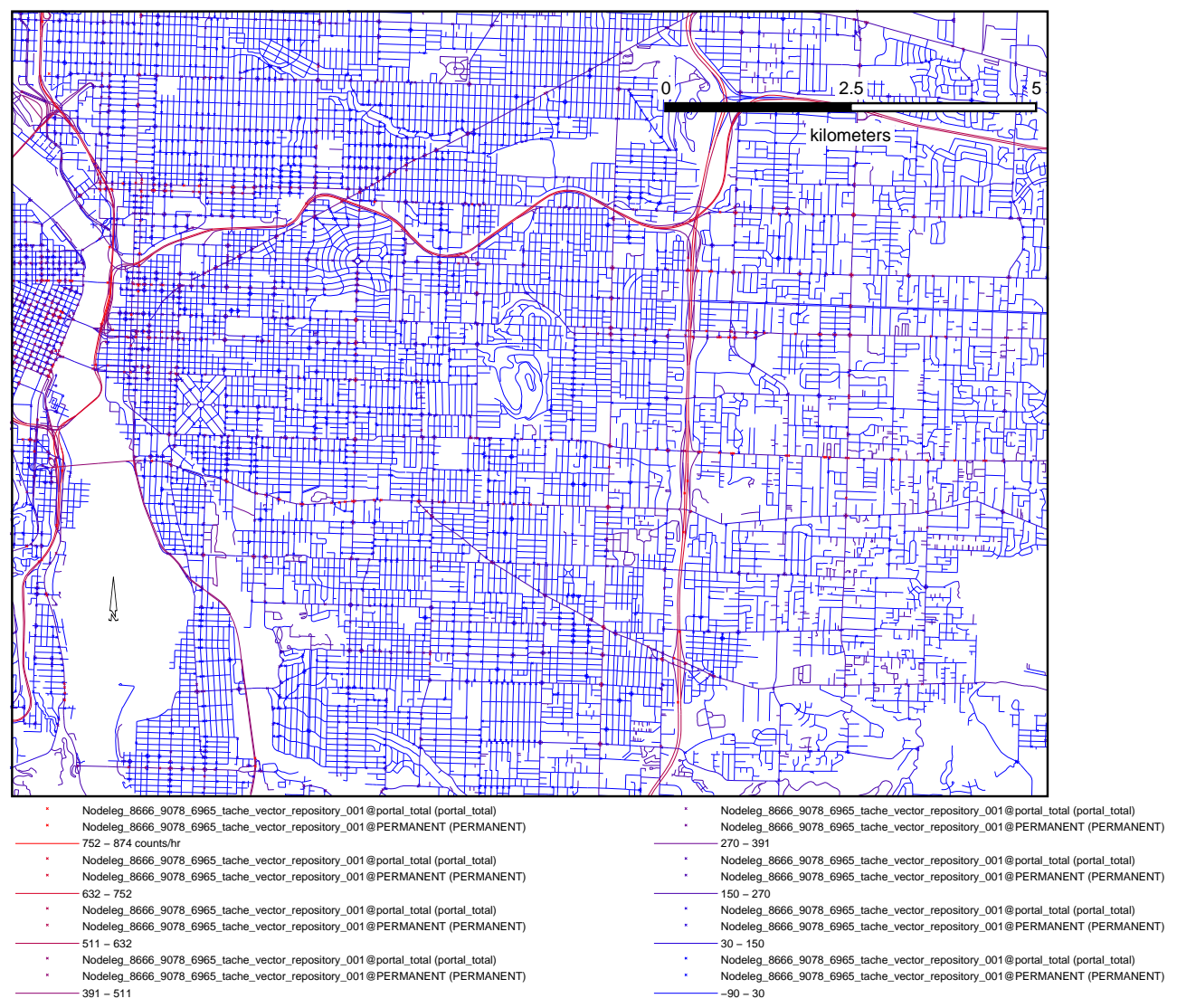

Map created by J. Powell, 2014-12-12.
Data sources: RLIS streets, PBOT TDAT, PORTAL, TIGER census 2010.

Figure 2.6: Actual counts and model counts on a map of Portland, OR. Vertices are raw data, while the color of the line segments is modeled based on road class, population density, and on-ramp mileage. 


\begin{tabular}{lr}
\hline Variable & $\begin{array}{r}\text { Subjective } \\
\text { Importance } \\
\text { (out of 10) }\end{array}$ \\
\hline Road class & 10 \\
population density & 10 \\
meters of hwy on-ramps & 10 \\
percent of land cover that is & 5 \\
impermeable (or tree covered, or lawn) & 0 \\
slope & 0 \\
aspect & 0 \\
proximity to (school, hospital, groc. store, etc.) & 0 \\
zoning mix & 0 \\
could train neural net on all poss. variables - \\
soln is then general & \\
temperature, precip, humidity & \\
day of week & \\
weekend or not & \\
holiday or not & \\
month & \\
quarter & \\
season & \\
year & \\
decade & \\
major sporting event or concert that day & \\
Obama visits & \\
crime rate & \\
direction of travel (EW vs. NS) & \\
proximity to river & \\
CO2 intensity recorded by Dr. Rice at 3 area locations. & \\
Other Air Quality measurements by L. George & \\
Traffic as counted by other counting systems & \\
(L. Hill's thesis) & \\
New metric: is it on a line of steep travel gradient & \\
Distance to Max, Bus (limit the years & \\
in which red/yellow Max line operated & \\
L. George's student who has time series of NOx in PDX & \\
Volume-weighted Road Density & \\
\hline
\end{tabular}

Table 2.2: The result of our brainstorming effort, informed by research, about factors that could sensibly affect traffic counts in the city. 
tends to produce too many significant results in $\mathrm{F}$ - and t-tests. In addition, there is a loss of efficiency in the analysis." [45, p. 325]

We began our research expecting to find that our data would follow a lognormal distribution, because of the proportional nature of the data. Log-normality is expected if the treatments have an effect that is proportional instead of additive: an increase of $5 \%$ per unit of treatment instead of an increase of 5 units 45 , p. 329]. We consider it reasonable that for a given site, the evidence of the population density or mileage of on-ramps in the area around the site does suggest a proportional increase or decrease of the ADT compared to the mean ADT for that road class. This makes a log-normal distribution of the traffic counts likely.

Our exploration supports this assumption. Graphing the data is the best way to observe log-normality, and we see evidence of log-normality in the histograms of Figure 2.7, in which we plot the raw data. The result is obviously not Gaussian, or the histogram would look bell-shaped and the Q-Q plot would approximate a straight line. The following Figure 2.8 displays the data after a log transform. The Gaussian bell curve is apparent.

Homoscedastity, which is the property of constancy of the variance of the error term over the full range of the model fitted to the data, is an assumption of Ordinary Least Squares (OLS) fitting (we include a complete list of OLS assumptions in Section 3.0.11. It is only by homoscedastity that OLS is the "minimum variance unbiased estimator" 4 . In the absence of homoscedastity, the estimator has more variance, but it does not pick up a bias (ibid).

When the data are log-normal (that is, the logarithm of the raw data are normally distributed and the raw data themselves have the greatest number of counts at the low end of the distribution and exponentially decreasing frequency of counts

\footnotetext{
${ }^{4}$ https://stats.stackexchange.com/questions/52089/ what-does-having-constant-variance-in-a-linearregression-model-mean/52107\#52107 accessed 2016-10-23.
} 

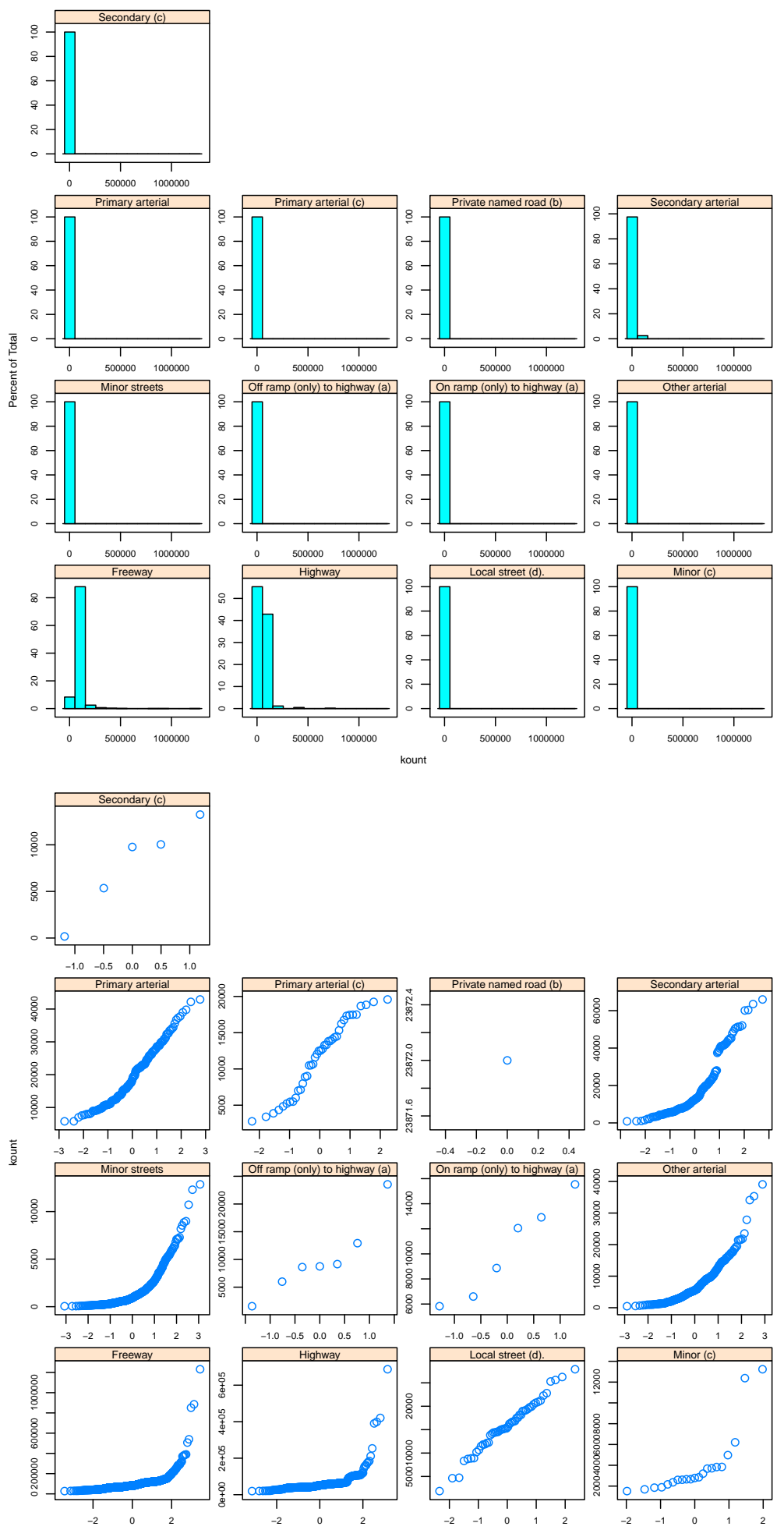

Figure 2.7: Histograms (top) and QQ plots (bottom) for a random sample of weekday ADT, untransformed. No indication of normality is seen in these graphs. Each panel is one of the road classes. Codes (a), (b) etc. are aszin Figure 3.5. 

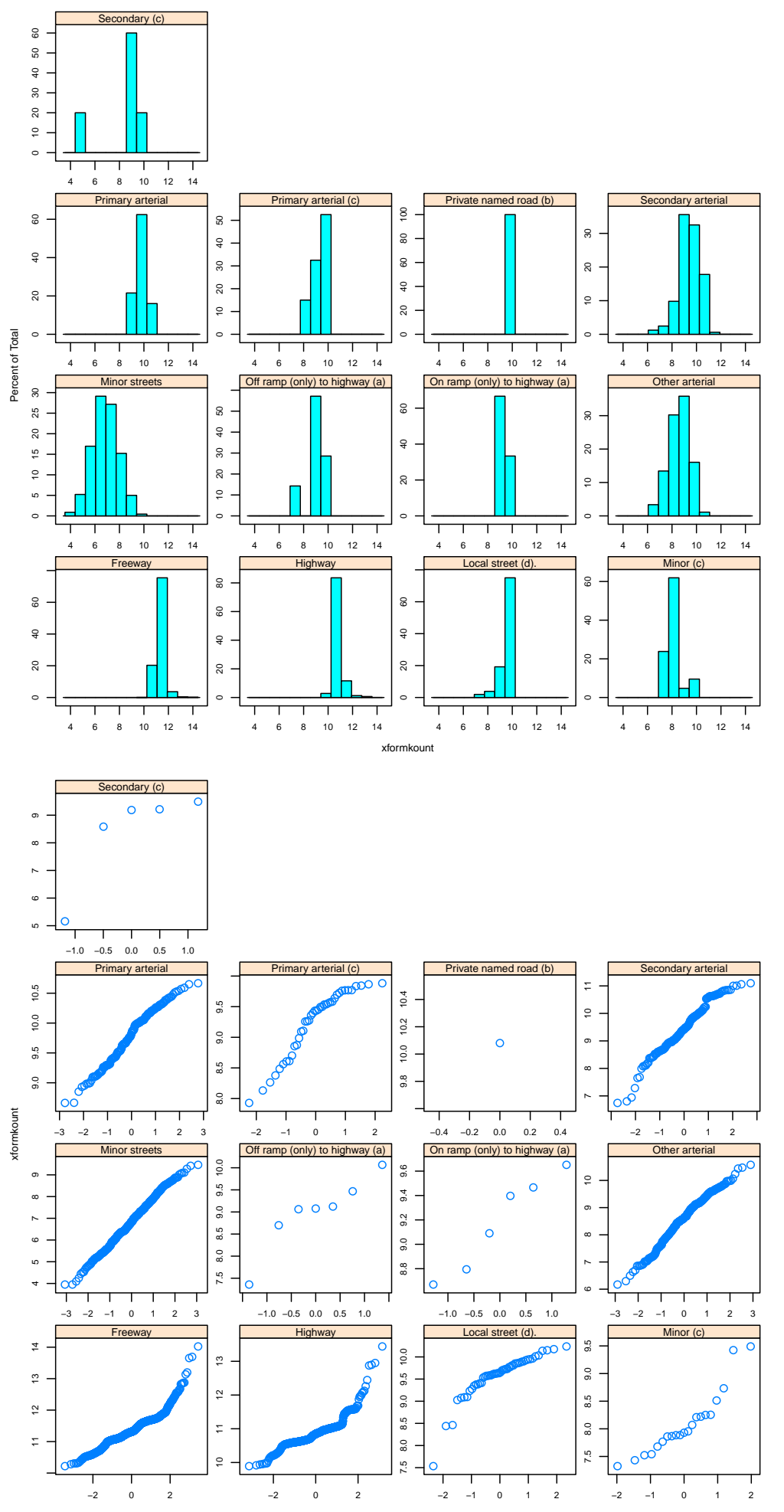

Figure 2.8: Histograms (top) and QQ plots (bottom) for a random sample of weekday ADT, log-transformed. Normality is seen in these graphs. Each panel is one of the road classes. Codes (a), (b) etc. are as in 2 gigure 3.5 . 


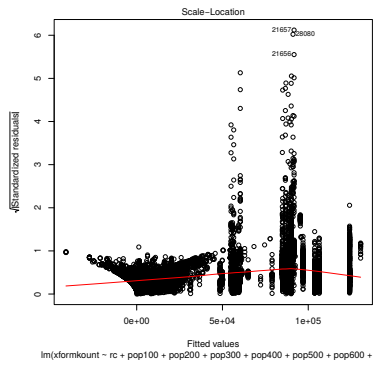

(a) Raw data.

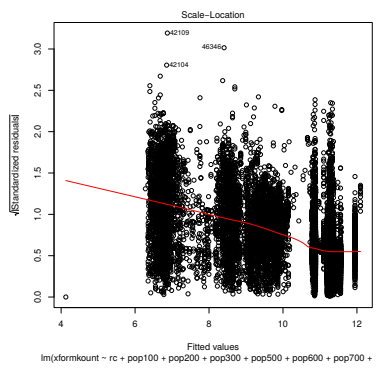

(b) Log-xformed.

Figure 2.9: In (a), the square root of standardized residuals plotted against the fitted values for all of the raw data, without a log-transform. Observe the definite proportionality of the variance of the residuals against the fitted values. In (b), after the log-transform the residuals appear more normally distributed around zero.

in the higher ranks), the variance of the residuals will become a linear function of the count 45, p. 329]. Figure 2.9 displays a proportionality of the variance of the residuals that is clearly linear over some parts of the data.

Additionally, the graphical evidence is clearly indicative of a problem with the constancy of the variance until the log-transform, after which the variance is highly constant.

We suspected log-normality in the count data because count data (missing symmetry around the origin) do typically follow a log-normal distribution. We plotted the raw data as a histogram and we examined the residuals using a quantile-quantile plot to look for signs of the lack of normality. Normally distributed residuals are one requirement for a valid linear model.

We plotted as a histogram the untransformed and log-transformed ADT for all road classes, and tested the arterial and freeway counts for normality using Shapiro-Wilk (shapiro.test in R, [46, p. 382]). We tested for normality using the same test again after a log transform. The code for these plots and tests is in Appendix A.6.4. 
We obtained an ADT record of 1.1 million count/days. We used our ADT data as input to a multiple linear regression following well established practice. The raw data were sectioned randomly using a high quality random number generator (R's sample) with $\frac{2}{3}$ of the rows marked "use for model" and the remaining $\frac{1}{3}$ of the rows marked "use for validation" using the code in Appendix A.6.9.

The raw data were log-transformed, a procedure justified by the log-normal structure of the data.

A stepwise algorithm, in particular step() in R, which uses the AIC metric (a metric which balances significant of each term in the regression against simplicity gained by having fewer terms) to decide on the importance of keeping a term, which resulted in a regression with 26 terms and an $r^{2}$ of 0.89 . Without stepwise, the regression has 28 terms. The terms for pop400 and pop800 were dropped during the regression as not contributing sufficiently to the AIC metric. The $\mathrm{r}^{2}$ is not affected by these two terms being dropped.

The results are presented in Section 3.0 .13 .

Obtaining VMT

We followed the technique of [18], in which VMT is calculated by multiplying ADT by the length of a stretch of road.

The Multiple Linear Regression

The software used in the linear regression is described in [46, pp. 403-414] and in [47, pp. 100-114]. This is research-grade linear regression applied in the most standard idiomatic manner.

The traffic counts emitted by the TD0N pipeline of Figure A.1 were subjected 


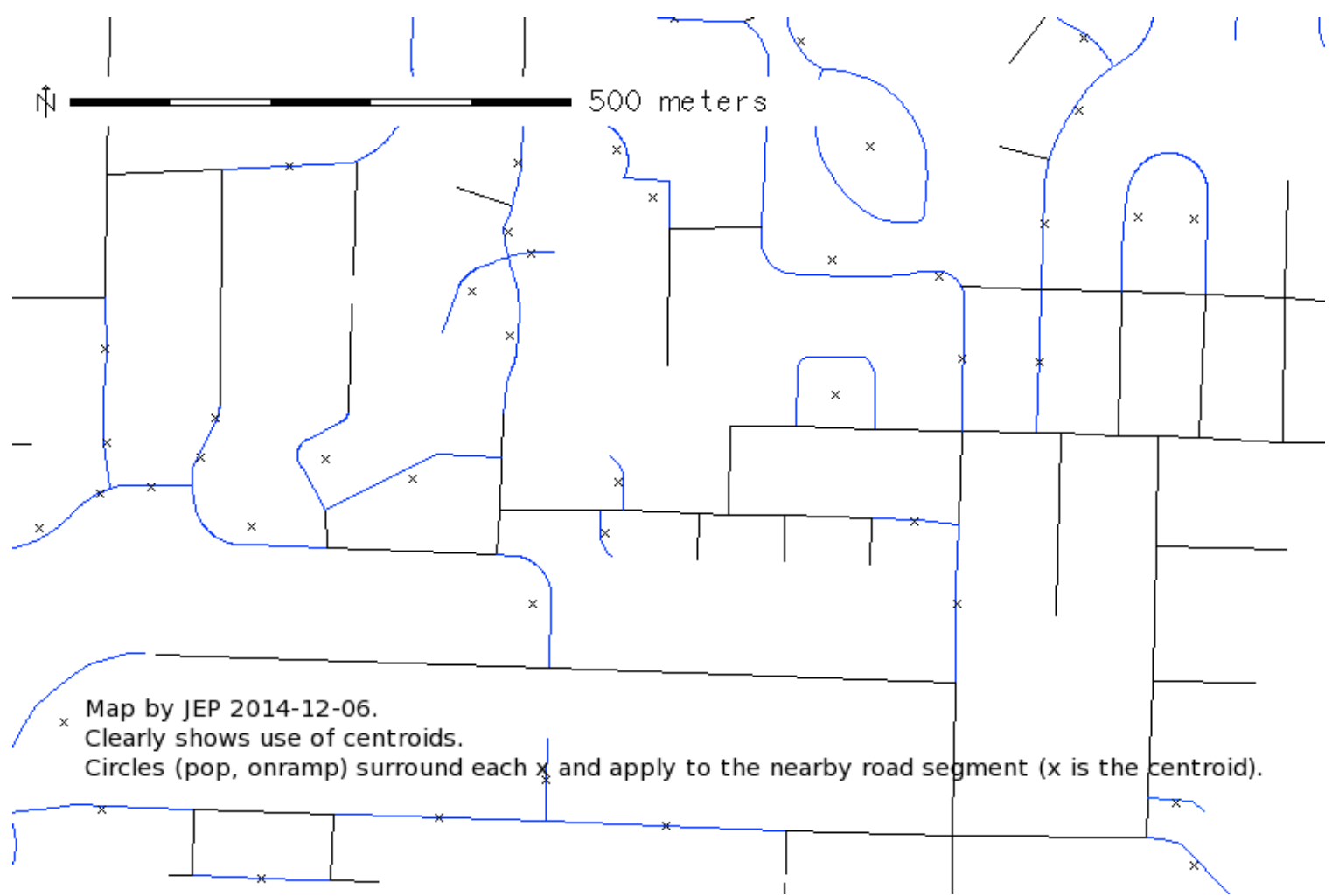

Figure 2.10: Centroids assigned to each road segment in RLIS streets.

to a battery of tests in an attempt to detect irregularities. We conducted this work as a series of experiments.

To obtain the pseudo-land use variables of population density and on-ramp density for each road segment in the city, we first needed a representative point for each road segment. Ideally this would be a point halfway along the road segment, but the technique we landed on gave us centroids for the geometry of the line segment. These centroids are indicated in Figure 2.10. 
We developed a layer of $\mathrm{N}=10^{5}$ centroids for road segments in order to use the linear model TD0N. For each centroid we evaluated several Land Use Regression (LUR), specifically population density from the 2010 US Official Census and onramp density ( $\mathrm{km}$ of onramp per $\mathrm{km}^{2}$ ). We evaluated these inside circles centered at the centroids with several radii: 100 to $900 \mathrm{~m}$ for population density, and 100 to $500 \mathrm{~m}$ for onramp miles, with $100 \mathrm{~m}$ increments. The calculation was estimated to require 28 days for completion. To shorten it, we developed a technique for using 250 central processing units (CPUs) on our Gaia computing cluster. This work required first learning how to deploy the GIS on those 250 CPUs. Once the GIS was operational however, this embarrassingly parallel computation completed in two days.

Population density was acquired from the US Census, 2010. The map of Figure 2.11 was created from the raw census data.

We constructed and used a few tools for data exploration. Our first look at the data graphically is the box plot of Figure 2.12 on page 35

We formed a series of logical hypothesis that we knew could be tested using the traffic count database. 


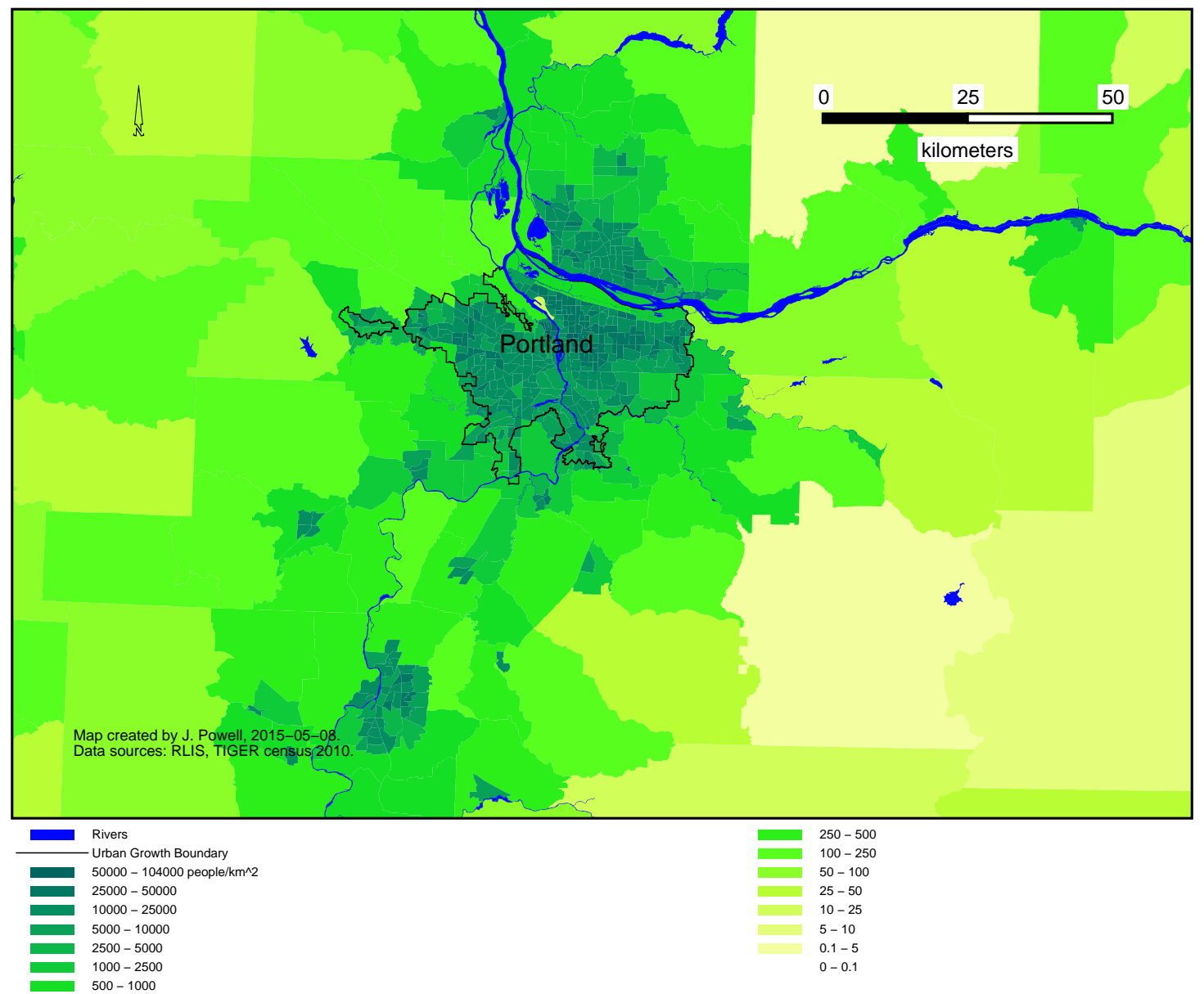

Figure 2.11: A population density map of the Portland, OR region. The rivers are a composite of RLIS's fine Metro-area base layer and TIGER's coarser state-wide coverage of waterways. The data are compiled at the level of the census tract, which is approximately the scale of a neighborhood. 


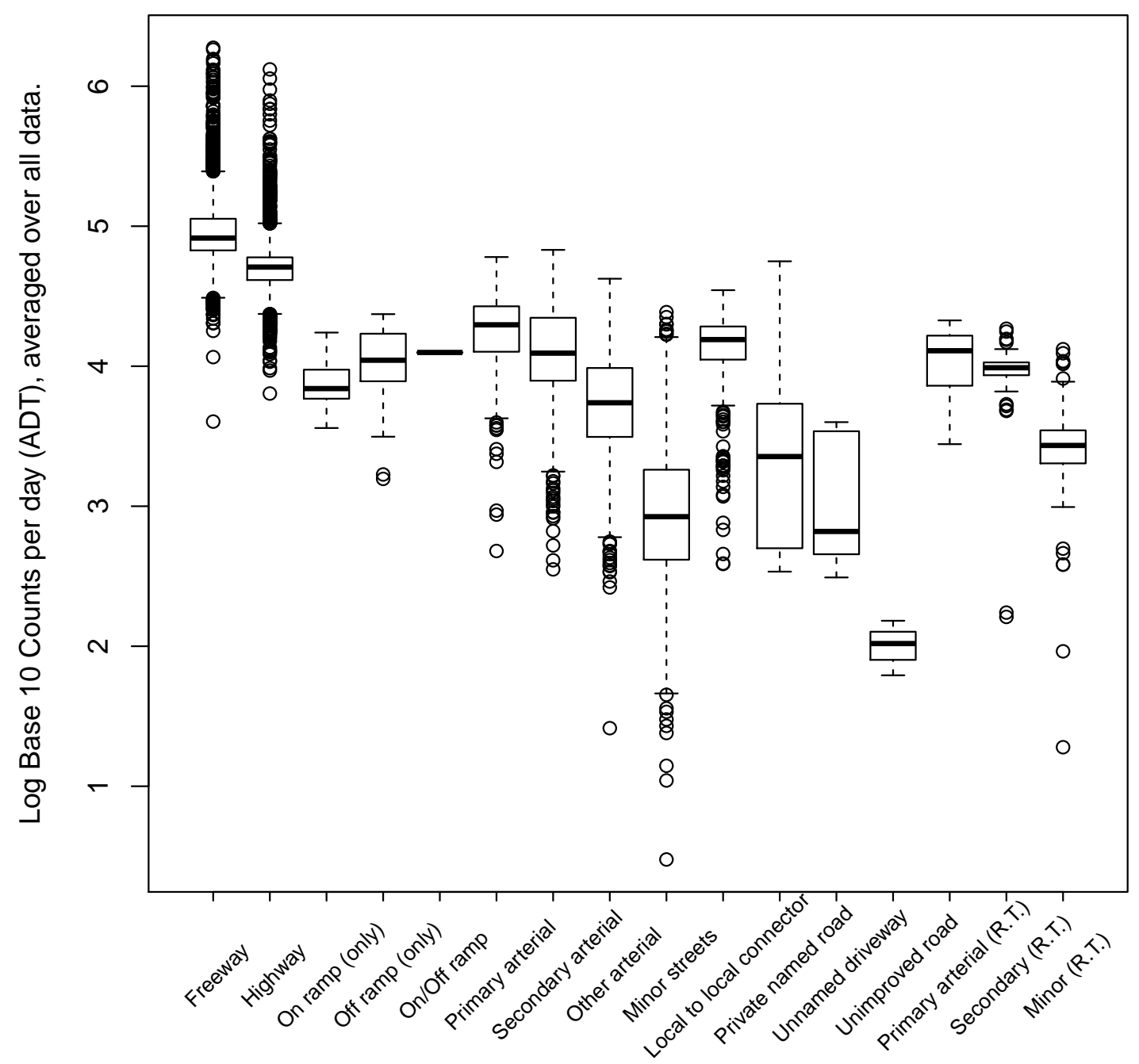

Figure 2.12: A box plot of the combined PORTAL and TDAT traffic count data after cleaning. $\mathrm{N}=34,308$ ADT values were used, each representing one day of counting at one site. The boxes represent the interquartile range: $25 \%$ of the data lie below the bottom line and $25 \%$ lie above the top line, while the interior line shows the median. Adjacent values are represented by "whiskers" (dashed lines) extending off of the top and the bottom of the box. Calculation of the adjacent values is described in [46, p. 242]; they show the approximate range of the data. Extreme values outside this range are drawn as circles. 
"The one-way analysis of variance model is one of the most useful models in the field of statistics. Many experimental situations are simply special cases of this model. Other models that appear to be much more complicated can often be considered as one-way models." [48, p. 2]

We believe that some of the terms we shall attempt to regress against will have a significant non-zero slope.

"Another way to view a distribution is the quantile-quantile (Q-Q) plot." 46. p.241]

It is routine nowadays to examine the quantile-quantile plot of a linear fit's residuals [43 47, p. 106]. We constructed a toy linear regression using values from a published table of Gaussian deviates [49] to practice linear regression and diagnostics. This code is present in Appendix A.6.1.

We used the same technique to produce diagnostic quantile-quantile plots for the real, entire data set.

Weekend Effect

Common sense suggests that traffic will be strongly attenuated on the weekend. This effect was clearly seen in a study of traffic in a 2012 study of Lebanon [50]. Closer to home, work by Kendrick and George in 2015 51, show this weekend effect.

To test for the presence of a weekend effect, we use the methods described in [39]: a t-test for the hypothesis that there is no difference between weekend and weekday mean values. 
Kendrick and George in 2015 [51], show the expected bimodal distribution on one of Portland's arterials. We show the same bimodal distribution with a morning rush hour and an evening rush hour. Another study in Lebanon showed the rush hour bimodality [50] which we reproduce for Portland.

Seasonal Effect

We investigated the possibility of a seasonal effect on the amount of on-road traffic. Waked et. al. saw more traffic in winter and less in late summer/early fall in Lebanon [50].

In collaboration with Dr. Andrew Rice, a data set of elemental analyses on moss was obtained. Errors in the latitude and longitude of the count sites were corrected and the sites were the moss samples were taken were mapped in Figure 2.13 . We attempted to find a correlation between high (low) traffic counts and high (low) nitrogen concentrations in the moss. 

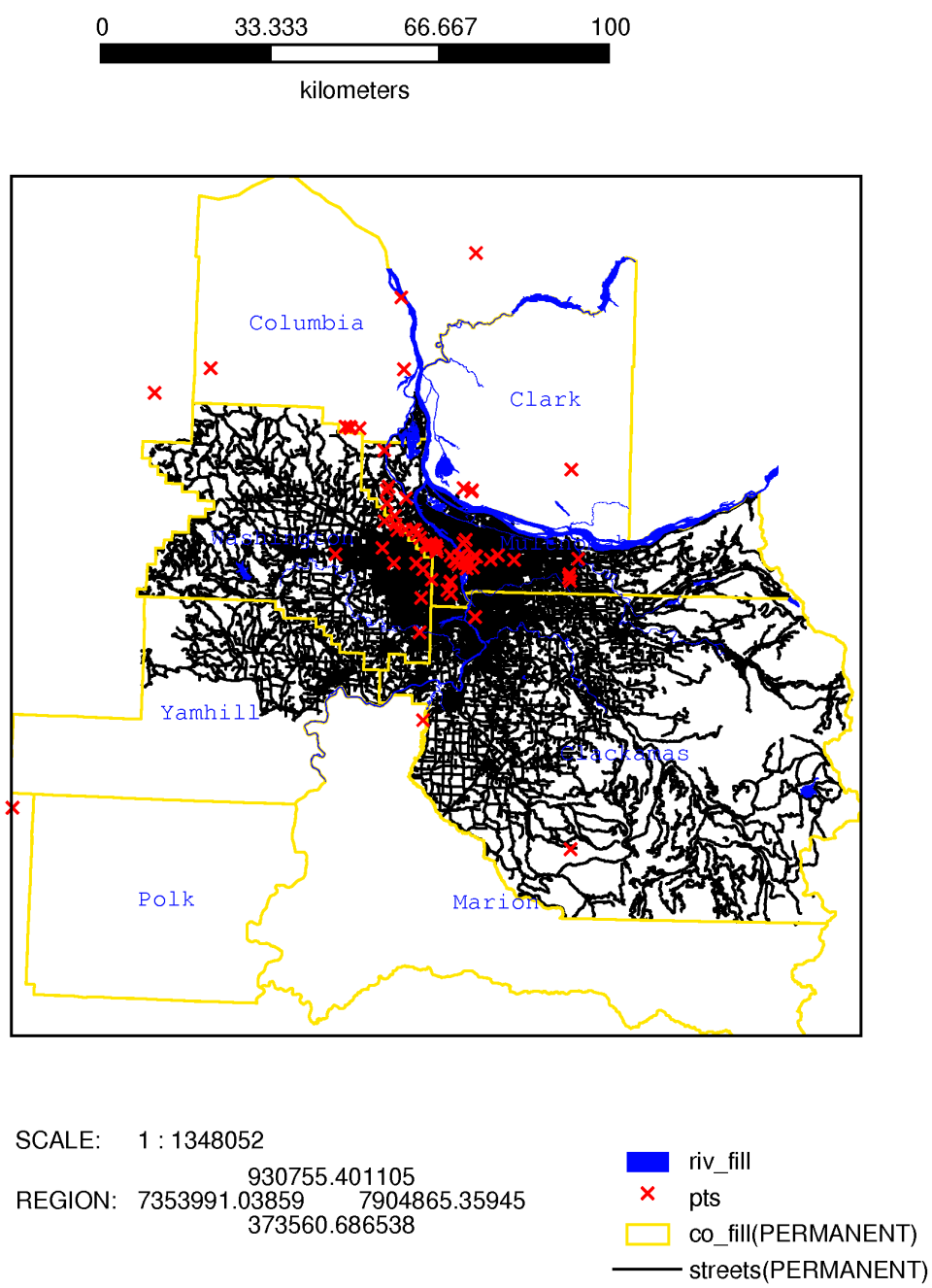

Map by James Powell 2014-01-30. Source: Metro RLIS. Moss collection data from Dr. A. Rice

Figure 2.13: A map showing all 81 of the moss experiment sample sites. Red crosses are sample sites. Counties are named. 
Chapter 3

Results/Discussion

'A basic problem in analyzing a time series is to study the pattern of the correlation between values at different time instants, and to try to construct statistical models which "explain" the correlation structure of the series.' ([52, p. 2])

Our analysis proceeds by a series of hypothesis, tabulated in Table 3.1 .

Our analysis begins by analyzing the combined PORTAL and TDAT product. We will note and analyze the sets separately when any qualitative difference emerges.

In data cleaning, tables of locations and in some cases location+days were made documenting problematic data. Any data that did not seem to be part of a a completely normal 24 hour count was discarded. Approximately $48 \%$ of the data was not used to build the linear regression due to these irregularities, which are cataloged in Table 3.2 and Table 3.3 . The code producing these numbers is given in Appendix A.6.11.

For PORTAL, the results are given in Table 3.3 . 
ID Description

H01 Our ADT values compare well with other published estimates.

H02 High (low) moss nitrogen concentration correlates with high (low) traffic counts.

H03 The Shaprio-Wilk test will confirm that the count data are log-normal.

H04 Linear regression is appropriate for the traffic count data.

H05 The residuals of the linear regression are homoscedastic.

H06 The linear regression is statistically significant.

H07 That a linear regression will predict ADT in $1 / 3$ of

the data when the remaining $2 / 3$ is used to make the model.

The regression is based on road class and "land use regression variables" (population density and on-ramp mileage inside circles of various radii around the count site).

H08 There are few outliers in the cleaned data.

H09 Rush hours will produce a bimodal trend in the diel, and weekends will be highly attenuated in counts.

H10 Small seasonal differences are expected.

Table 3.1: A table summarizing the hypotheses tested in this research.

id description

location/

days affected

(approximate)

306 the number of channels is not in $(1,2)$ for this count. A count represents a case where a device was recording. These are identified by unique count IDs.

311 Negative kounts (less than zero) were recorded.

A kount is an integer expressing the number of vehicles recorded as passing the device in a given time period.

310 missing data for a channel

304 two (or more) channels of data exist that 1188 share only a single channel number.

301 Unacceptable (or NULL) excepttype. We expect to find e.g. "Normal Weekday".

ExceptType codes interesting facts about the count day, such as that it was on a weekend, or a holiday, or any ad hoc note such as "Lane Count" or "Bike Lane".

298 no kounts were recorded under this count id 9170

303 incomplete day of recordings $\quad 56168$

Table 3.2: The types of outliers located in TDAT with an approximate number of location/days affected. The id column is a unique identifier assigned to the outlier type. 
id description

location/

days affected

(approximate)

296 PORTAL station with no other - it sits on one side unknown of the freeway with no station on the other side.

309 Negative kounts (less than zero) recorded $\quad 7$

A kount is an integer expressing the number of vehicles recorded as passing the device in a given time period.

305 two (or more) channels of data exist that 588 share only a single channel number.

307 missing data for a channel 2328

308 PORTAL count w/midday zero kounts 3110 (hr between 5 and 20)

302 incomplete day of recordings 12987

Table 3.3: The types of outliers found in PORTAL with an approximate number of location/days affected. The id column is a unique identifier assigned to the outlier type.

H01: Our ADT values compare well with other published $\S 3.0 .8$

estimates.

As one example, 51 reports "SE Powell Boulevard [in Portland, OR USA] is a major arterial roadway that runs east/west with peak hourly traffic volumes of 2800 vehicles and 28,000 Average Annual Daily Traffic (AADT)”.

The Regional Land Information System (RLIS) streets shapefile identifies SE Powell Boulevard as having road class 1300 (primary arterial). Our data shows that for this road class generally the weekday AADT as being 20335.

We found that of all of the sites recorded in PORTAL, the ADT over the nine year period sampled is 83808 vehicles/day. This is within the range of values reported for these same roads in the the "Atlas of Oregon" [53], which gives a number of 73,700 vehicles per day passing on the I-5 freeway in the year 1998.

Our work to build an EI to compare with other published estimates indirectly validates our ADT model (see Section 3.0.19). 
Results/Discussion

H02: High (low) moss nitrogen concentration correlates $\S 3.0 .9$ with high (low) traffic counts.

We evaluated TD0N at sites where plant moss samples had been collected and fed into an elemental analyzer, and searched for a correlation between TD0N and [N] . demonstrating a wider and possibly more important application of the model in investigating transport-related air quality issues. We used the same method as 54 , which [11] calls "volume weighted road density index" to predict $\mathrm{N}$ for moss.

We were not able to find evidence for this hypothesis. This experiment used an early version of our regression model which did not include freeway information. We were also unable to complete the experiment at 21 of 86 moss collection sites because they're outside of the Metro region and we have been unable to obtain street maps with the requisite road class information for this region. The map in Figure 3.1 shows these 21 sites. Our numerical analysis produced the graph in Figure 3.2 . The best fit line has a surprising negative slope, which is statistically significant $(p<0.001)$. We believe that either or both of the limitations of the experiment (lack of freeway, lack of sites in remote regions), both of which reduce the available experimental sites to a highly homogeneous collection of inner-city non-freeway variety, make detecting this signal - which is obvious in other maps of the nitrogen concentration - impossible.

H03: The Shaprio-Wilk test will confirm that the count $\S 3.0 .10$ data are log-normal.

We used the Shapiro-Wilk test to confirm our suspicion that the count data are log-normal, but the results indicate that the distribution of ADT for the freeway and arterial data are still unlikely to be normally distributed $\left(p<2.2 \times 10^{-16}\right)$. 
Feet

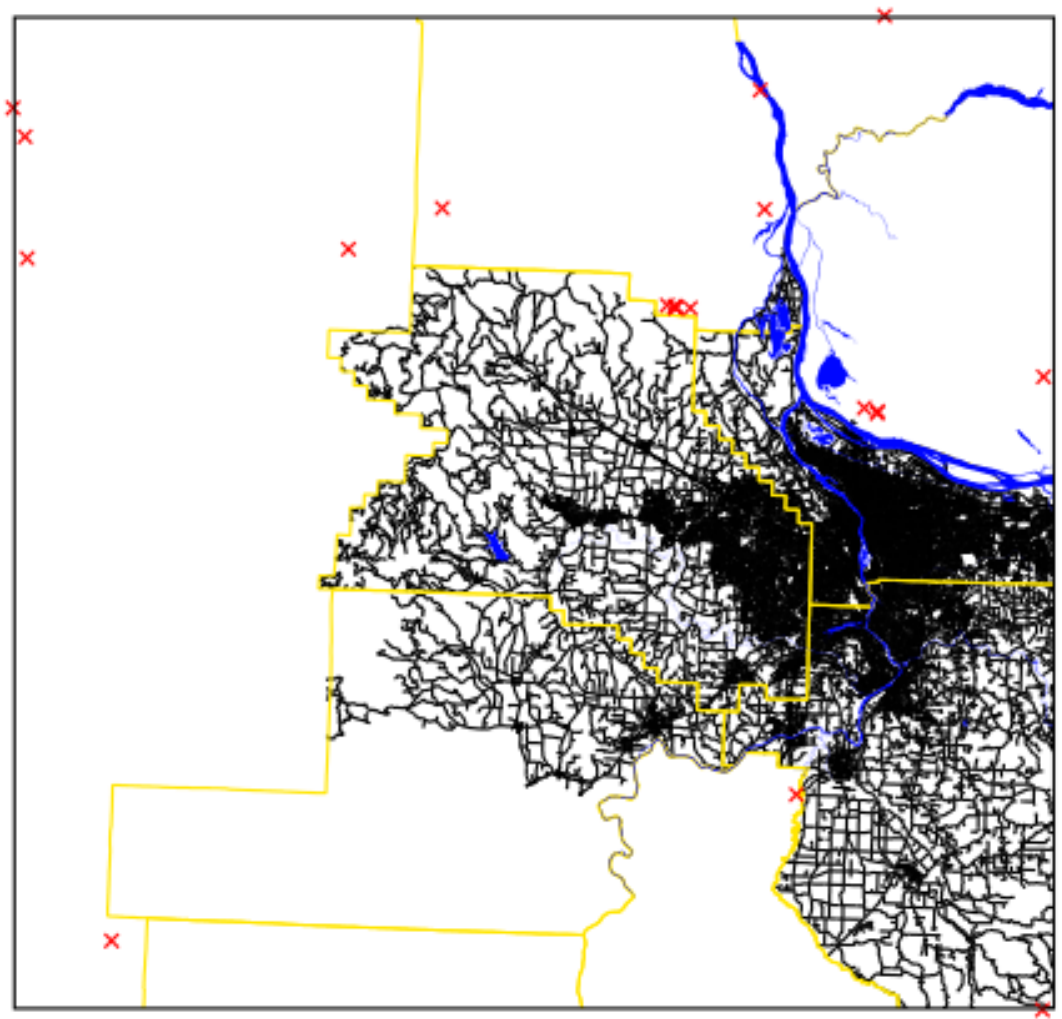

SCALE: $\quad 1: 932381$

880275.25

REGION: $7317285.19 \quad 7721317.13$

494949.78

aiv_fill

$\times$ pts

Co_fill(PERMANENT)

streets(PERMANENT)

Map by James Powell 2014-01-17. Source: Metro

RLIS. Moss collection data from Dr. A. Rice

Points marked in red are locations where moss samples

were taken that also have the characteristic of zero

road class information in Metro RLIS in a circle $200 \mathrm{~m}$ in radius centered on the point.

Figure 3.1: Moss collection sites outside of our ADT study area. 
Nitrogen vs. Traffic Density in a $200 \mathrm{~m}$ Radius Circle

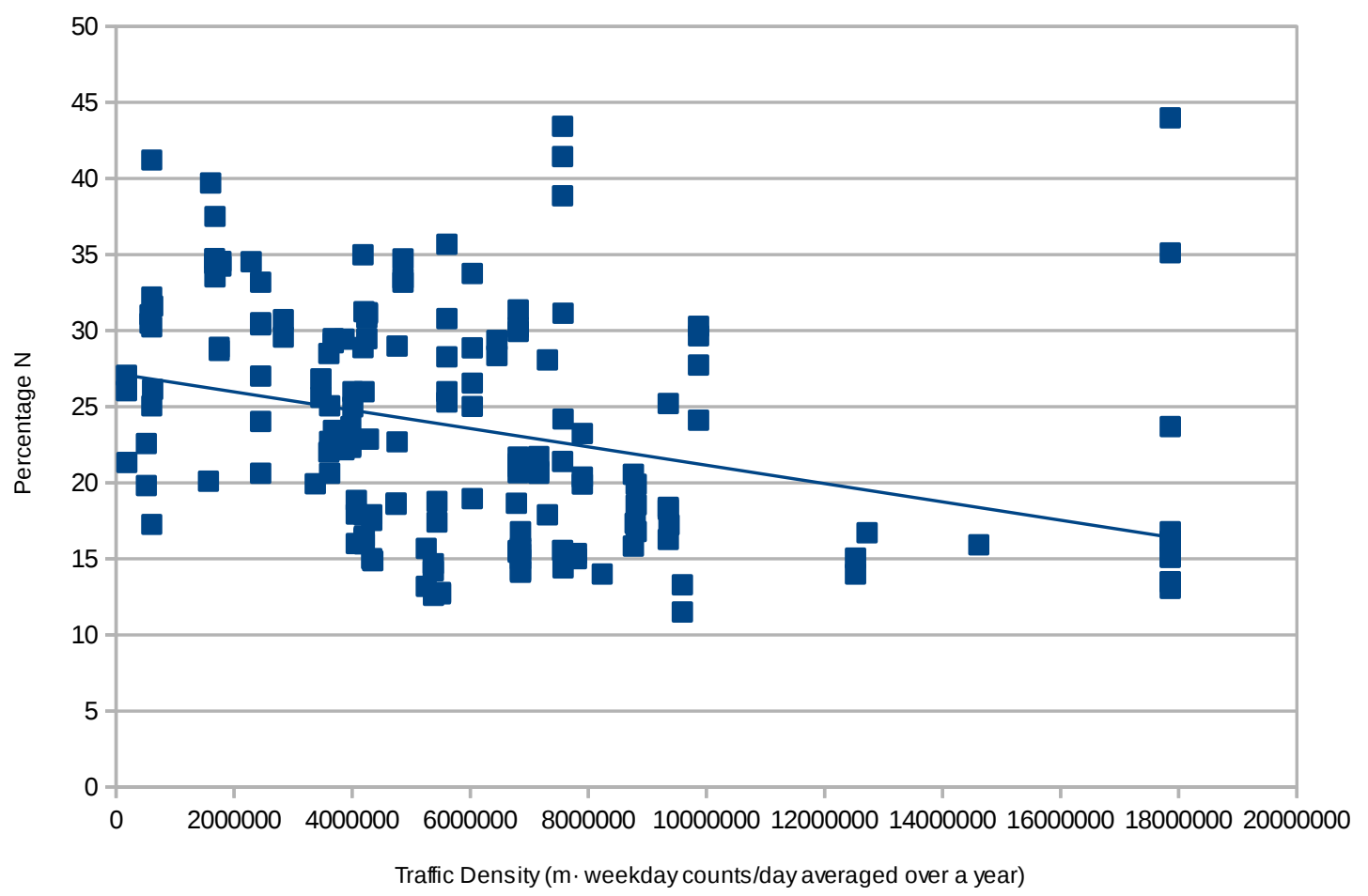

Figure 3.2: Plot of nitrogen content in moss vs. traffic counts. 
H04: Linear regression is appropriate for the traffic count $\S 3.0 .11$ data + our LUR statistics The ability to derive a meaningful linear regression from a data set depends on a number of rather restrictive characteristics of the data [43. These are neatly summarized in [46, pp. 412-413] as

1. Linearity.

2. Full rank: nonsingular predictor matrix.

3. Independent variables display exogenicity: $\epsilon$ 's mean is 0 for the full range of the independent variables.

4. Homoscedasticity (constant variance of $\epsilon$ ).

5. Nonautocorrelation between sample values.

6. Exogencity in the sampling: $\epsilon$ is not a product of any of the independent variables.

7. $\epsilon \sim N\left(0, \sigma^{2}\right)$

However, you can also produce useful linear models when there is some degree of violation of these requirements. We attempt to evaluate our data: does the data satisfy the seven requirements?

H05: Observing that the residuals of the linear regression

The QQ plots in the bottom panel of Figure 2.8 show a pretty linear feature over much of the data. We take this as good evidence of the homoscedasticity of the residuals after the log-transform. The graph of residuals in Figure 2.9 (b) lacks the definite linear funnel shapes of Figure 2.9 (a). We take this as further evidence that the residuals are homoscedastic after the log-transform. 
As discussed in section 2.8.3, we made a linear model using the well-understood least-squares regression method. Using the $\mathrm{lm}$ feature of $\mathrm{R}$, we fit the data to a model of $x$ formkount $r c+$ pop $100+$ pop $200+$ pop $300+$ pop $500+$ pop $600+$ pop $700+$ pop $900+$ or $100+$ or $200+$ or $300+$ or $400+$ or 500 , where xformkount is log-transformed traffic counts in units of natural log of the ADT.

The residuals mapped out fairly normal, slightly skewed to the negative, with $\min =-5.7738,1 \mathrm{Q}=-0.2286$, Median $-0.0117,3 \mathrm{Q}=0.2224$, and $\mathrm{Max}=3.6996$. The coefficients are given in Table 3.4 .

The regression produces an equation purporting to reproduce a linear trend in the raw data. A test exists against the null hypothesis that the slope of the regression line is actually zero. The significance factors in the coefficients Table 3.4 indicate that we have enough independent data to make a useful linear model involving fourteen road classes, seven population density radii, and five onramp mileage radii with $\alpha=0.05$.

H07: a linear regression can reproduce trends in the

The count data held back during the creation of the linear model were plotted against the prediction of the counts made by the model. This is considered to be the ultimate test of a linear regression. The result shows a good fit, with a slope of 1.008 and an $r^{2}$ of 0.90 . The Y-intercept is -0.09 , indicating a slightly skewed fit. The graph in Figure 3.3 gives an overview of the result.

The fit is perhaps more convincing when seen in a map. Figure 2.6 on page 25 presents the graphical view. 


\begin{tabular}{lrlrll} 
Parameter & Estimate & Std. Error & $\mathrm{t}$ value & Pr $(>a b s(t))$ & Significance Code \\
\hline (Intercept) & $1.108(+01)$ & $1.042(-02)$ & 1063.878 & $<2 \mathrm{e}-16$ & $* * *$ \\
rc1200 & $-3.602(-01)$ & $1.194(-02)$ & -30.172 & $<2 \mathrm{e}-16$ & $* * *$ \\
rc1221 & $-2.956(+00)$ & $9.070(-02)$ & -32.593 & $<2 \mathrm{e}-16$ & $* * *$ \\
rc1222 & $-2.572(+00)$ & $7.996(-02)$ & -32.172 & $<2 \mathrm{e}-16$ & $* * *$ \\
rc1300 & $-1.672(+00)$ & $1.882(-02)$ & -88.882 & $<2 \mathrm{e}-16$ & $* * *$ \\
rc1400 & $-2.106(+00)$ & $1.959(-02)$ & -107.472 & $<2 \mathrm{e}-16$ & $* * *$ \\
rc1450 & $-2.917(+00)$ & $1.722(-02)$ & -169.359 & $<2 \mathrm{e}-16$ & $* * *$ \\
rc1500 & $-4.746(+00)$ & $1.463(-02)$ & -324.383 & $<2 \mathrm{e}-16$ & $* * *$ \\
rc1521 & $-2.152(+00)$ & $3.573(-02)$ & -60.231 & $<2 \mathrm{e}-16$ & $* * *$ \\
rc1700 & $-3.340(+00)$ & $1.467(-01)$ & -22.768 & $<2 \mathrm{e}-16$ & $* * *$ \\
rc1800 & $-4.407(+00)$ & $2.144(-01)$ & -20.555 & $<2 \mathrm{e}-16$ & $* * *$ \\
rc2000 & $-7.318(+00)$ & $5.667(-01)$ & -12.912 & $<2 \mathrm{e}-16$ & $* * *$ \\
rc5301 & $-2.216(+00)$ & $4.027(-02)$ & -55.028 & $<2 \mathrm{e}-16$ & $* * *$ \\
rc5401 & $-3.004(+00)$ & $9.267(-02)$ & -32.420 & $<2 \mathrm{e}-16$ & $* * *$ \\
rc5501 & $-3.728(+00)$ & $5.544(-02)$ & -67.239 & $<2 \mathrm{e}-16$ & $* * *$ \\
pop100 & $-1.268(-02)$ & $1.404(-03)$ & -9.033 & $<2 \mathrm{e}-16$ & $* * *$ \\
pop200 & $4.125(-03)$ & $8.723(-04)$ & 4.729 & $2.27(-06)$ & $* * *$ \\
pop300 & $-1.308(-03)$ & $4.104(-04)$ & -3.188 & 0.001436 & $* *$ \\
pop500 & $1.862(-03)$ & $2.774(-04)$ & 6.711 & $1.98(-11)$ & $* * *$ \\
pop600 & $-1.008(-03)$ & $3.398(-04)$ & -2.966 & 0.003018 & $* *$ \\
pop700 & $-3.697(-04)$ & $1.923(-04)$ & -1.923 & 0.054517 & $*$ \\
pop900 & $2.519(-04)$ & $3.194(-05)$ & 7.888 & $3.20(-15)$ & $* * *$ \\
or100 & $-2.705(-04)$ & $6.146(-05)$ & -4.402 & $1.08(-05)$ & $* * *$ \\
or200 & $-1.834(-04)$ & $4.744(-05)$ & -3.867 & 0.000111 & $* * *$ \\
or300 & $6.071(-04)$ & $6.007(-05)$ & 10.107 & $<2 \mathrm{e}-16$ & $* * *$ \\
or400 & $-4.600(-04)$ & $5.344(-05)$ & -8.607 & $<2 \mathrm{e}-16$ & $* * *$ \\
or500 & $2.567(-04)$ & $2.349(-05)$ & 10.925 & $<2 \mathrm{e}-16$ & $* * *$
\end{tabular}

Table 3.4: Coefficients from the linear regression. The units are in the natural log of the ADT. The significance codes are $0^{(* * *}, 0.001^{(* *}, 0.01^{(*}, 0.05$ ' $\quad$ The multiple $\mathrm{R}^{2}$ is 0.8904 . 


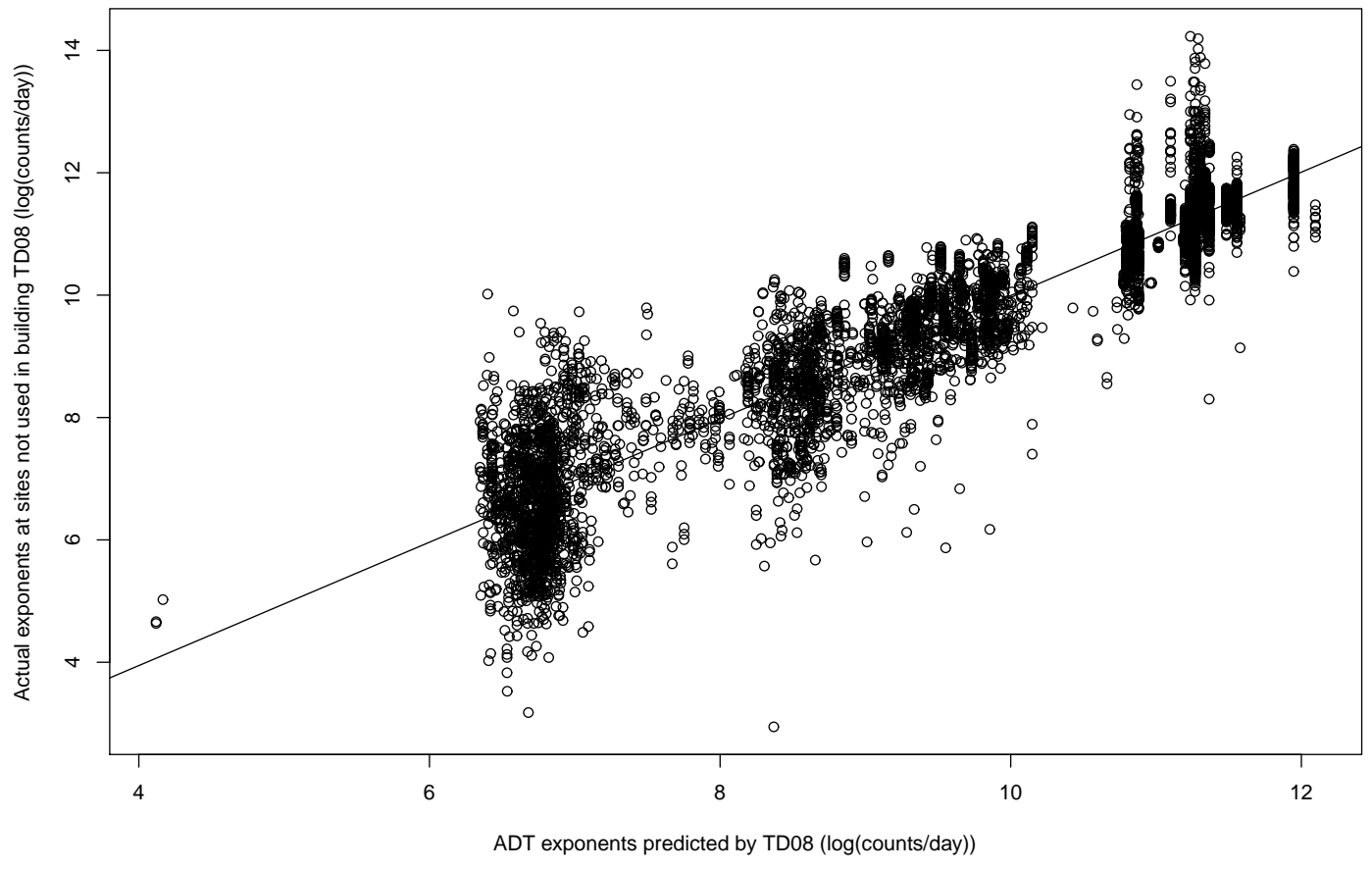

Figure 3.3: The actual count data in the set held back during model creation is plotted against the values for these counts predicted by the linear model. The equation of the line is $\mathrm{y}=-0.08613+1.00799 \mathrm{x}$. The $\mathrm{r}^{2}$ is 0.90 . 11,463 data points are plotted. 
Our goal was to accept only counting that was as clean as possible. Any aberration was taken as grounds to throw out the count. We believe that this succeeded and the product is quite clean. To test this hypothesis, we used a statistical test to search for outliers.

We used Cook's Distance as described in [55] to determine data that exert extreme leverage in the regression. The result is given in Figure 3.4. Metrics like Cook's Distance are best interpreted in the context of the distribution of the data, and best used in a graphical setting as we have done here instead of as a strict cutoff point. We are pleased to see in the figure that very few of our large number of records exert any particularly strong leverage on the regression coefficients.

H09: Rush hours will produce a bimodal trend in the diel, $\S 3.0 .17$ and weekends will be highly attenuated in counts.

We graphed the counts to search for this expected result. The results are shown in Figure 3.5. We note that the graph of Figure 3.5 would be misleading if the data included any days that did not include a full 24 hours of counting. In data cleaning we eliminated any counts that were not part of a full, clean 24 hours of counting so we can correctly interpret the graphical evidence of both the weekend effect and the bimodal diel cycle in Figure 3.5 without any misleading bias. The code for producing Figure 3.5 is in Appendix A.6.8.

We used a Welch Two Sample t-test to determine the probability that the weekend effect is present. We found a significant $(p<0.0001, \mathrm{t}=-12.4, \mathrm{df}=$ $1360)$ and large (23\%) drop off in traffic on the weekends for primary arterials. A significant $(p<0.001, \mathrm{t}=-27, \mathrm{df}=37428)$ and large $(22 \%)$ drop in traffic on the weekends is seen for a pooled mean of all of the road classes. The evidence 


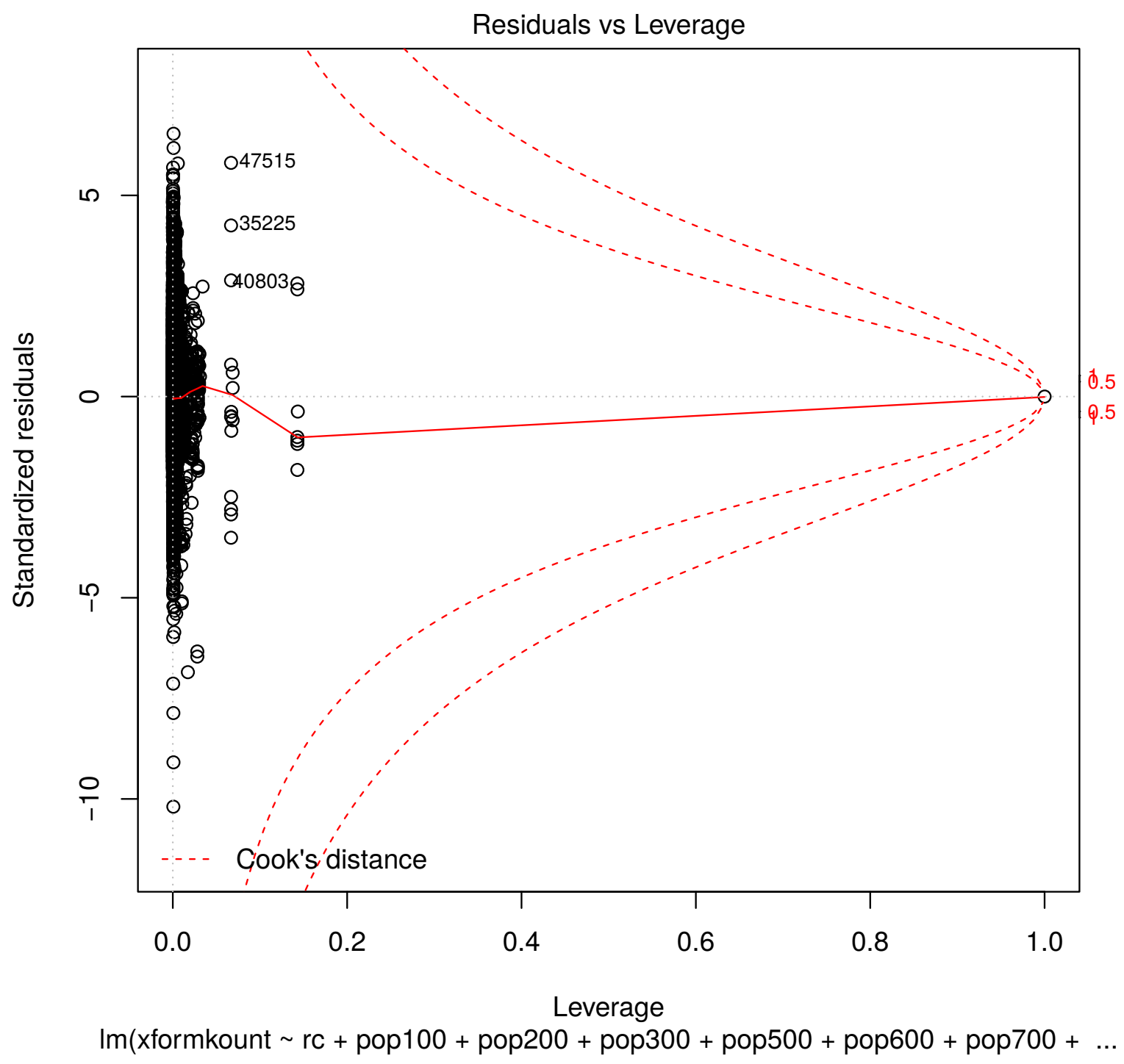

Figure 3.4: Leverage of the points used in the regression, with Cook's Distance marked. 


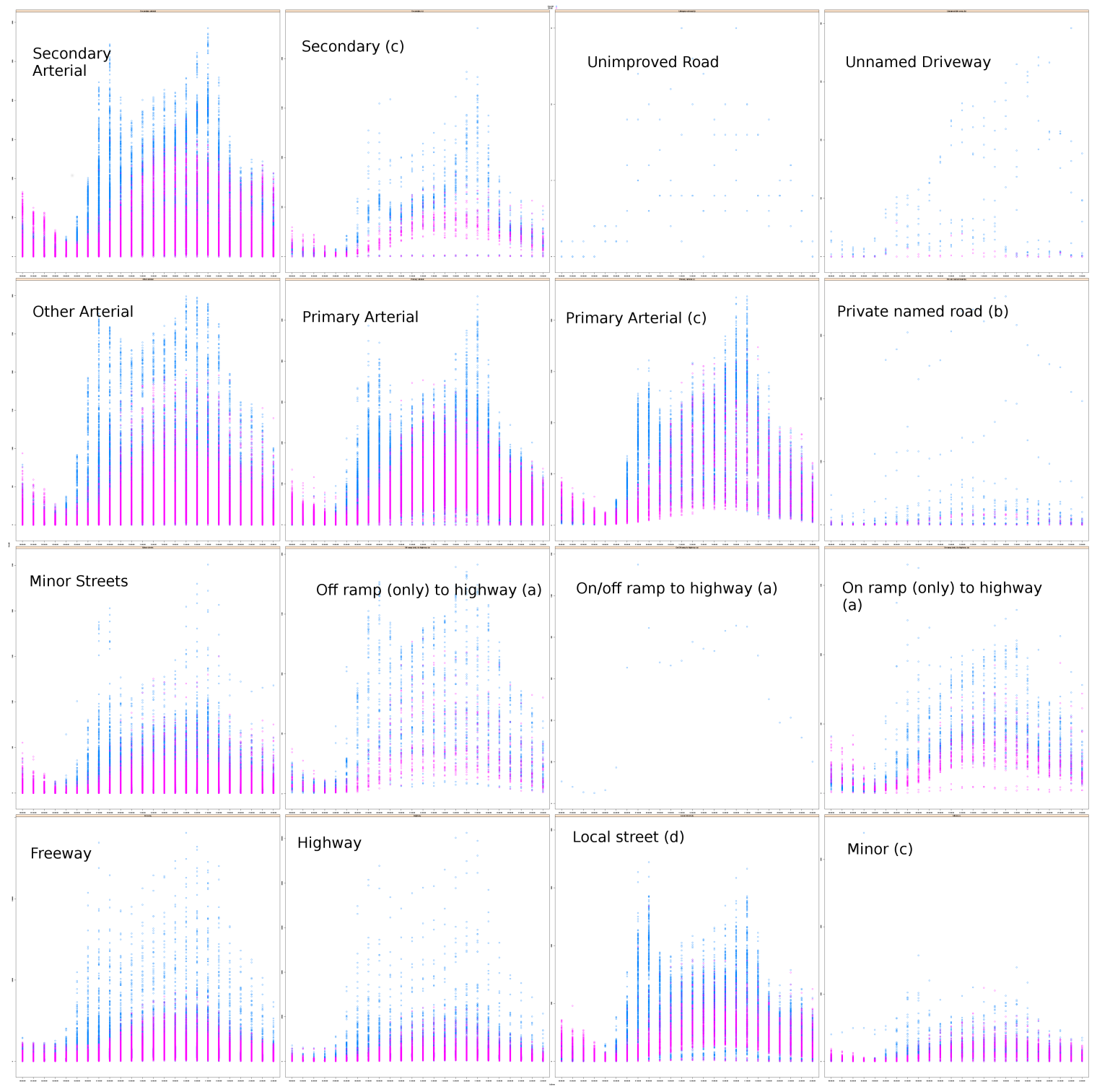

Figure 3.5: A plot showing the diurnal (diel) trends in the data. Each panel graphs all of the available data, as counts per day vs. the time of day (00:00-23:00). The blue colored dots are a single datum for each hourly binned count made on a week day, red are the same but for counts made on weekends only. (a) = "In the area maintained by the City of Portland only". (b) = "Private right-of-way exists". (c) = "With rapid transit". (d) = "To local street connector (Portland only, subarea = 'P')". $(\mathrm{e})=$ "Named, but without valid addressing (Clackamas County only, subarea = 'C')". (f) = "Unnamed and without valid addressing (Clackamas County only, subarea = 'C')". $(\mathrm{g})=$ "With valid address range and street name (in the area maintained by the City of Portland only)". (h) = "With NO Valid Address Range or Street Name (in the area maintained by the City of Portland only)". (i) = "No private rightof-way exists". (j) = "Passable by emergency vehicles (e-911) only (in the area maintained by the City of Portland only)". 
Results/Discussion

suggests that we can safely reject the null hypothesis that there is no difference in ADT mean between weekends and weekdays. The code for the weekend test is in Appendix A.6.3.

H10: Small seasonal differences are expected.

To test for seasonal differences, we partitioned the data by season, defining winter as December, January, and February. Spring is March, April, May. Summer is June, July, and August. Fall is September, October, and November.

We tested the mean of the average of all of the data for primary arterials against the mean of the subset of the data in each season using the Welch Two Sample t-test. We found a small $(7.8 \%)$ and significant $(p<0.001, t=-3.64, d f=776)$ enhancement in traffic volume during the winter. We found a small (5.9\%) and significant $(p<0.01, t=2.69, d f=877)$ decrease in traffic volume during the spring. We found a small $(7.2 \%)$ and significant $(p<0.001, t=3.34, d f=7944)$ decrease in traffic volume during the summer. We found no significant difference in the mean fall traffic $(p=0.064, t=-1.85, d f=1057)$ vs. the overall mean.

H11: Our EI compares well with other, independent

estimates

Our estimate was compared to other published estimates. The number we used for comparison are:

- 9.1E7 metric ton (MT) $\mathrm{CO}_{2}$ emitted (average quantity for 1986-2006 (unrestricted) and 2005-2014 (restricted) from our own Circoscope inventory calculated using local VMT and a national average EF.

- 9.8E7 $\mathrm{MT} \mathrm{CO}_{2}$ e of GHGs emitted in 2001 (our own work extracting the Portland region's pixels from Vulcan [5] ) 


\subsection{LIMITATIONS}

- 2.5E7 MT $\mathrm{CO}_{2}$ e of GHGs emitted in 2010 [56].

- 6.1E6 MT of GHGs emitted in 2005 - Metro (Portland's regional authority) estimate. 1

Our estimate agrees closely with Vulcan's, and remains about ten times higher than the City of Portland and Metro's estimates, even though the latter included non- $\mathrm{CO}_{2}$ gasses. This is typical of EI work: finding the best number is difficult and this motivates the WRF ground truthing of our Future Work (Section 5).

Our work is limited to just one mode (on-road transport), as is seen in other works of the genre [57] [18] [54] [58. We cannot complete an emissions inventory for $\mathrm{CO}_{2}$ without including other important sources such as off-road transport and residential and commercial fossil fuel use.

We are limited also to tank-to-wheel emissions, so our inventory does not include well-to-tank emissions, defined as "emissions from the extraction of primary energy carriers, and their conversion, refinement and delivery" [57, p. 7609] which are important for the purposes of mitigation.

We present results related to $\mathrm{CO}_{2}$ only, but as Nickless et. al. write [57, other species with shorter lifetimes cause global warming (NOx, ozone, contrails and cirrus clouds) and are relevant to the problem of climate change which motivates this study and must be included in the comprehensive picture to enable the creation of useful short-term plans which are themselves a necessary complement to longterm plans.

\footnotetext{
${ }^{1}$ We multiplied "The average Portland-area resident emitted 4.05 tons of carbon dioxide and other greenhouse gases in 2005" from (http://www.oregonmetro.gov/news/ tailpipe-emissions-portland-region-climate-smart-communities-qa accessed 2016-10-06) by the quantity of "1,500,000 people Metro serves" from (http://www.oregonmetro.gov/regional-leadership accessed 2016-10-16).
} 
We calculate AADT for comparison with [51] simply by taking the arithmetic mean of the year's traffic. This is potentially biased because the counters are preferentially taken down for maintenance on certain days of the week. A better approach is described in [59, p. 1-5], creating an average of averages.

Uncertainties

Here we identify several parts of the research that introduce uncertainty.

\section{TDAT - conditions}

TDAT identifies a handful of conditions marking a count as unusual. These are recorded as "conditions" in a table called public.volcount, and include values such as "Bike Path Only" and "Tax Day at the Post Office."

These condition-marked records are not presently excluded as outliers in the analysis. These condition-marked records appear to be outliers by the descriptive text just above, and the associated count records should be excluded as outliers, except possibly the synaptic ones.

\section{Bikes being Counted}

In Portland, there is a significant biking population. It is not known at this time whether bikes are counted along with cars by the automated traffic recorder (ATR)s that the city uses and thus are included in our count.

\section{One Way Streets}

We do not notice a street that is one way. As a result our ability to predict ADT is diminished; we underpredict two-way streets and overpredict one-way. 


\subsection{LIMITATIONS}

\section{Miscoding of Weekends}

If TDAT classifies a weekend as a weekday (or vice-versa), this would indicate some confusion in the record and we could detect that and use it as a reason to treat the day's count as an outlier. This would be done in Pass 1, where other exceptType (excepttype $\neq$ "normal weekday" and excepttype $\neq$ "normal weekend") are already marked as outliers.

\section{Multiple, Independent Counts on one Site/Day}

If there are instances of multiple, independent counts of the same place on the same day, we do not catch those currently and so over count.

\section{Autocorrelation}

To avoid the problem of autocorrelation, which we discussed in Section 3.0.11, in light of the results of Sections 3.0.17 and 3.0.18 it would be more accurate to use eight different equations of the form of Equation (1.1), one for each possible combination of weekend/weekday and season. 
Conclusion

Chapter 4

Conclusion

A new model of ADT for the North American city of Portland, OR, USA has been created using multiple linear regression against a collection of 1.1 million full days of counting at a diverse array of 7,000 regional sites over the period spanning the years 1986-2006 (unrestricted roads) and 2005-2014 (restricted roads), supplemented by "land use" statistics for population density and extent of restricted road on-ramp in the area around each count site, and by identification of the functional road class (arterial, local, etc.) of the count sites.

The model was tested by holding back one-third of the data and using the model results to estimate ADT at each of the held-back count sites. The result appears to validate the model, producing a fit with a slope of 1.008 and an $r^{2}$ of 0.90 .

We analyzed each road segment in a detailed map of the city by finding a centroid for each uninterrupted stretch of road (105,178 road segments). At each centroid, our "land use" variables (population density and on-ramp mileage) were evaluated using 250 CPUs in parallel on the PSU Gaia computing cluster.

These data were used with the model to estimate ADT for each road segment. The model ADT was multiplied by the road segment length to obtain VMT. This result is expected to be generally useful in building emissions inventories related to transport, meeting a current research need for high resolution emissions inventories to complement high resolution atmospheric models and neighborhood- or street segment- level policy-making decisions.

As a preliminary foray into the use of the data, the VMT values were mul- 
tiplied by a nationwide-average $\mathrm{CO}_{2} \mathrm{EF}$ to estimate daily $\mathrm{CO}_{2}$ emissions from on-road traffic. A city-wide EI for $\mathrm{CO}_{2}$ was made by summing and scaling the road segment emission estimates from the model, and the resulting sum compared to other estimates. Our result closely agrees with another, the Vulcan inventory, which is a factor of ten greater than estimates made by the City of Portland and by Metro, the regional authority.

We demonstrated the construction of a $1 \mathrm{~km} \mathrm{x} 1 \mathrm{~km}$ gridded inventory of $\mathrm{CO}_{2}$ emissions using the nationwide-average EF and the model. The result appear to be useful, correctly highlighting freeways as $\mathrm{CO}_{2}$ hotspots.

We used our model to search for a correlation between high ADT and high concentrations of nitrogen sampled in moss plants, but we have not been unable to establish the correlation. 
Future Work

Chapter 5

Future Work

Cities have a pressing need for aid in mitigating $\mathrm{CO}_{2}$ emissions, and the TD0N model offers a window onto the city's emissions landscape at a high spatial and temporal resolution.

In his talk at AGU in 2014, K. Gurney reported a discovery of a small area of Salt Lake City which is responsible for a large portion of the city's $\mathrm{CO}_{2}$ emissions. We plan to search for this effect in Portland.

The high resolution of our model enables our inventory to be used with WRF, a research-grade meteorological model, to predict $\mathrm{CO}_{2}$ concentrations anywhere in the region. We will use WRF-GHG to generate model time series at high temporal resolution and compare these to tower measurements, ground truthing TD0N. We will acquire a map of biogenic emissions (perhaps Model of Emissions of Gases and Aerosols from Nature (MEGAN)) to supply this component of the carbon flux.

Top-down methods validate bottom-up methods [60] and our work will carry out this validation step in the future, using an existing data set of $\mathrm{CO}_{2}$ concentration recordings made by Dr. Andrew Rice's group at three locations around town, locations chosen to sample a wide range of population densities.

We will extend our comparison to include the Emission Database for Global Atmospheric Research (EDGAR) 61] EI, the Highway Performance Monitoring System (HPMS) [11, Supp. p. 16] EI, the Vulcan [5] EI, and the National Emissions Inventory (NEI) 18. We have developed a method to create gridded EI at various resolutions, for example the $1 \mathrm{~km}$ EI of Figure 5.2. This type of summary result 


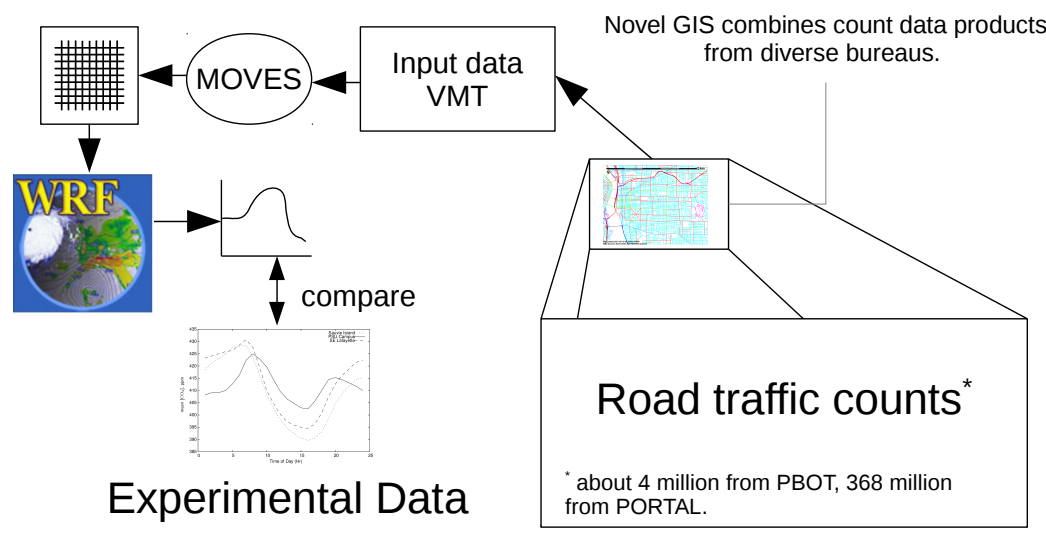

Figure 5.1: Conceptual model of the WRF components to the research. 


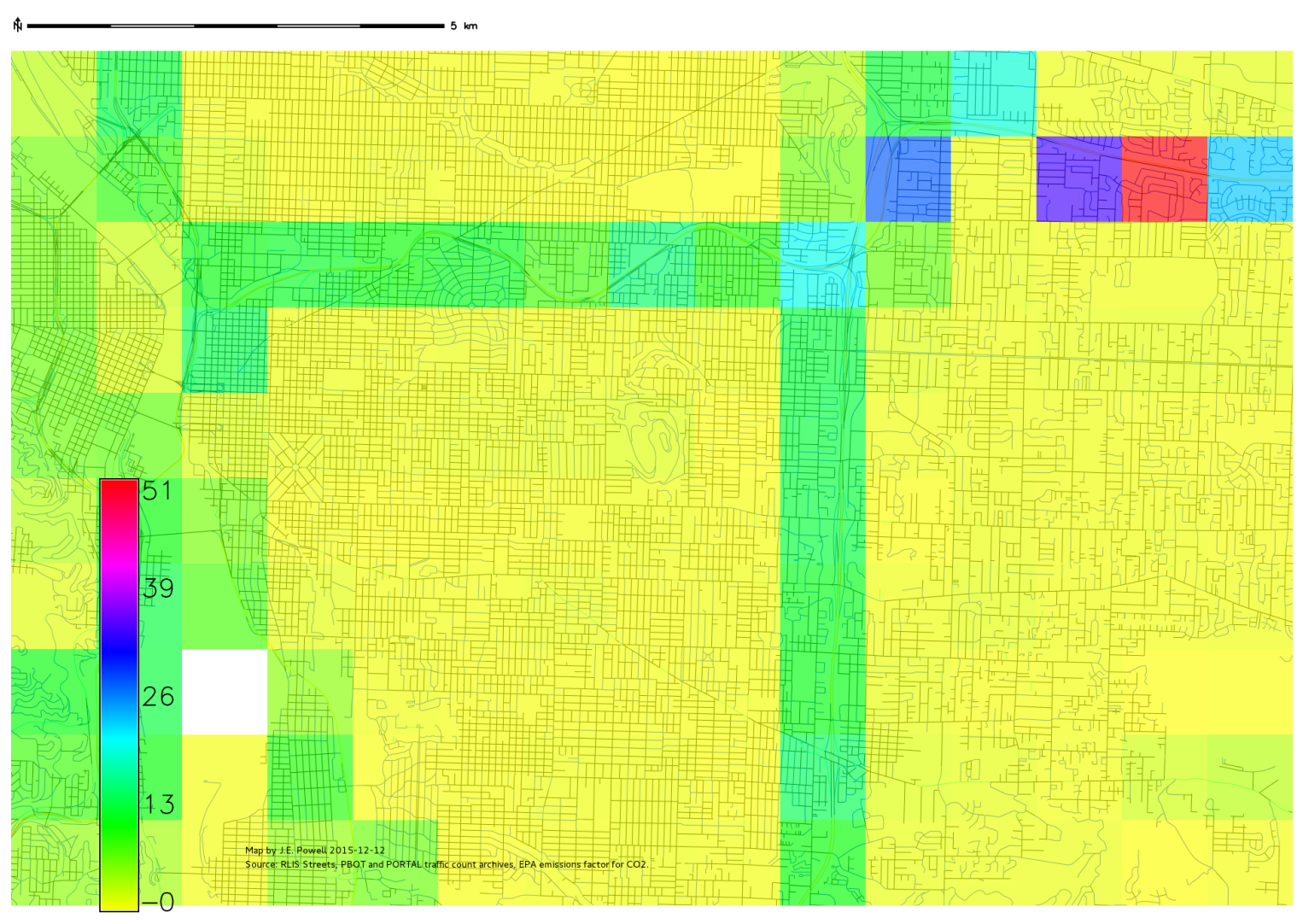

Figure 5.2: A $1 \mathrm{~km}$ resolution gridded $\mathrm{CO}_{2}$ emissions inventory for Portland, OR developed using our model. We presented this result at the GEIA conference in Beijing, December 2015. The EI shows the expected high intensity of emissions along the eastwest I-84 corridor (straight line, top center) and the north-south I-205 corridor (straight line, center right), zero emissions on an all-river pixel (the white pixel at bottom left), and fairly high emissions over downtown (top-left). The units are $\mathrm{MT}$ of $\mathrm{CO}_{2} /$ year.

makes for easy comparison with other gridded EIs and is typical of this type of research; for example [27] compare their result with EDGAR and Vulcan, and 18] compare their result with EDGAR, Vulcan, and HPMS.

We will repeat the moss $\mathrm{N}$ experiment, with more information than before, to find a signal.

We will continue to receive updates from PBOT and PORTAL about new traffic counts. We have completed one cycle of updates from PBOT already with no 
problems, and we anticipate given our flexible data structures (CSV or SQL into an RDBMS, and then materialized views to add structure to flat data) that we can accommodate even alternative forms of the data as the archives change over time.

A novel aspect of our work is its exploration of the Portland, OR metropolitan region, which has been ranked as the "most compact" city in the USA [62]. More realistically, Portland is found to rank 80th out of 221 US cities in the "Measuring Sprawl 2014" index 63. Portland ranks fifth the nation for light rail ridership. From 1998-2008, the average miles driven by a citizen of Portland remained flat at 20 miles a day, even while national average per-capita miles driven daily climbed steeply ${ }^{1}$. We expect to explore some hypotheses relating the city's lack of sprawl to the $\mathrm{CO}_{2}$ emissions inventory, such as the possibility that $\mathrm{CO}_{2}$ from on-road traffic should not be seen to grow linearly with the growth of the population but rather should lag somewhat behind it.

We will explore a hypothesis posed by Daniel Mendoza, who reviewed our work at AGU 2014, that Portland's Urban Growth Boundary should cause a visible effect by which population grows over time while freeway traffic holds constant. We believe other theories relating to Portland's progressive urban planning can be fruitfully explored through the high resolution TD0N model. We have concerns that super-emitters, real outliers such as diesel construction vehicles (bulldozers, cranes, dump trucks) or supercharged classic cars 2? will simultaneously not be captured by our model and yet represent a majority of emissions, a known problem for some pollutants today. We will learn whether this is true, because it will make calibration of the model with tower measurements difficult.

\footnotetext{
1 http://www.oregonlive.com/environment/index.ssf/2008/05/portland_reduces_its_carbon_ fo.html accessed 2016-10-06

${ }^{2}$ This is a hobby which is gaining in popularity in Portland. Walking home one Sunday evening in October 2016, I encountered a train of some thirty vehicles, all extremely loud, emerging from a weekly gathering. A passerby told me about it, that it's a growing movement coming up to Portland from areas such as Auburn in northern California.
} 
Another concern is that the roughness of the urban terrain defeats the ability of WRF to model air flow over the city. This defect is being addressed, with some success, by a group at Berkeley, CA who have modified WRF to include a new module feeding the boundary outputs of very turbulent low-altitude activity into the higher (but still low-altitude) boxes of the WRF model.

Our group has already encountered a problem of this sort in doing basic WRF validation against recorded air-speed measurements: WRF simply would not run faced with the sheer altitude gains of the nearby Coast and Cascade mountain ranges.

Another concern is the very different diel patterns expected of light duty (LD) vs. heavy duty (HD) traffic, and rural vs. urban traffic as found in [27.

Our approach has also been accurately criticized as being "traditional" 3 . In an age when sensors are pervasive and capable, using the ATR devices is limiting. It is possible, for example, for a camera to film license plates, and for those plates to be looked up in the registration records to find the year, model, and fuel type of the vehicle. Additionally, it is possible today to mine cell phone location data for velocity and traffic density. More work needs to be done in this area.

$\mathrm{C}$

\footnotetext{
${ }^{3}$ Leonor Tarrason speaking at GEIA 2015 in Beijing, China. Our EI of Figure 5.2 was projected on the big screen as an example of current work.
} 
Bibliography

[1] R Core Team. R: A Language and Environment for Statistical Computing. R Foundation for Statistical Computing. Vienna, Austria, 2015. URL: https ://www.Rproject.org.

[2] Deepayan Sarkar. Lattice : multivariate data visualization with $R$. [London]: London : Springer, 2008,

[3] TF Stocker et al. "IPCC, 2013: climate change 2013: the physical science basis. Contribution of working group I to the fifth assessment report of the intergovernmental panel on climate change". In: (2013). URL: http://www . ipcc.ch/pdf/ assessment-report/ar5/wg1/WG1AR5_ALL_FINAL.pdf.

[4] Juerg M Matter et al. "Rapid carbon mineralization for permanent disposal of anthropogenic carbon dioxide emissions". In: Science (New York, N.Y.) 352.6291 (2016), pp. 1312-.

[5] Kevin R. Gurney et al. "High Resolution Fossil Fuel Combustion $\mathrm{CO}_{2}$ Emission Fluxes for the United States". In: Environmental Science 85 Technology 43.14 (2009), pp. 5535-5541. DOI: 10 . 1021/es900806c eprint: http : //pubs . acs . org/doi/pdf/10.1021/es900806c.

[6] Patricia Romero-Lankao et al. "A critical knowledge pathway to low-carbon, sustainable futures: Integrated understanding of urbanization, urban areas, and carbon". In: Earth's Future 2.10 (2014), pp. 515-532. ISSN: 2328-4277. 
[7] D. Mendoza et al. "Implications of uncertainty on regional $\mathrm{CO}_{2}$ mitigation policies for the U.S. onroad sector based on a high-resolution emissions estimate". In: Energy Policy, 55:386-395 (2013),

[8] James Powell, Chris L. Butenhoff, and Andrew L. Rice. Network Level Carbon Dioxide Emissions From On-road Sources in the Portland OR, (USA) Metropolitan Area. Poster at AGU, A53L-3370. 2014.

[9] Ian Stewart, Jack S. (Jack Sidney) Cohen, and Terry Pratchett. The science of Discworld. 2, The globe. Ed. by Ian Stewart and Jack S. (Jack Sidney) Cohen. London: London : Ebury, 2003.

[10] Sam Adams and Jeff Cogen, eds. Climate Action Plan 2009. City of Portland, 2009.

[11] Conor K Gately, Lucy R Hutyra, and Ian Sue Wing. "Cities, traffic, and $\mathrm{CO}_{2}$ : A multidecadal assessment of trends, drivers, and scaling relationships". In: Proceedings of the National Academy of Sciences of the United States of America 112.16 (2015), pp. 4999-. URL: http://www.pnas.org/content/112/16/4999.

[12] California Legislature. "California Global Warming Solutions Act (AB 32), Health \& SC $\S 38500-38598 " .2006$. URL: http://www.arb.ca.gov/cc/docs/ab32text. pdf.

[13] Barack Obama. White House press release. Accessed 2016-10-08. Tech. rep. 2014. URL: https : / / wwW . whitehouse .gov/the-press-office/2014/11/11/factsheet-us-china-joint-announcement-climate-change-and-clean-energyC.

[14] Kathryn McKain et al. "Assessment of ground-based atmospheric observations for verification of greenhouse gas emissions from an urban region". In: Proceedings of the National Academy of Sciences of the United States of America 109.22 (2012), pp. $8423-$. 
[15] Stephen M Wheeler. "State and municipal climate change plans: the first generation". In: Journal of the American Planning Association 74.4 (2008), pp. 481496.

[16] Elizabeth C. Brodeen. "Sequestration, science, and the law: an analysis of the sequestration component of the California and northeastern states' plans to curb global warming". In: Environmental Law 37.4 (2007), p. 1217. ISSN: 0046-2276.

[17] Philippe Ciais et al. "The European carbon balance. Part 1: fossil fuel emissions". In: Global Change Biology 16.5 (2009). ISSN: 1354-1013.

[18] Conor K Gately et al. "A Bottom up Approach to On-Road $\mathrm{CO}_{2}$ Emissions Estimates: Improved Spatial Accuracy and Applications for Regional Planning". In: Environmental Science \&6 Technology 47.5 (2013), pp. 2423-2430.

[19] EPA. U.S. Greenhouse Gas Inventory Report: 1990-2013. Tech. rep. U.S. Government, 2015. URL: http://www3 . epa . gov/climatechange/ghgemissions / usinventoryreport.html.

[20] Carolyn Kousky and Stephen H Schneider. "Global climate policy: will cities lead the way?" In: Climate Policy 3.4 (2003), pp. 359-372.

[21] Felix Creutzig et al. "Energy and environment. Transport: A roadblock to climate change mitigation?" In: Science (New York, N.Y.) 350.6263 (2015), pp. 911-.

[22] Ralph Sims et. al. Intergovernmental Panel on Climate Change. Working Group III- Mitigation of Climate Change. Chapter 8 Transport. 2014. DOI: 10.13140/2. 1.4480 .3521 .

[23] Lee Chapman. "Transport and climate change: a review". In: Journal of transport geography 15.5 (2007), pp. 354-367.

[24] Battelle. Traffic Data Quality Measurement - Final Report. Tech. rep. p. A-8, Table A.1. Completeness Statistics for Original Source Data. Office of Highway Policy 
Information Federal Highway Administration U.S. Department of Transportation Washington, D.C., 2004.

[25] Kristin Tufte and et. al. PORTAL. Tech. rep. PSU, 2013.

[26] Kevin R Gurney et al. "Quantification of fossil fuel $\mathrm{CO}_{2}$ emissions on the building/street scale for a large US City". In: Environmental Science \& Technology 46.21 (2012), pp. 12194-12202.

[27] Brian C. Mcdonald et al. "High?resolution mapping of motor vehicle carbon dioxide emissions". In: Journal of Geophysical Research: Atmospheres 119.9 (2014), pp. 5283-5298. ISSN: 2169-897X.

[28] Harry Smith and Norman Richard Draper. Applied regression analysis. New York: New York : Wiley, 1981.

[29] Patrick H. Ryan and Grace K. LeMasters. "A Review of Land-use Regression Models for Characterizing Intraurban Air Pollution Exposure.” In: Inhalation Toxicology 19 (2007), pp. 127-133. ISSN: 08958378. URL: http://stats.lib.pdx.edu/proxy. php?url=http : // search . ebscohost . com/login . aspx? direct =true\&db=hch\& $\mathrm{AN}=26641305 \&$ site=ehost - live.

[30] Manju Mohan, Lalit Dagar, and BR Gurjar. "Preparation and validation of gridded emission inventory of criteria air pollutants and identification of emission hotspots for megacity Delhi". In: Environmental monitoring and assessment 130.1-3 (2007), pp. $323-339$.

[31] Pathmathevan Mahadevan et al. "A satellite-based biosphere parameterization for net ecosystem $\mathrm{CO}_{2}$ exchange: Vegetation Photosynthesis and Respiration Model (VPRM)". In: Global Biogeochemical Cycles 22.2 (2008). GB2005, n/a-n/a. ISSN: 1944-9224. DOI: 10.1029/2006GB002735

[32] Thomas Lauvaux et al. "Urban emissions of $\mathrm{CO}_{2}$ from Davos, Switzerland: the first real-time monitoring system using an atmospheric inversion technique". In: 
Journal of Applied Meteorology and Climatology (Aug. 21, 2013). ISSN: 1558-8424. DOI: $10.1175 /$ JAMC-D-13-038.1.

[33] Society of American Archivists. Describing archives : a content standard. Chicago : Society of American Archivists, 2013.

[34] Kathleen D. Roe. Arranging 86 Describing Archives 85 Manuscripts. The Society of American Archivists, Chicago IL, 2005.

[35] Jerome S. Bruner. "The act of discovery". In: Harvard Educational Review 31 (1961), pp. 21-32. URL: http://hal .archives-ouvertes.fr/hal-00692072.

[36] Grady Booch, James Rumbaugh, and Ivar Jacobson. The Unified Modeling Language User Guide. Addison Wesley, 1998.

[37] F. Codd E. "A relational model of data for large shared data banks". In: Communications of the ACM 13.6 (1970). Ed. by M. Lynn, pp. 377-387. ISSN: 0001-0782.

[38] Annette J. Dobson. An introduction to generalized linear models. London; New York: Chapman and Hall, 1990.

[39] Herbert Friedman. Introduction to Statistics. Random House, Inc., New York, 1972.

[40] Robert L. Bertini Kristin A. Tufte. PORTAL Data Quality Analysis. Tech. rep. PSU, 2008. URL: http://www .its.pdx.edu/upload_docs/1248894239sryN2wqBix. pdf.

[41] John R. (John Robert) Taylor. An introduction to error analysis : the study of uncertainties in physical measurements. Mill Valley, Calif.: Mill Valley, Calif. : University Science Books, 1982,

[42] Martin B Wilk and Ram Gnanadesikan. "Probability plotting methods for the analysis for the analysis of data". In: Biometrika 55.1 (1968), pp. 1-17.

[43] R. Dennis Cook and Sanford Weisberg. Residuals and influence in regression. Ed. by Sanford Weisberg. New York: New York : Chapman and Hall, 1982. 
[44] Md Shohel Reza Amin. "Application of Spatial Auto-Regressive Model for Determining Urban Land Market". In: Journal of Bangladesh Institute of Planners 2 (2009), pp. 107-115.

[45] William G. (William Gemmell) Cochran and George W. (George Waddel) Snedecor. Statistical methods. Ed. by William G. (William Gemmell) Cochran. Ames, Iowa: Ames, Iowa : Iowa State University Press, 1967.

[46] Joseph Adler. $R$ in a Nutshell. O'Reilly Media, 2009.

[47] John M. Chambers and Trevor Hastie. Statistical models in S. Ed. by John M. Chambers and Trevor Hastie. Pacific Grove, Calif.: Pacific Grove, Calif. : Wadsworth \& Brooks/Cole Advanced Books \& Software, 1992.

[48] Dallas E. Johnson and George A. Milliken. Analysis of messy data. Ed. by Dallas E. Johnson. Belmont, Calif.: Belmont, Calif. : Lifetime Learning Publications, 1984.

[49] John I. (John Ignatius) Griffin. Statistics : methods and applications. New York: New York : Holt, Rinehart and Winston, 1962.

[50] Antoine Waked, Charbel Afif, and Christian Seigneur. "An atmospheric emission inventory of anthropogenic and biogenic sources for Lebanon". In: Atmospheric Environment 50 (2012), pp. 88-96. ISSN: 1352-2310.

[51] Christine M. Kendrick, Peter Koonce, and Linda A. George. "Diurnal and seasonal variations of $\mathrm{NO}, \mathrm{NO}_{2}$ and $\mathrm{PM}_{2.5}$ mass as a function of traffic volumes alongside an urban arterial". In: Atmospheric Environment 122 (2015), pp. 133-141. ISSN: $1352-2310$.

[52] M. B. (Maurice Bertram) Priestley. Non-linear and non-stationary time series analysis. San Diego: San Diego : Academic Press, 1988.

[53] Stuart Allan et al. Atlas of Oregon. Ed. by Stuart Allan, Aileen R Buckley, and James E Meacham. Eugene, Or., 2001. 


\section{BIBLIOGRAPHY}

[54] Max N. Brondfield et al. "Modeling and validation of on-road $\mathrm{CO}_{2}$ emissions inventories at the urban regional scale". In: Environmental Pollution 170 (2012), pp. 113-123. ISSN: 0269-7491. DOI: 10.1016/j.envpol.2012.06.003. URL: http: //www.sciencedirect.com/science/article/pii/S0269749112002783

[55] John Fox. Regression Diagnostics. Newbury Park, Calif.: Newbury Park, Calif. : Sage Publications, 1991.

[56] C.O.P. City of Portland Carbon Dioxide Reduction Strategy Update. Tech. rep. City of Portland, Nov. 1997.

[57] Alecia Nickless, Robert J. Scholes, and Ed Filby. "Spatial and temporal disaggregation of anthropogenic $\mathrm{CO}_{2}$ emissions from the City of Cape Town". In: (Nov. 2015). DOI: 10.17159/sajs.2015/20140387. URL: http://wwW.sajs.co.za/spatialand-temporal-disaggregation-anthropogenic-co2-emissions-city-capetown/alecia-nickless-robert-j-scholes-ed-filby

[58] Yuqin Shu and Nina S.N. Lam. "Spatial disaggregation of carbon dioxide emissions from road traffic based on multiple linear regression model". In: Atmospheric Environment 45.3 (Jan. 2011), pp. 634-640. ISSN: 1352-2310. URL: http://www. sciencedirect.com/science/article/pii/S1352231010009234.

[59] Highway Performance Monitoring System Field Manual. Federal Highway Administration. Mar. 2014. URL: http: / / www . fhwa . dot . gov/policyinformation / hpms/fieldmanual/

[60] Gregory J. Frost et al. "New Directions: GEIA's 2020 vision for better air emissions information". In: Atmospheric Environment 81 (Dec. 2013), pp. 710-712. ISSN: 1352-2310. URL: http://wWw.sciencedirect.com/science/article/pii/ S1352231013006833

[61] Johannes Gerardus Jozef Olivier et al. Description of EDGAR Version 2.0: A set of global emission inventories of greenhouse gases and ozone-depleting substances 
for all anthropogenic and most natural sources on a per country basis and on 1 degree $x 1$ degree grid. 1996.

[62] R Ewing, R Pendall, and D Chen. "Measuring sprawl and its transportation impacts". In: Travel Demand And Land Use 20031831 (2003), pp. 175-183. ISSN: 0361-1981.

[63] Reid Ewing and Shima Hamidi. Measuring Sprawl 2014. Tech. rep. Accessed 201511-25. Smart Growth America, 2014. URL: http://www. smartgrowthamerica. org/measuring-sprawl.

[64] J. Frank Cook, Society of American Archivists. College, and University Archives Committee. Forms manual. Ed. by J. Frank Cook. Madison, Wisc.: Madison, Wisc., 1973.

[65] Michel Duchein. "Theoretical Principles and Practical Problems of Respect des fonds in Archival Science". In: (1983).

[66] Erich Gamma et al. Design Patterns. Addison-Wesley, Reading MA, 1995.

[67] EPA. Using MOVES for Estimating State and Local Inventories of On-Road Greenhouse Gas Emissions and Energy Consumption. E, 2012. 
Cleaning these two separate, different, and massive traffic count databases has been a great challenge. We reduced $14 \mathrm{~GB}$ of data - collected over many years with errors, missing data, redundant counts, and every creative possible misuse of the model system present. This has stressed the very limits of our data preparation abilities. It required years of dedicated labor. The end result is a clean, small, logical table of counts and the ability to aggregate reliable counts at the hourly or daily level, and a table of outliers (Tables 3.2 and 3.3 with reasons given for each of the problem cases.

A pipeline was conceived and written out in ANSI standard flowchart code, reproduced here in Figures A.1, A.2, A.3, and A.4.

We learned through experiment that this type of engineering, taking the problem down to small easily digestible and explained steps is far superior to any attempt to combine the steps into one monolithic pass across the data. 
FILE:

tdon-master.dia

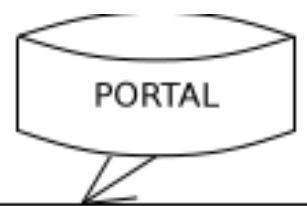

TACHE

Pipeline

J. Powell

2016-2-22

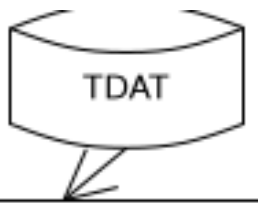

Pass 1

Throw out some outliers: locations that were blacklisted during Pass2 in an earlier run, and manually-entered locations (.is_locked is true in the outlier table)

- Find locations not blacklisted

\section{Pass 2}

Find Location/Counts not previously blacklisted

- Find locations with 0 counts

- Find PORTAL locations (aka stations)

with no "other" - these sit on one side of the

fwy with no companion monitoring flow in the other direction.

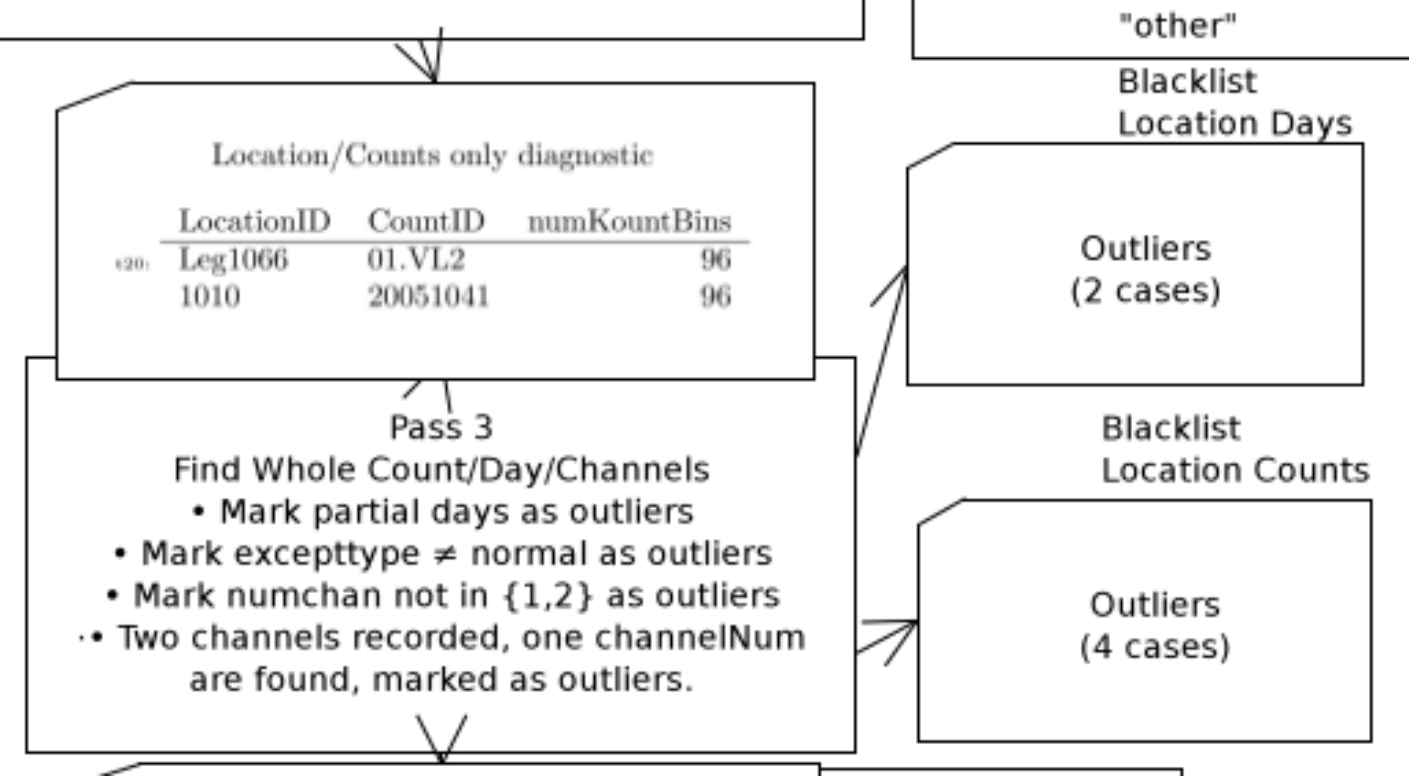

Figure A.1: TD0N pipeline, 1/4.
Blacklist

Locations

Outliers

- Locations with zero counts or with counts but no kounts

- PORTAL stations with no "other"

Blacklist Location Days

Outliers

Blacklist

Location Counts

4 cases)
( 2 cases) are found, marked as outliers. 


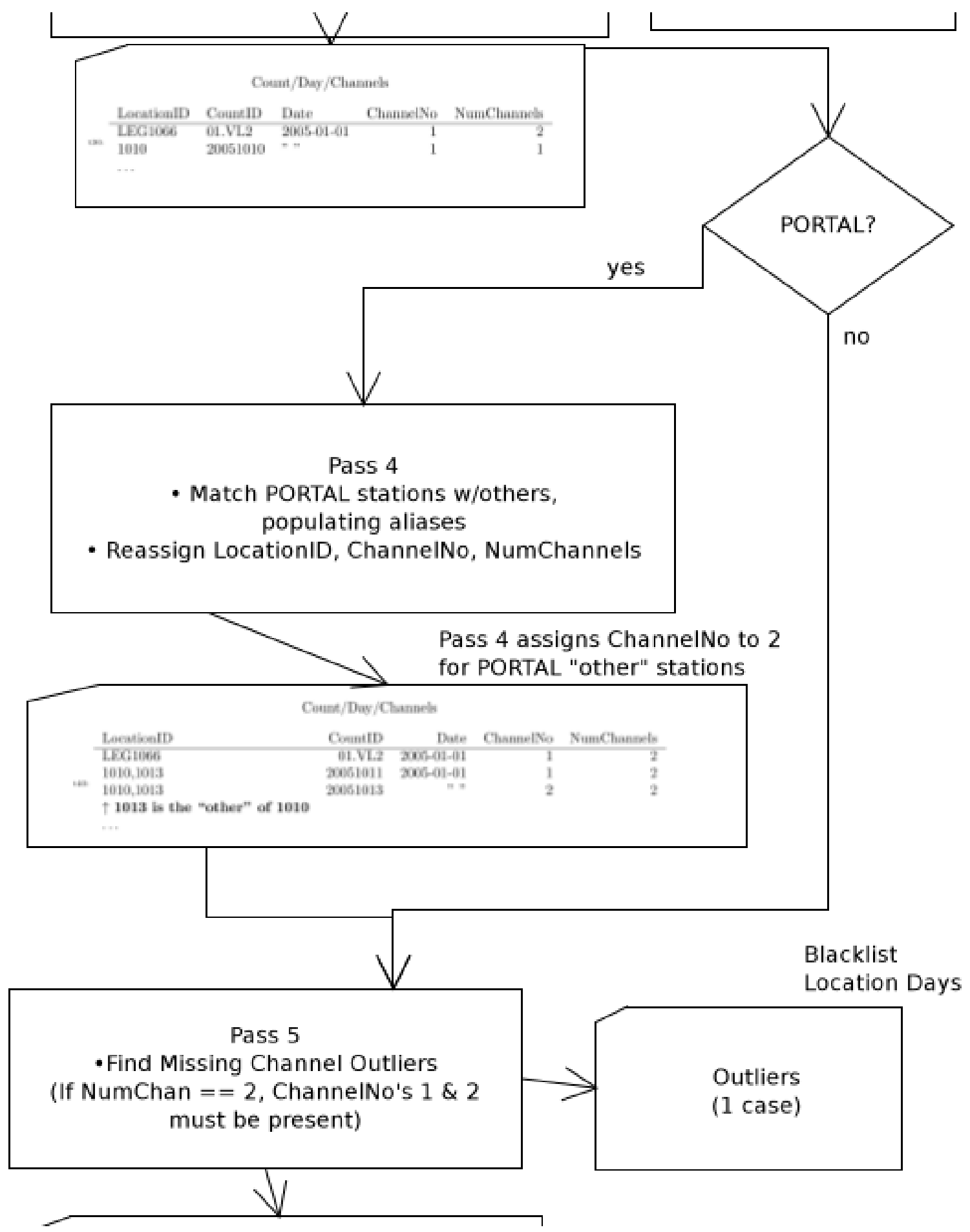

Figure A.2: TD0N pipeline, 2/4. 


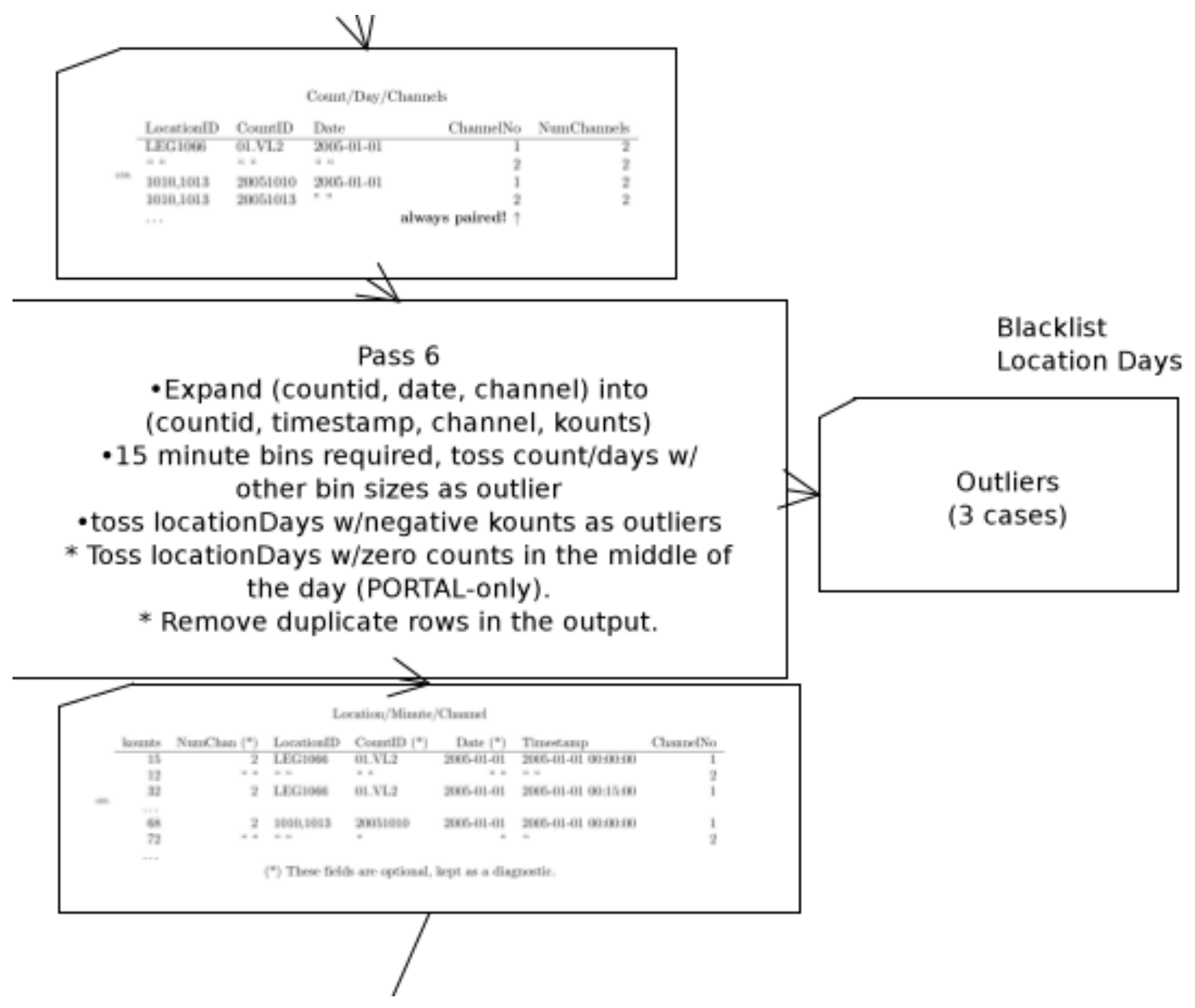

Figure A.3: TD0N pipeline, 3/4. 


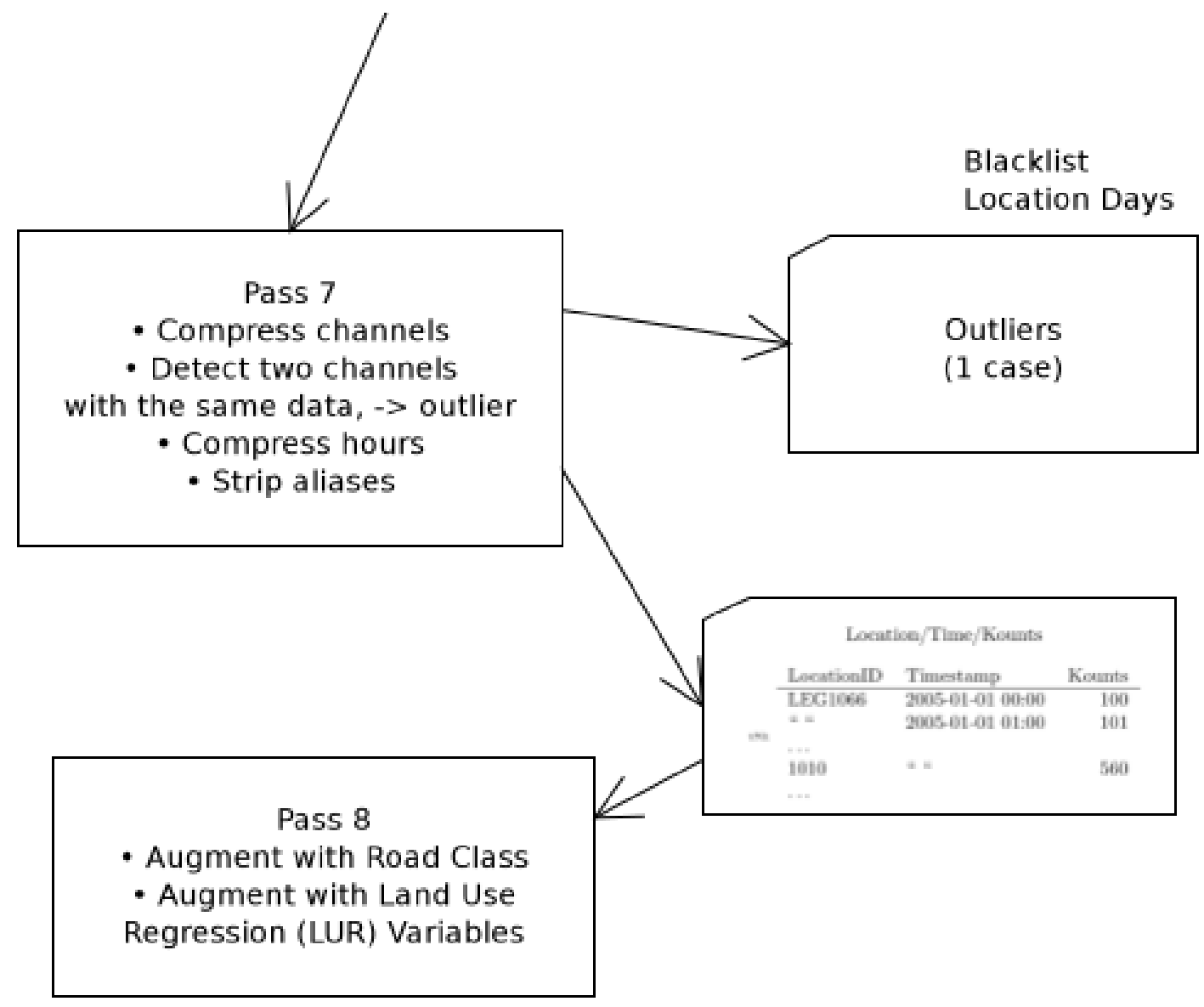

Figure A.4: TD0N pipeline, 4/4. 
Appendices

"Archives [...] include a range of format types beyond traditional paper, including [...] geographic information systems" 34, p. 3].

"You're making history." (Jeffer Daykin, Capstone teacher at PSU in 2010, on the work of an archivist)

For both collections, we created accession description forms wherein our form is based primarily on the example given in [34, p. 53], with the idea of the Restrictions field, and the idea of giving our archive a distinctive name, was borrowed from the University of Wisconsin-Parkside University Archives Accession Data form in 64 . p. 56].

To better document our activity and to allow some automatic interaction with the archive, an accession $\log [34$, p. 52, Figure 4-4] is maintained in the relational database management system (RDBMS). The log is called "data_origins". It persists in the PostgreSQL database called _8666_9078_6965_namelist.

Our work commenced with the accession of a database called TDAT. This is a collection of traffic counting records made by the city of Portland (COP), almost all made with tube counters outfitted with a pneumatic hose. For the last 8-10 years, the city has been using the JAMAR brand 1 ATRs (Tom Jensen of PBOT, private communication, 2015-09-18).

TDAT was assigned for this research the catalog number 001 and is thus often referred to in this paper as Archive 001 or just Archive 1. The principle of respect des fonds [65] requires that we carefully isolate this archive from accidental or intentional modification, yet we will be continuously augmenting and annotating the archive by, to take one example of many, marking outliers. Our approach has been

${ }^{1}$ http://jamartech.com/ accessed 2015-11-25. 


\section{A.3. ARCHIVAL PROCESS}

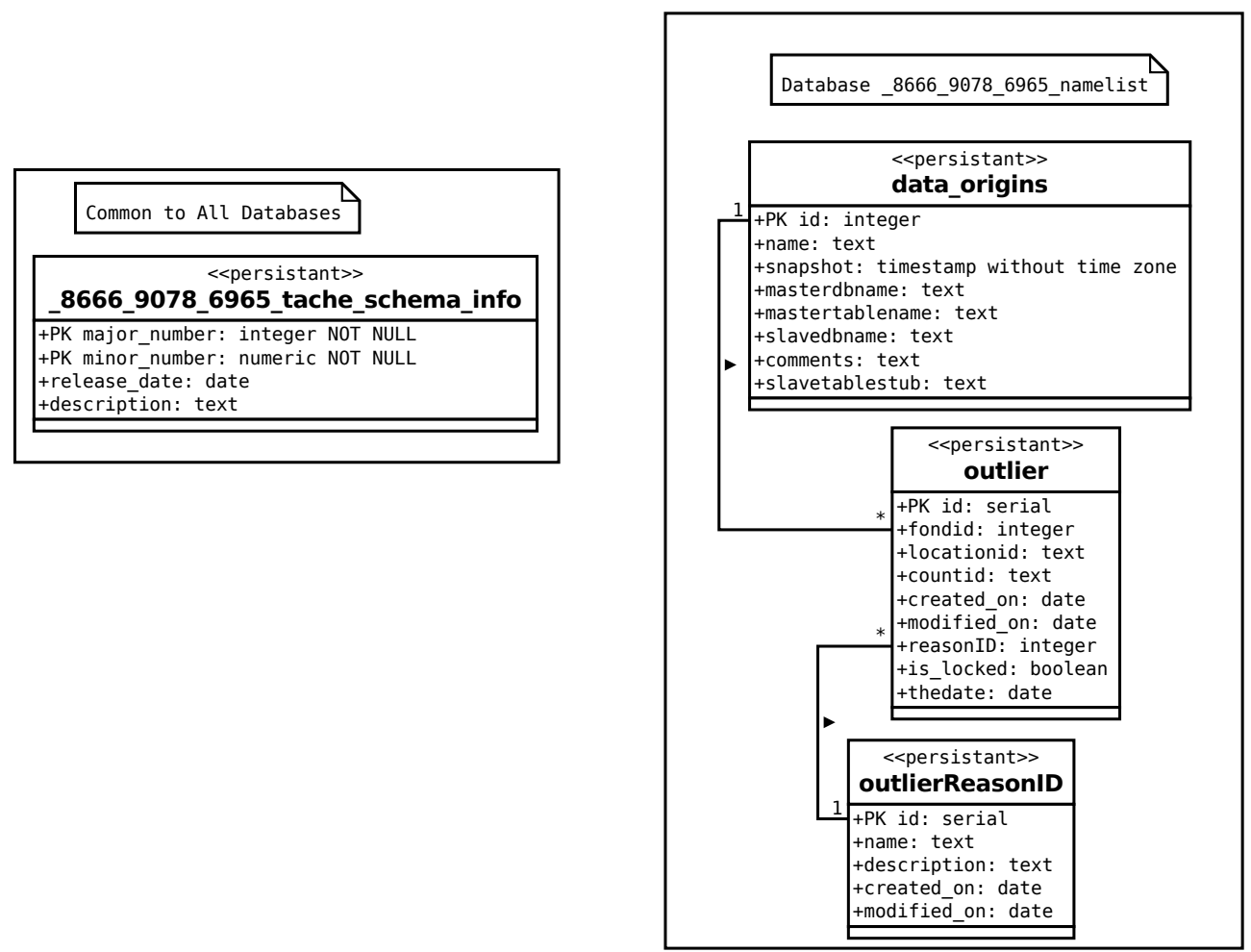

Figure A.5: A UML diagram of the namelist database. We created this finding aid to guide our manual and automated research into the archives.

to construct a number of auxiliary records that are co-resident with the archive, but clearly labeled as being foreign to the archive. That label is a sequence of three numbers emitted in sequence by a pseudo-random number generator. These numbers are 8666, 9078, 6965. These always appear together, usually as 8666_9078_6965 2 .

With our new identifier in hand, we constructed a finding aid, a catalog of the archive contents, called Database_8666_9078_6965_namelist (Figure A.5).

The accessioning process requires that a new row be added to the table called data_origins, which is unique to the entire archive (a singleton as described in [66]).

\footnotetext{
${ }^{2}$ This identifier is unique to the research described here and does not occur with import anywhere else in the entire cultural record of mankind as far as we are aware due to our random generation of these digits.
} 
If an archive is listed in the data_origins table, it is part of our archive. The ID in data_origins is the archive catalog number, e.g. $\mathrm{id}=1$ for TDAT as we described above. The name is something short and descriptive. Snapshot is a time-stamp that is attested and meaningfully descriptive of the temporal extent of the archive. Comments are free text but must include a citation for the value used in snapshot.

The remaining fields in data_origins guide our automatic analysis system to the named records in the archive where key indices and values are to be found.

The resulting archive we have dubbed the PSU Tarchive (traffic archive).

TDAT

We solicited the TDAT count database from the city Bureau of Transportation (BOT). There was dialog within the Bureau, preserved in emails, expressing concern that transferring count data at a detailed level would be an unreasonable burden on the Bureau. It was suggested, and agreed to, that the Department would give us a copy of the entire database, which we would mine for the data at high resolution.

The database was downloaded by File Transfer Protocol (FTP) in an encoded form used by a commercial firm. Our first task was to crack the code to free the data. This task was given to the Research Computing Department at PSU, who worked on a solution for some months until the decision was made to purchasing a commercial decoder. The result is a database in industry standard SQL format.

Thus did data collection and description proceed. Exploring the data progressed through a series of email exchanges with a senior technologist at PBOT, who taught us how to find and extract counts, and a method for geocoding counts.

We built confidence in the quality of the TDAT data progressively over several year of exploration and analysis. The first potential problem was corruption in the form of the full proper names of city employees and contractors in a database 


\section{A.3. ARCHIVAL PROCESS}

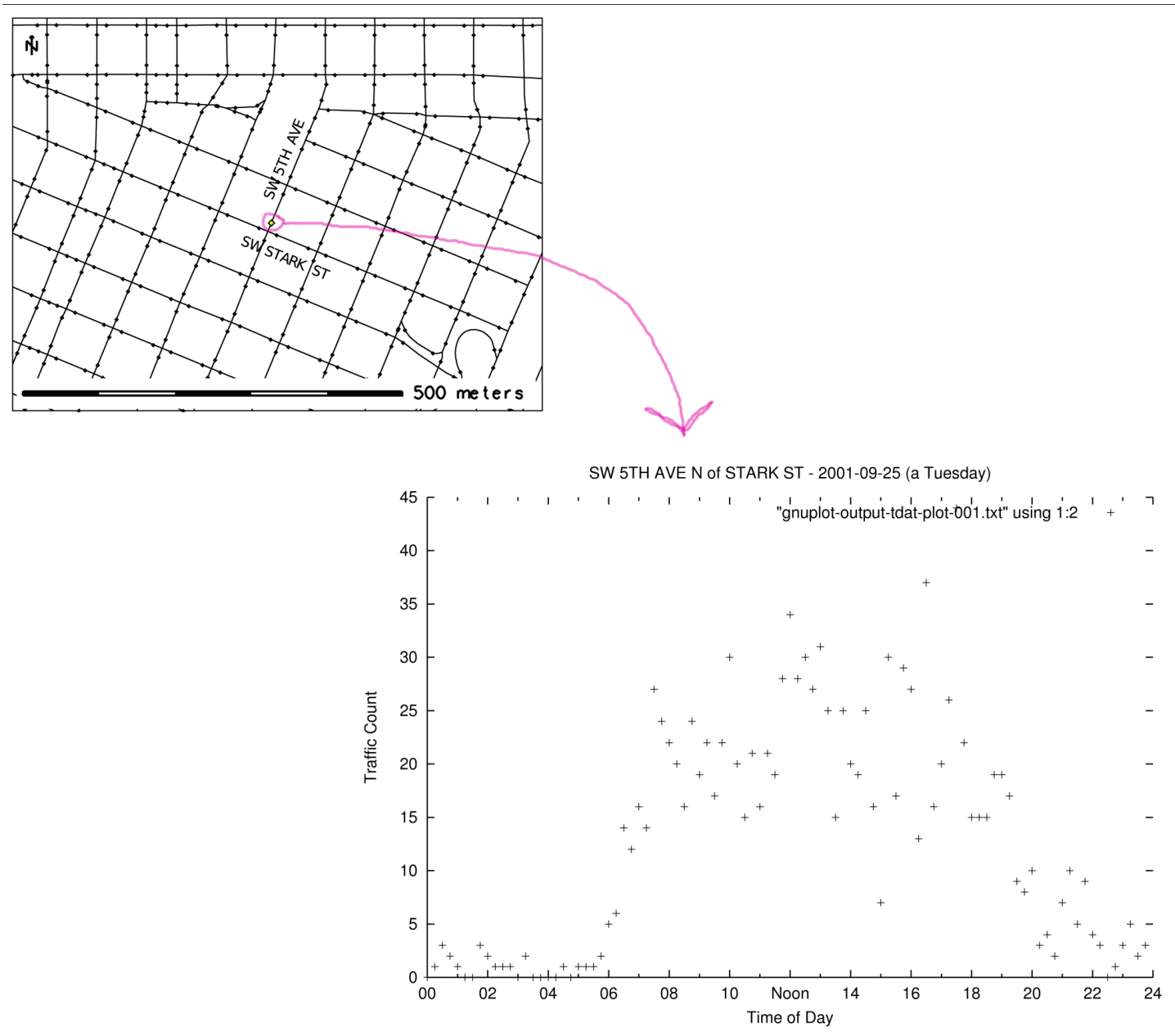

Figure A.6: A day of counting at one site in the center of the city.

slot (field) where street names are to be written. PBOT clarified that this was intentional because a location is required for each bill sent to an entity, and so when a person is the recipient, we get this.

We extracted counts for one site and graphed these in Figure A.6, and evaluated the graph for its common-sense scale and diurnal pattern.

We spent some time evaluating the structure of the counts. We produced the map of Figure A.7, a useful basic overview of the data we had obtained.

We obtained a GIS shapefile from RLIS which classifies the roads in a 32-factor 


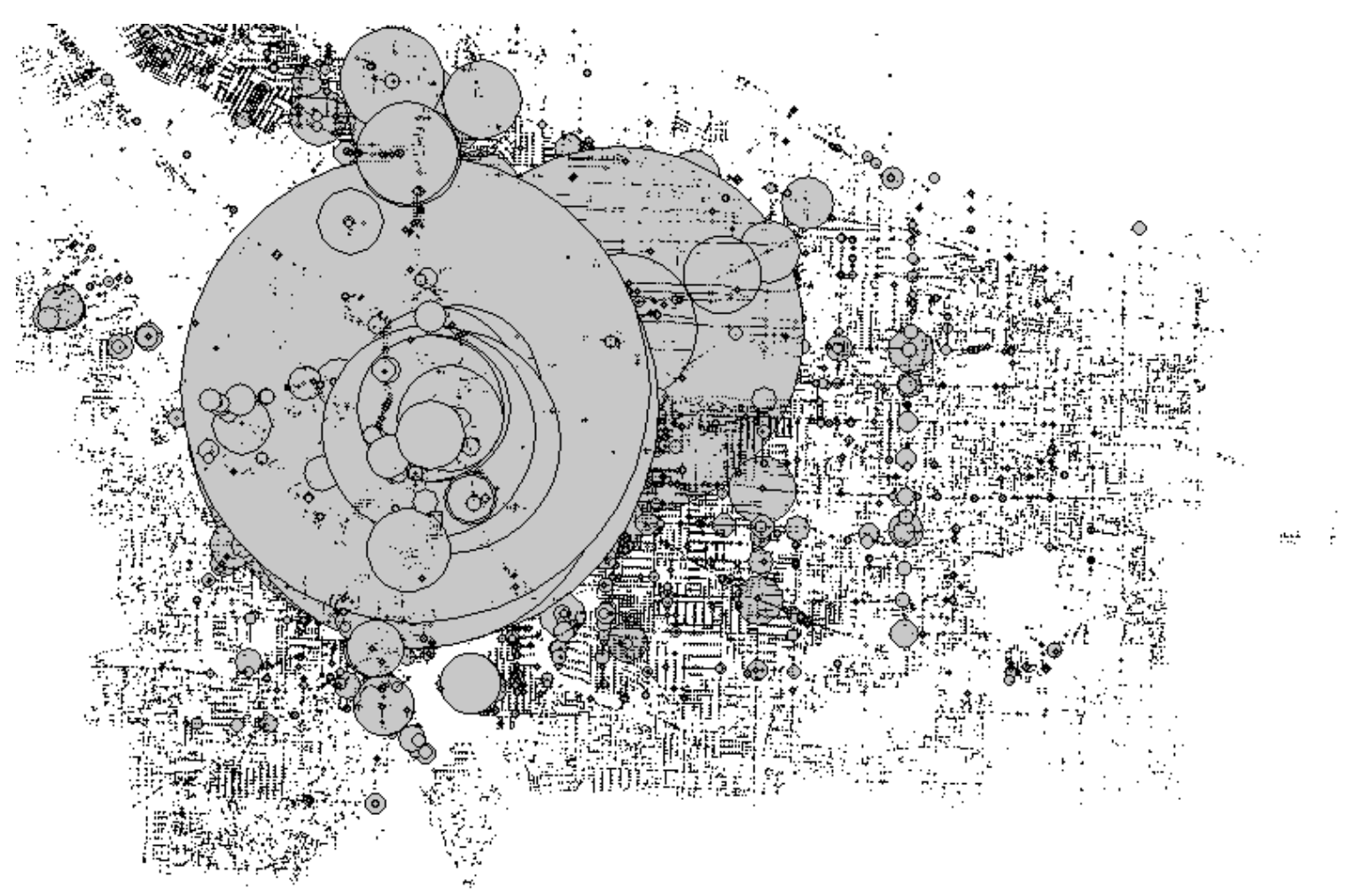

Figure A.7: Map of circles proportional to the number of times PBOT decided to open a count at the site. Note the big circles all focused on the downtown area and linear patterns of counts opened along major N/S and E/W corridors. North is up. The Willamette river can be seen as a lacuna opening in the top-left of the map. A large forested park is immediately to the west of the river at the top-left, represented as another lacuna. The remaining empty space represents the Columbia river to the north, and other jurisdictional boundaries to the east, south, and west. 


\section{A.3. ARCHIVAL PROCESS}

\begin{tabular}{lll}
\hline type & color & \\
\hline 1110 & red & Freeway \\
1121 & red & On-ramp (only) \\
1122 & black & Off-ramp (only) \\
1123 & violet & On- and off-ramp (combination) \\
1200 & blue & Highway \\
1221 & purple & On ramp (only) to highway (in the area \\
& & maintained by the City of Portland only) \\
1222 & red & Off ramp (only) to highway (in the area \\
& & maintained by the City of Portland only) \\
1300 & indigo & Primary arterial \\
1400 & green & Secondary arterial \\
1450 & red & Other arterial \\
1500 & cyan & Minor streets \\
1521 & yellow & Local street to local street connector \\
& & (Portland only, subarea = 'P'). \\
1700 & magenta & Private named road; private right-of-way exists \\
1800 & gray & Unnamed driveway; private right-of-way exists \\
2000 & brown & Unimproved road, passable by emergency vehicles \\
& & (e-911) only (in the area maintained by the City of Portland only) \\
5301 & red & Primary arterial with rapid transit \\
5401 & orange & Secondary with rapid transit \\
5501 & aqua & Minor with rapid transit \\
\hline
\end{tabular}

Table A.1: Represented road classes in the toy study area, with color codes assigned.

scheme (freeway, primary arterial, minor streets, \&t). We defined a toy study area to practice on, and constructed Table A.1 to produce the map of

We built competence in mining the TDAT data through constant probing, reshuffling, comparing the results of a number of ad-hoc analyses that we conducted, punctuated with the formulation of questions for our domain experts at PBOT, who have always replied courteously and promptly 3

Much of the complexity of mining the TDAT database is the result of the almost completely inverse structure of the TDAT RDBMS when compared to PORTAL. Where the latter is flat and simple making the addition of a new record just a single row in a table, TDAT involves adding several interlinked records in several

\footnotetext{
${ }^{3}$ Portland, OR has a tradition of strong civic involvement.
} 
tables. Where PORTAL's counters are automatic, often neglected, and permanent installations, TDAT's counters are carefully hand-placed, watched over, and highly temporary. This leads to a great variety of corner cases which we shall document here.

Accessioning the PORTAL process involved molding the PORTAL data dictionary to closely resemble the TDAT data dictionary. Consequently, we appropriate some terminology from TDAT. A count happens when someone decides, for any reason, to count vehicular traffic on a road. When this happens a unique (within the archive) count ID is generated and assigned to the count. For PORTAL, each station/day is assigned a unique count ID by combining the station ID and the day. A kount is a recording of vehicles that actually passed by in a given time period. A kount is always an exact integer.

We explored the published data and metadata thoroughly, and interacted via email with the PORTAL staff to obtain the information needed for our research. We learned that PORTAL has recorded traffic counts at 15 minute intervals on all of the area's freeways and highways for more than nine years.

\section{The curated PORTAL data at RDE}

We first obtained a curated and well-documented extract of the PORTAL data from the Research Data Exchange (RDE), (https://www.its-rde.net accessed 2014-03-20). The RDE is a US Federal Government-held database which hosts curated and documented data sets. The PSU PORTAL group put considerable time and effort into delivering a fine extract from their raw data, cleaned and documented. While the set is too small (just two months of coverage) to be useful for our project, we found this to be a useful starting point for learning how to clean 


\section{A.3. ARCHIVAL PROCESS}

and reduce the highway and freeway data.

We sought for and obtained confirmation (K. Tufte, private email) that the RDE data set, which is identified by the name "Test Data Sets from Portland OR" with a publication date of 2012-04-16 2016-10-27) is identical to the data set known as "PORTAL FHWA" which is available from (https://portal.its.pdx.edu/fhwa accessed 2016-10-27).

We were able to use this valuable resource to develop our analysis while we continued to dialog with the PORTAL group about an extract of the raw data. Kristin Tufte was the principal on the PORTAL RDE (aka FHWA) data set preparation; we are not aware of the names of any other contributing parties.

In the process we built confidence in our tools and in the appropriateness of using the PORTAL data together with TDAT.

\section{Mastering the PORTAL raw data}

With the help of the PSU Research Experience for Undergrads (REU) program, who lent us Chad Sarni for two days to operate the PORTAL web site, we obtained a snapshot of the entirety of the raw count data (starting on 2005-01-01 and ending on 2014-07-27). With some searching, we obtained a complete map of the sites were counting was done.

We learned that PORTAL has both freeways and highways in it (and TDAT has highways but no freeways). The total list of freeways and highways in PORTAL is given in Table A.2. We concluded that the following roads are highways: OR 217, SR 500, US 26, SR 14 and we classified them as such for the research, leaving the rest of the roads in Table A.2 classified as freeways.

All of our GIS work was conducted using data projected into the Lambert Conformal Conic (LCC) projection, with ellipsoid grs80, selected because this is the default of a large curated base layer archive (RLIS) maintained by the regional 


\begin{tabular}{l} 
freeway name \\
\hline I-84 \\
I-5 \\
I-405 \\
I-205 \\
WA I-5 \\
WA I-205
\end{tabular}

Table A.2: Portal freeway coverage.

authority (Portland Metro). The freeway and highway recording station positions published by PORTAL are in lat/long coordinates. To obtain a GIS layer of the PORTAL station locations, we began with the lat/long station positions in the station list posted as part of the RDE curated set (Section 2.3.2). We required an LCC projection of these lat/long station positions for compatibility with the RLIS street map.

We used cs2cs version 4.8.0, the "cartographic coordinate system filter" (cs2cs documentation) by Frank Warmerdam and Gerald Evenden ${ }^{4}$ to translate station positions from lat/long into LCC for compatibility with our RLIS street map.

The first step in understanding the complex TDAT database was to construct a finding aid in the form of a data dictionary. With the database in PostgreSQL after the translation work of Section 2.3.1, we were able to use the LibreOffice tool to quickly create the overview present in Figures A.8 and A.9

Q \& A sessions with our PBOT experts proceeded through three distinct phases: ${ }^{4}$ https://github.com/OSGeo/proj.4/wiki accessed 2016-10-27. 


\section{A.4. DATA CLEANING DETAILS}

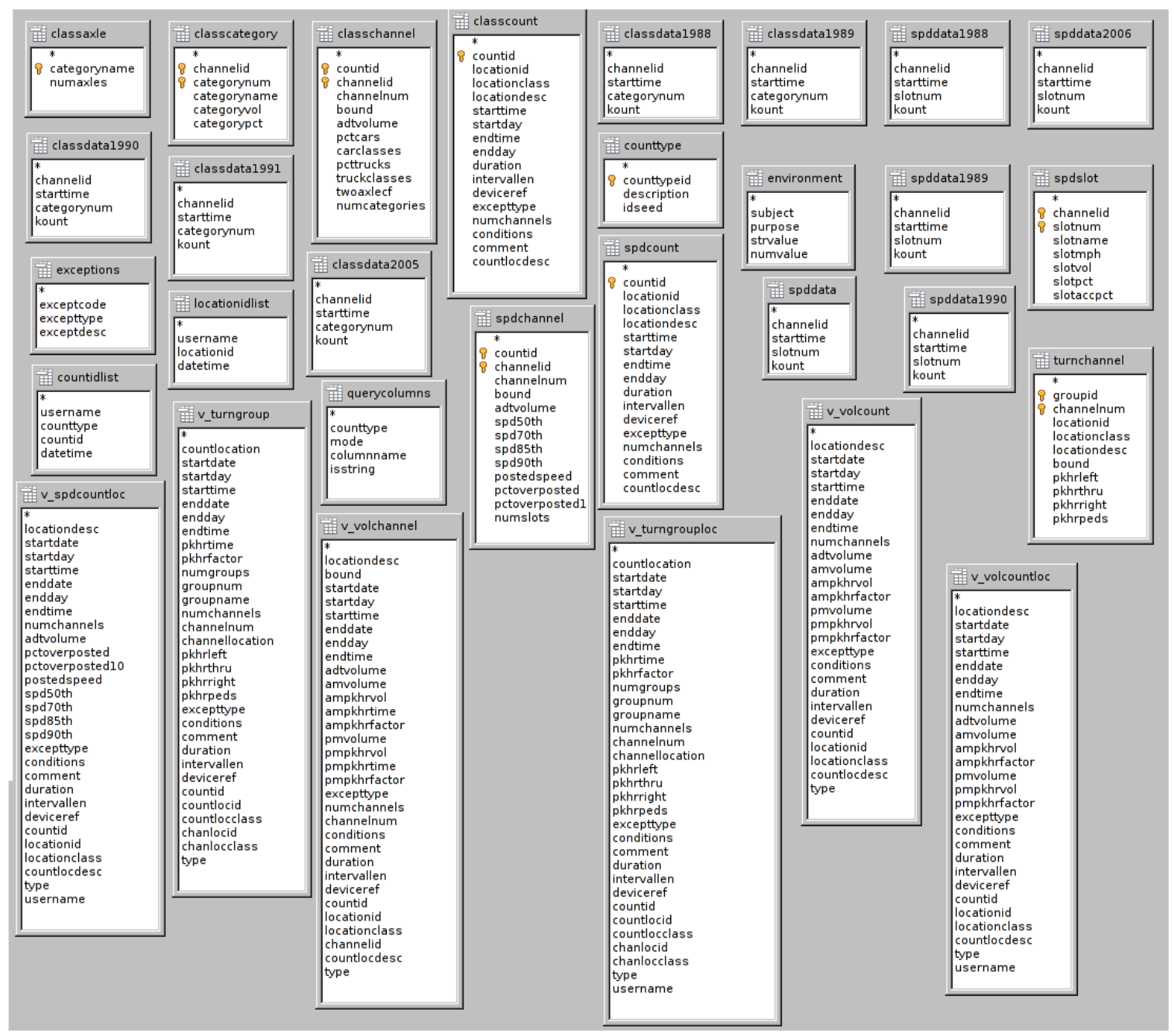

Figure A.8: The TDAT schema diagram, part 1. 


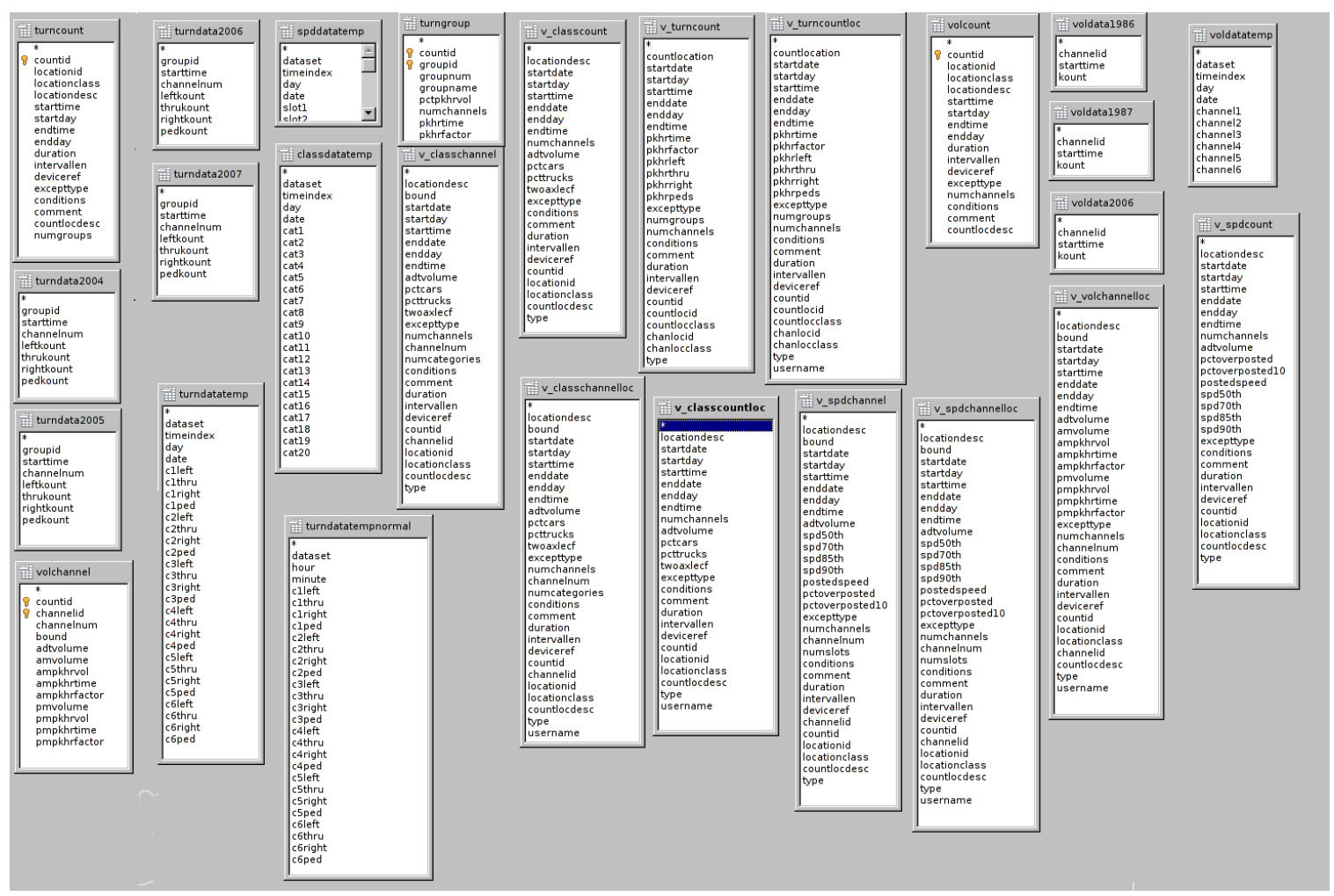

Figure A.9: The TDAT schema diagram, part 2. 


\section{A.5. TDAT ROAD CLASS ASSIGNMENT - TECHNICAL DETAILS}

1. Obtain believable, accurate counts for a given time and place.

2. Find the road functional class belonging to a count.

3. Surety that all counts that belong to a site are discovered and counted in data reduction.

Each of these phases brought new confidence in the quality of the data, although the process was not without bumps. Two in particular had us concerned. The first was the inclusion of people's names as detailed in Section 2.3.1. The second was a failure of the road class assignment of Phase 2 to properly assign a smattering of sites.

The first was explained easily as we said. The second spurred us on to a careful analysis, in early 2014, of road class assignment which we shall now discuss in some detail.

Road class in TDAT is complex. The Bureau of Transportation does not consider road functional class to have any place in the database of traffic counts: "we presently have no plans to put the federal classifications in our location model" (Jamie Throckmorton of PBOT, private communication). We identified a source of road class information provided by the regional planning authority (Metro). Identifying count sites in the BOT archive, which are point data in a GIS layer called segments, with roads in the Metro road map (RLIS streets) was a major GIS effort, but we are satisfied today that we have succeeded with this work.

The count sites are geocoded and assigned to one segment of the city's road network. The count sites are filed under the name nodeleg, which is a shapefile containing vertex geometry. The vertex attributes connect each vertex to a line 
Appendices

segment in the street map, which is called segments. Segments is another shapefile, containing line geometry. The attributes of segments identify the street name.

We consider compatibility of our system with EPA MOVES a priority, so we read [67] to find that MOVES requires road types "based on the HPMS classification" (ibid, p. 28). We obtained and compared a variety of candidates (ODOT, PBOT SDE, Topologically Integrated Geographic Encoding and Referencing (TIGER), HPMS, and RLIS streets).

We concluded by selecting RLIS streets, whose road functional class "codes are specific to our region" (Kelly Hauger, GIS specialist at Metro, private email), based on advice from an expert at PBOT. RLIS streets is a GIS shapefile curated by Portland's regional authority, part of the RLIS archive. It contains line geometry. The attributes identify the street name and the road class. We had to find a way to impute the road class from streets to segments.

Our effort to use a spatial join fails due to great disparities $\S$ A.5.1

between road maps

Our PBOT expert suggested a spatial join. If both maps are accurate, we reasoned, it would be quite easy to accomplish a spatial join, effectively copying the road class into segments. We had to proceed carefully, because the accuracy and usefulness of the model is a function of the accuracy and usefulness of the road functional class assignment. Thus, we spent time planning this step, exploring systems of road functional classification and alternative data sources for road class in our study area.

Our data sources selected, we attempted the spatial join. The join failed because of pervasive differences between the two vector layers (RLIS streets and TDAT segments). The two layers are evidently based on a common source put together perhaps in the 1970s, which have diverged enormously since that time. Another 


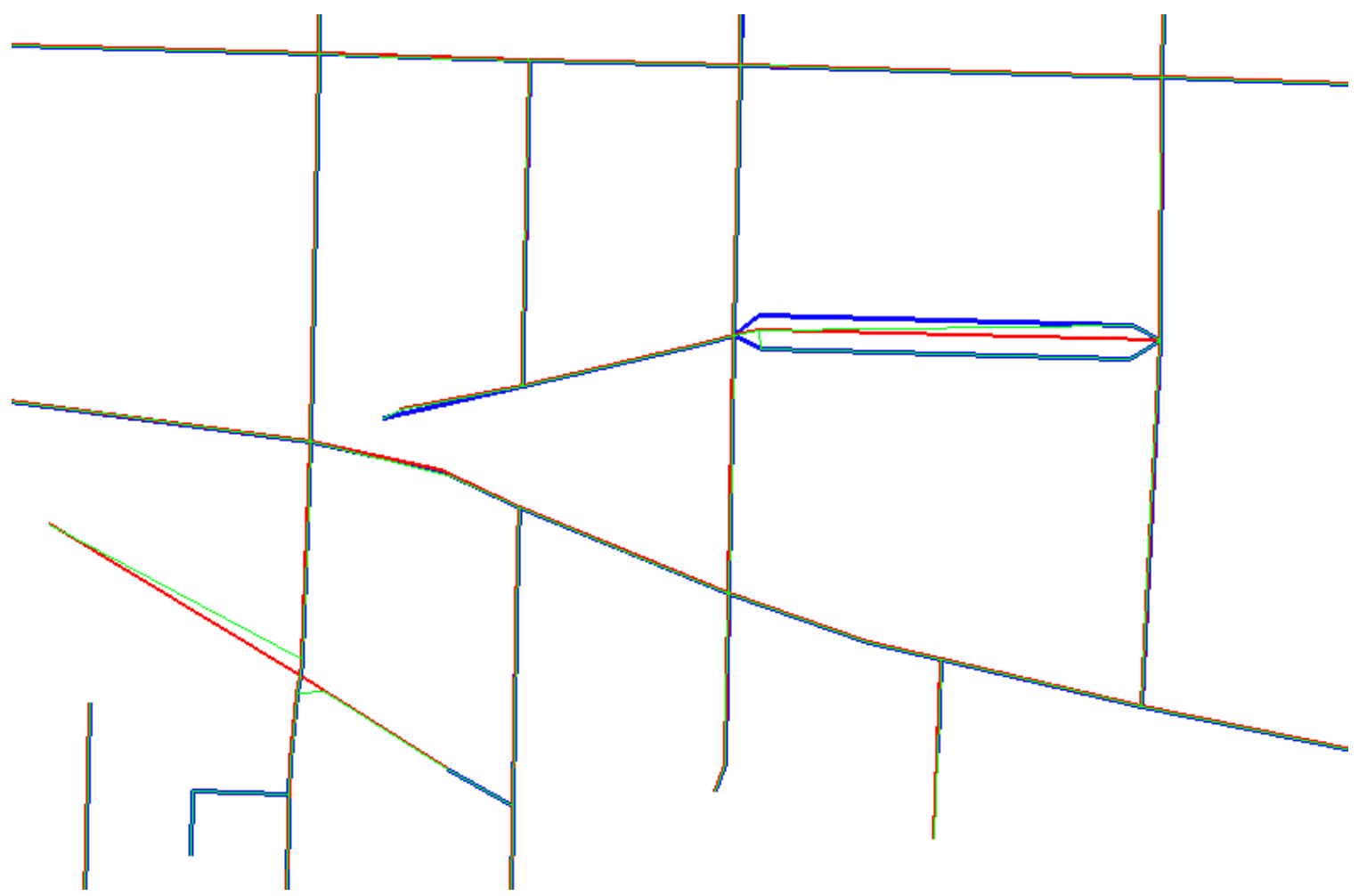

Figure A.10: An overlay map of the area around SE 29th and SE Waverleigh. North is up. Red lines are road segments from the PBOT segments. Blue lines are road segments from RLIS streets. Green lines represent the software's best attempt to spatially join RLIS streets with PBOT segments. Note the remarkable degree of divergence between the two maps, far too great for an automated system to reconcile them.

approach was needed.

One example of several we examined of the complete failure of an automated spatial join based just on the linear street segment geometry is given in Figure A.10. This type of divergence between the maps is pervasive, reflecting in the author's opinion decades of customization by two very different governmental units.

\section{$\S$ A.5.2 \\ A key is found that enables road class assignment}

A single key identifies - often, but not always - a road segment in both layers. In TDAT the key is the last six digits of the parent ID returned by

psql -x -d rlis-gis -c "select * from locheir 
where LocationID = 'LEG66360' and

systemid = 'STREET';"

. That select returns

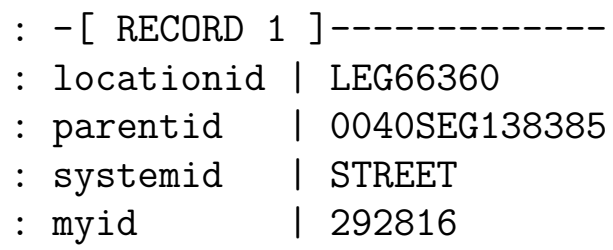

The value 138385 matches the local ID in RLIS streets, as in

psql - $\mathrm{x}-\mathrm{d}$ rlis-gis -c "select * from segstr_clean

where localid = 138385;"

, which returns

\begin{tabular}{l|l} 
- [ RECORD & 1 ] \\
cat & 79928 \\
comments & \\
direction & \\
fromint & \\
ftype & AVE \\
lcity & PORT \\
lcounty & MULT \\
leftadd1 & 300 \\
leftadd2 & 398 \\
leftzip & 97204 \\
length & 262.5003299528 \\
linkpath & \\
localid & 138385 \\
location & \\
modifiedby & \\
modifiedon & \\
modifiedus & \\
prefix & SW \\
rcity & PORT \\
rcounty & MULT
\end{tabular}


\begin{tabular}{l|l} 
rgtadd 1 & 301
\end{tabular}

\begin{tabular}{l|l} 
rgtadd2 & 399
\end{tabular}

rightzip | 97204

shape_leng I

streetname | $5 \mathrm{TH}$

toint $\quad$ ।

type $\quad 5501$

zero $\quad \mid 0$

The spatial join indicated how tenuous this link is (the two maps diverged significantly over time), and in a flyover we noticed an anomaly: highway-typed streets deep in a residential neighborhood. These mistyped streets were a serious concern, so we examined this case in particular and found that our analysis is unaffected because no traffic counting ever happened at these particular sites.

The flag was raised however, and to regain confidence in the road class assignment we tested and proved the theory that every counter site in TDAT where counts actually happened is sited within a short distance $(4 \mathrm{~m})$ of a road segment in RLIS streets which agrees with the road class we previously found for the site by the tenuous link of Appendix A.5.2.

We found that $1.7 \%$ of the sites do not have a road on the authoritative map (RLIS streets) with the appropriate road functional class within four meters of the count site. We are unable to say anything definite about these sites without ground truthing them, except that the remainder of the analysis came so close to perfectly matching road class via the tenuous link and road class by the type of spatial matching assigned here that they are most probably correctly assigned. Divergence in the location and dimensions in the road segments given in the two maps is great, as we have demonstrated, so it is expected that our careful analysis here, with its tight $4 \mathrm{~m}$ radius requirement for a match, will not always find a match 
even when the assignment is good.

At $98.7 \%$ of the sites where a count actually happened, only one type of road is found. In the other $1.3 \%$ of sites, there are more than one road classes represented in the $4 \mathrm{~m}$ circle, but the "correct" road class is always among them. We conclude that it is highly likely that $100 \%$ of the sites where counts actually happened have been assigned the correct road class. This is not a given, because our method relies on a tenuous putative link between TDAT segments and RLISstreets, two street maps that seem to have shared a common ancestor a long time ago, but which have diverged quite thoroughly since that time (for example, many or most road segments have drifted, and many road segments have been added or deleted, to better fit the needs of the individual bureaus, which are COP PBOT and Oregon Metro - a regional planning organization).

One of the vague cases is given in Figure A.11. Due to the divergence in the segments and streets maps, either of the two roads shown could apply their road class to the site indicated by the yellow cross. The geocentric coordinate system (GCS) coordinates in RLIS streets projection are given.

This $\mathrm{RC}$ validation work is epitomized by the occurrence of the text typesin as in \#if (DIAGNOSTIC_TYPESIN)

$\ln <<"$,rc,typesin_4_m_01, ... \#endif 


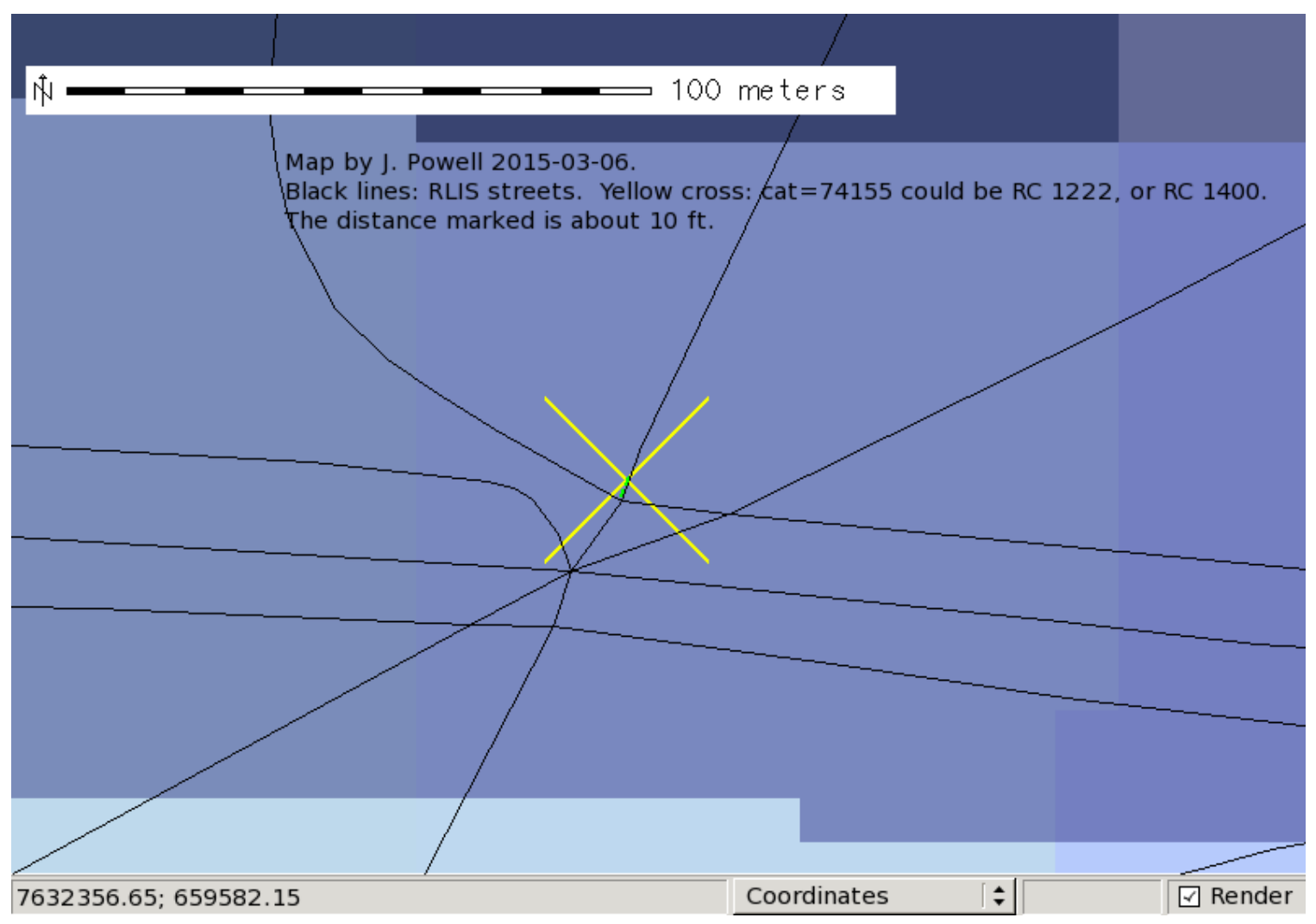

Figure A.11: Map showing one of the TDAT count sites where it is not possible without ground truthing to identify the road class of the site. RLIS streets includes two roads with different road classes in the immediate vicinity. 
Appendices

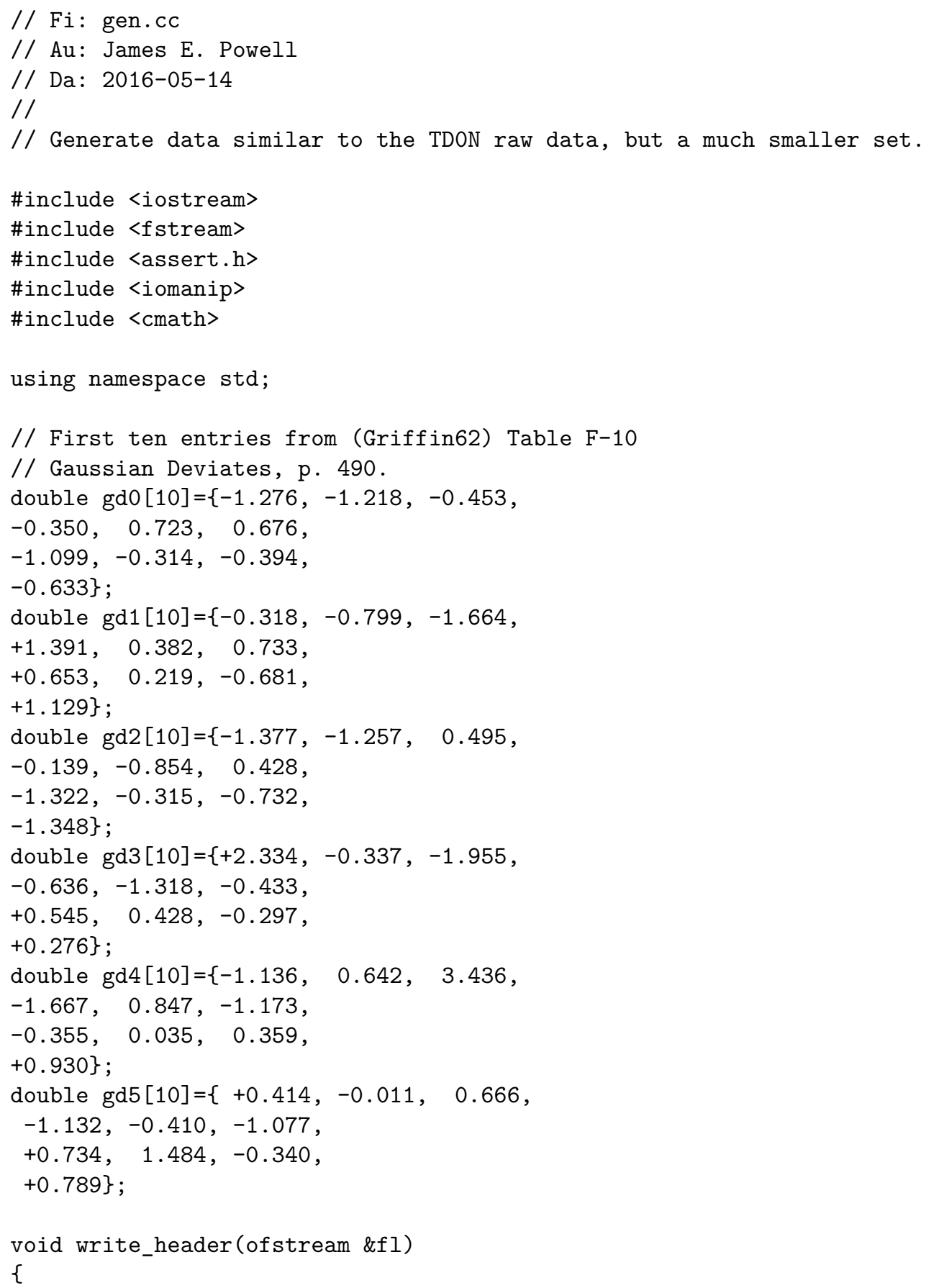




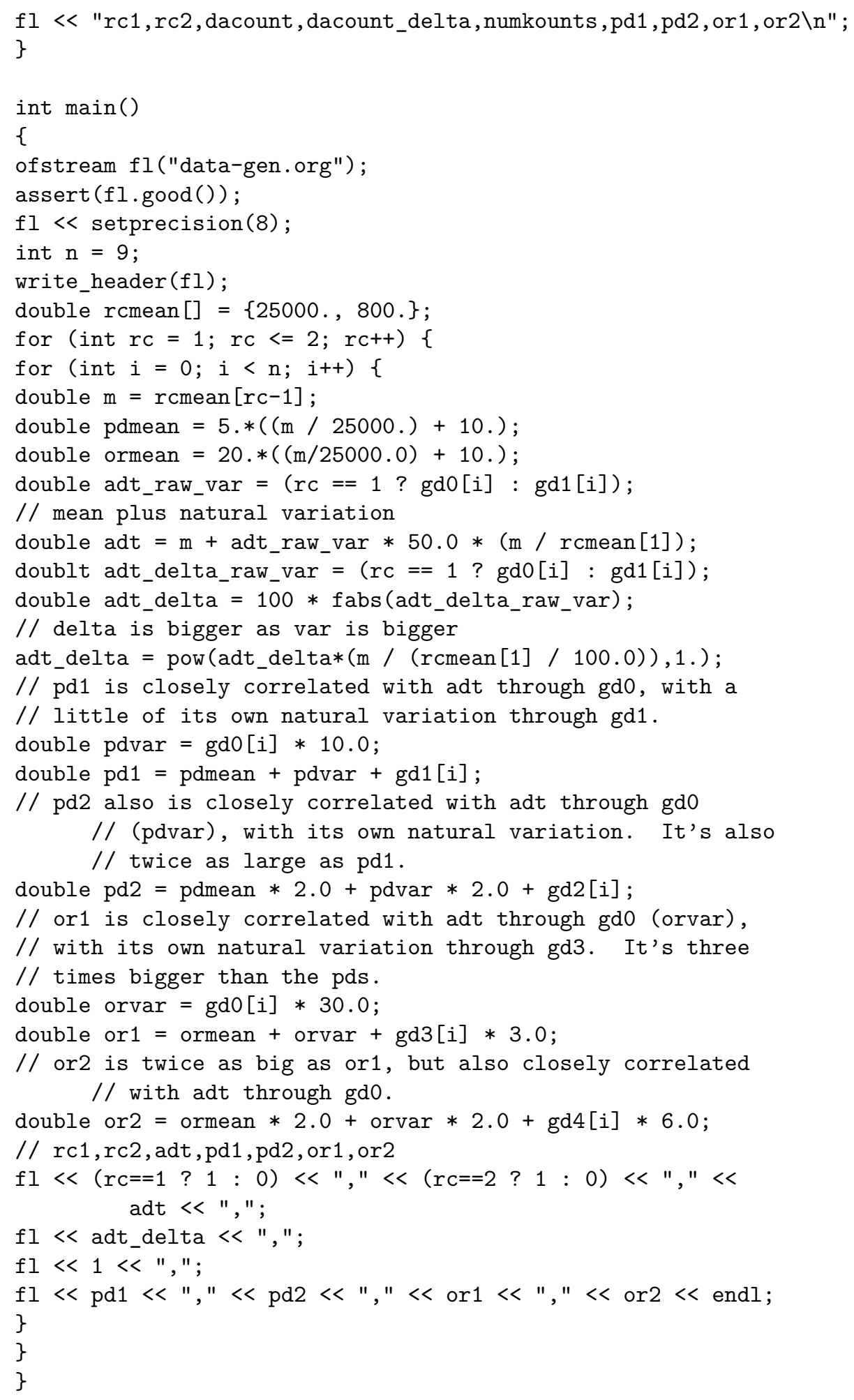


Appendices

$\S$ A.6.2

Adapt PORTAL to the TDAT data structure

We had a need for PORTAL data to appear, to the SQL interface, much like the much more hierarchically organized TDAT data. We accomplished this by creating the materialized views, using the SQL given here.

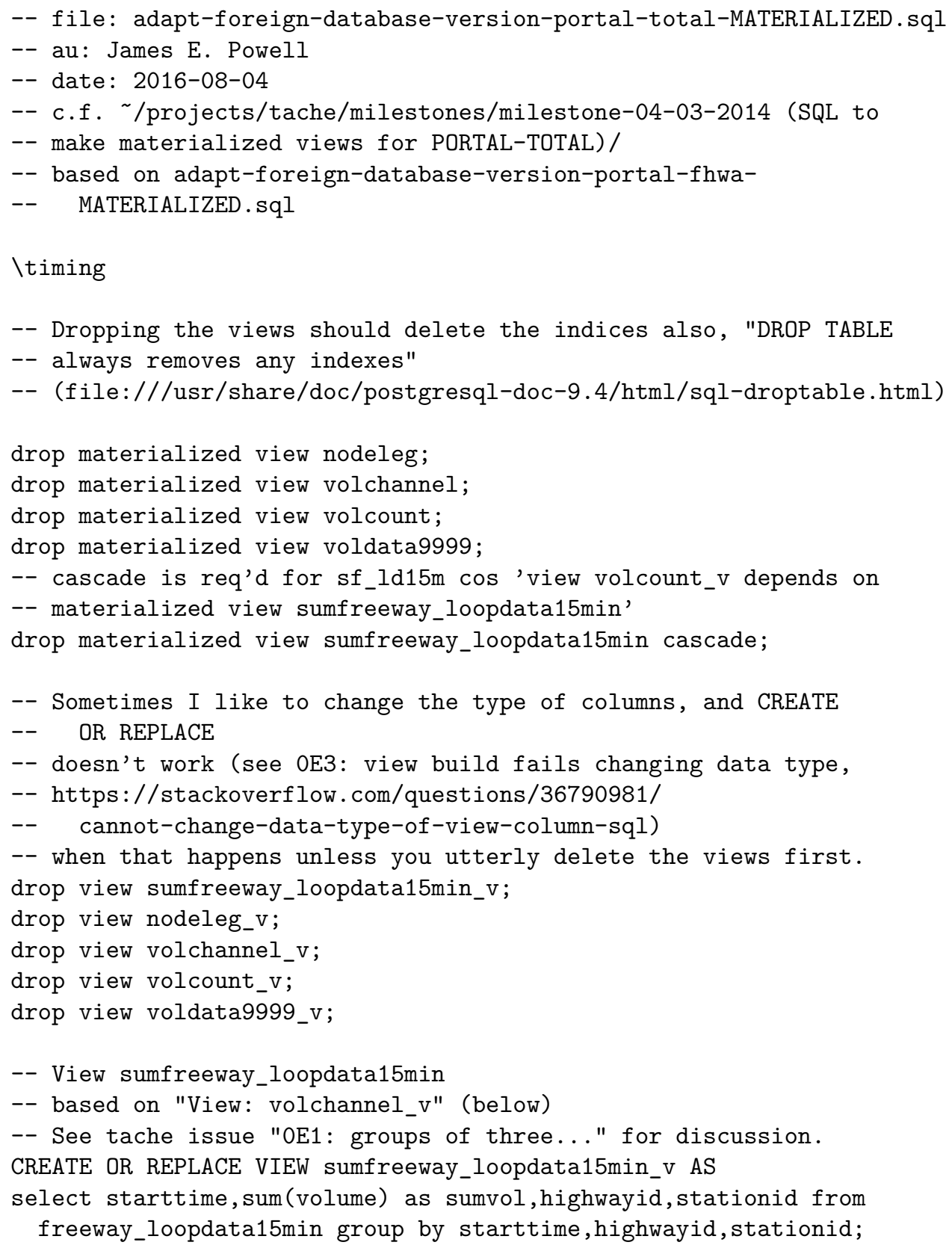




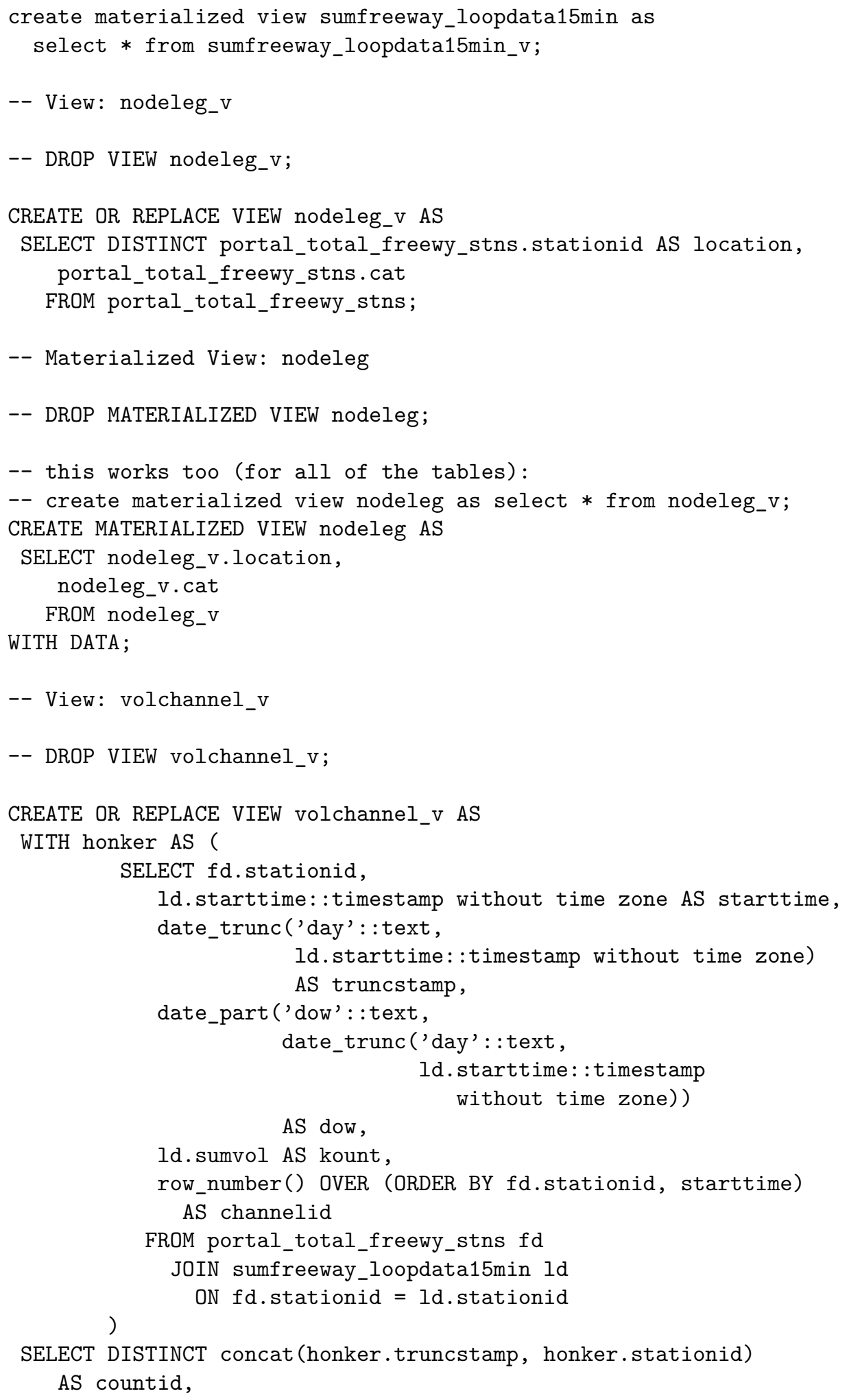




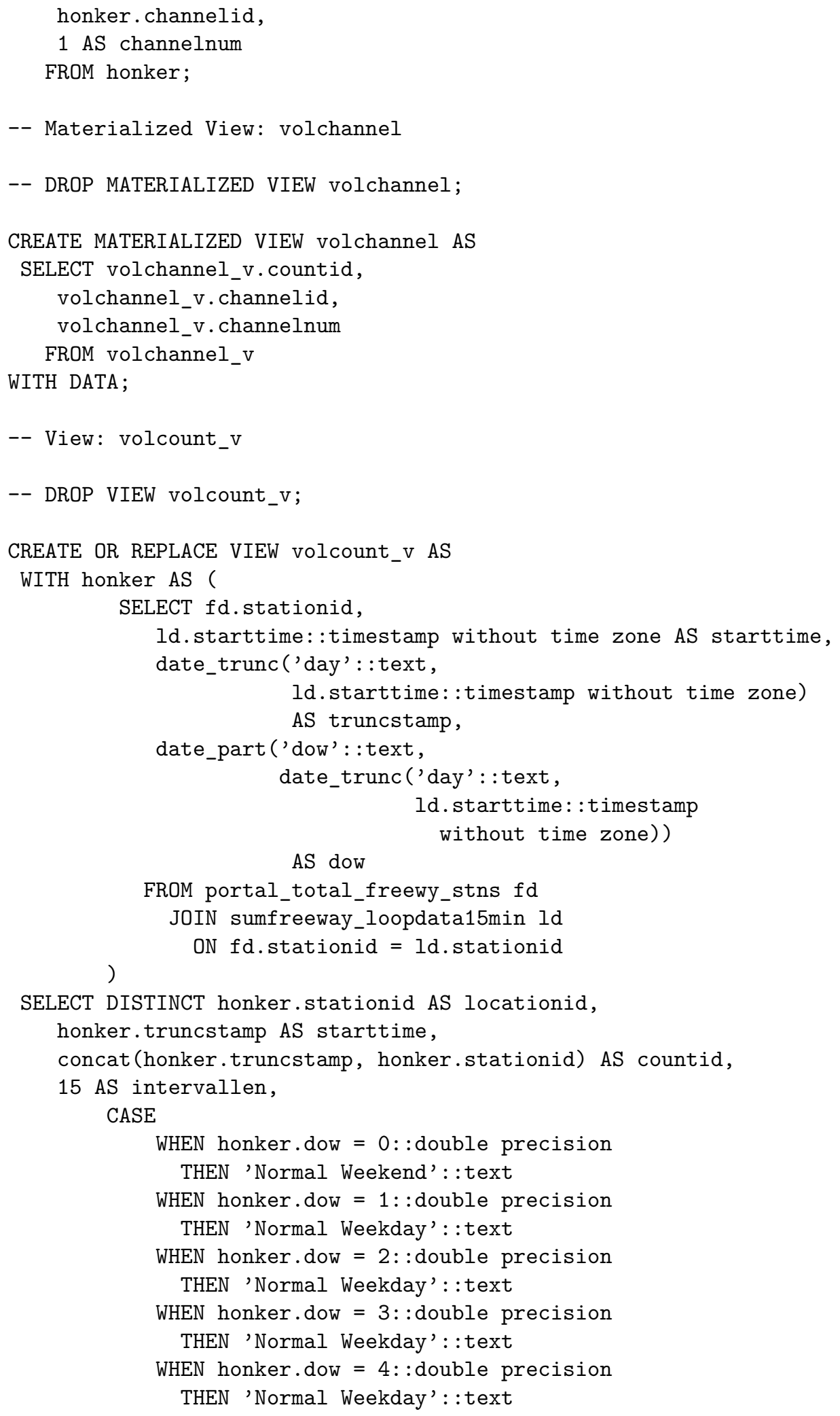




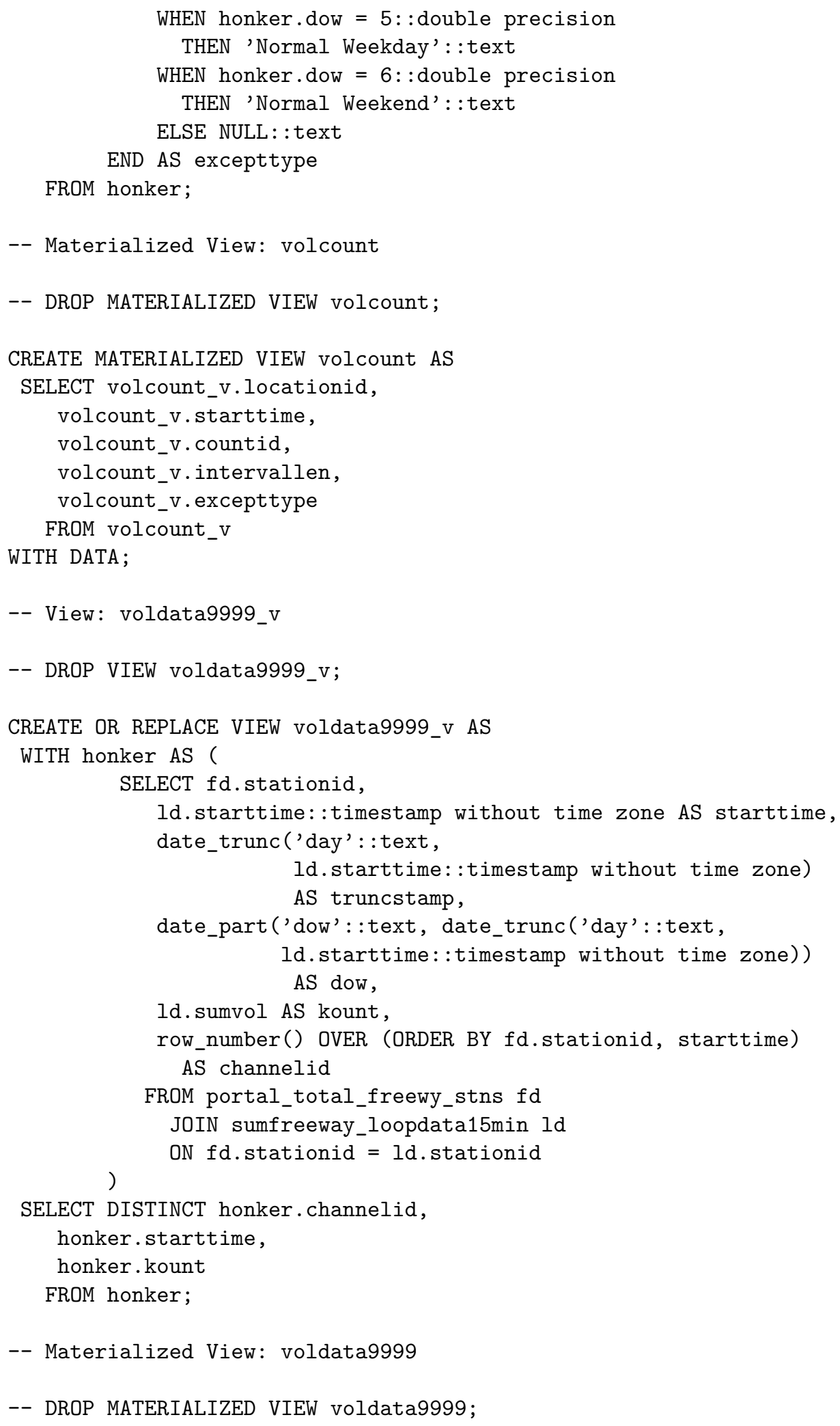




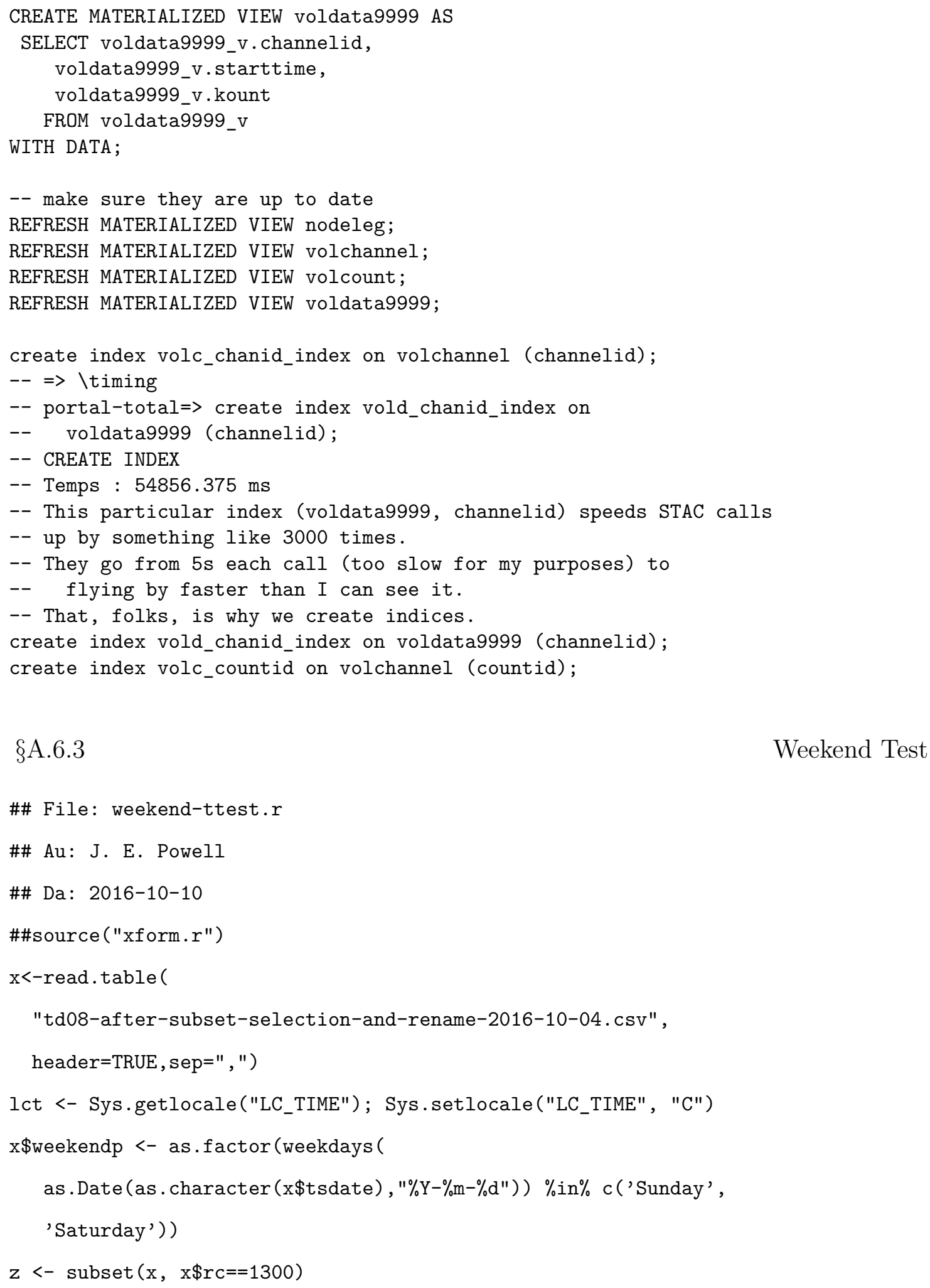

$\S A .6 .3$

Weekend Test

\#\# File: weekend-ttest.r

\#\# Au: J. E. Powell

\#\# Da: 2016-10-10

\#\#source("xform.r")

$\mathrm{x}<-$ read.table (

"td08-after-subset-selection-and-rename-2016-10-04.csv", 


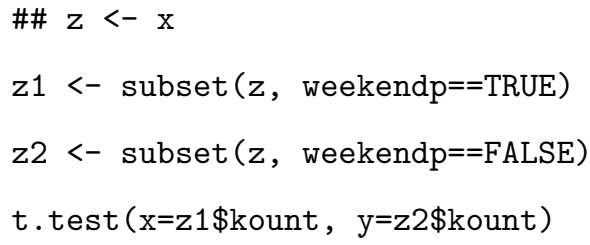

Diagnostic Plots for the Log Transformation.

\section{$\S A .6 .4$}

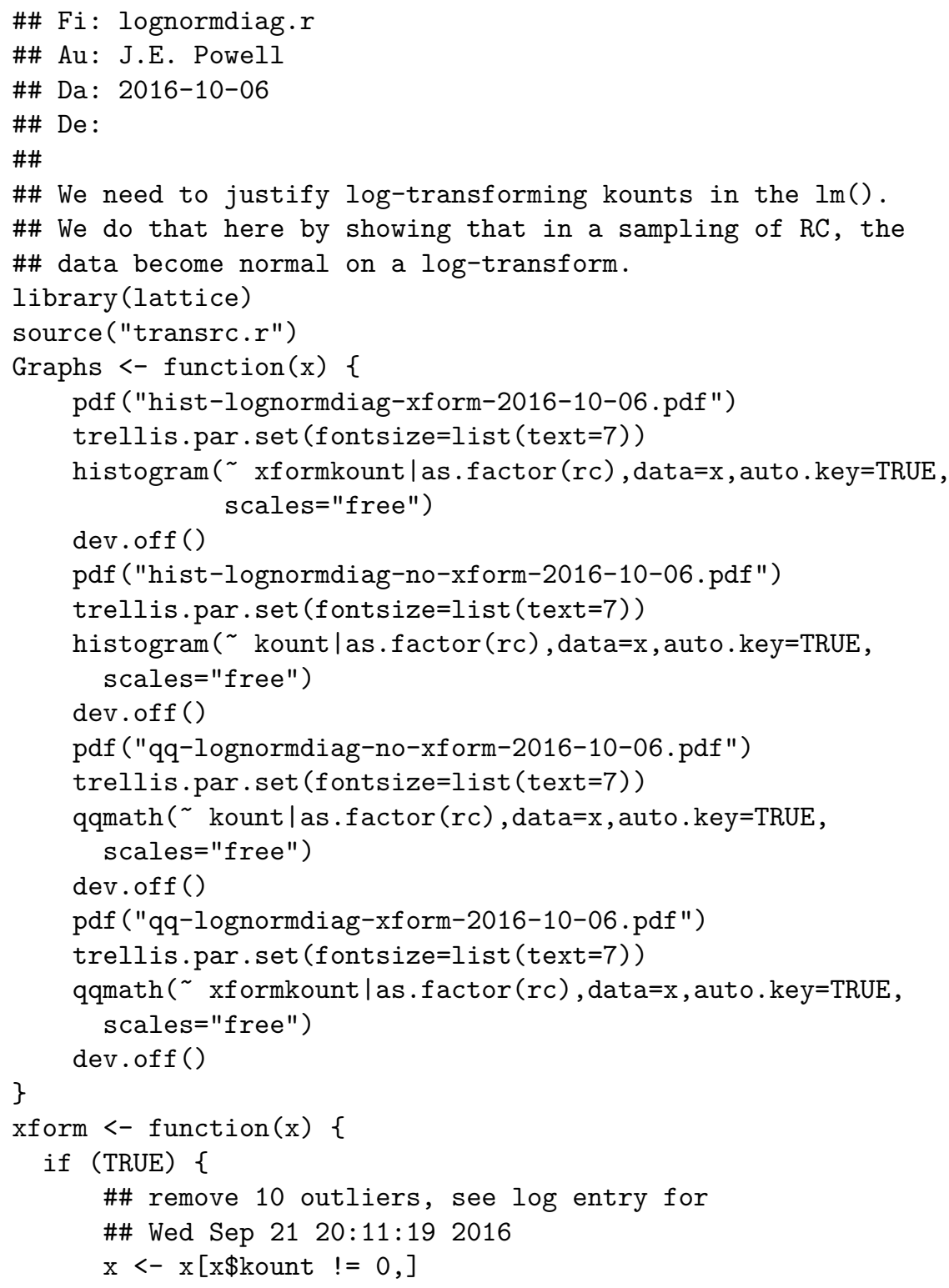




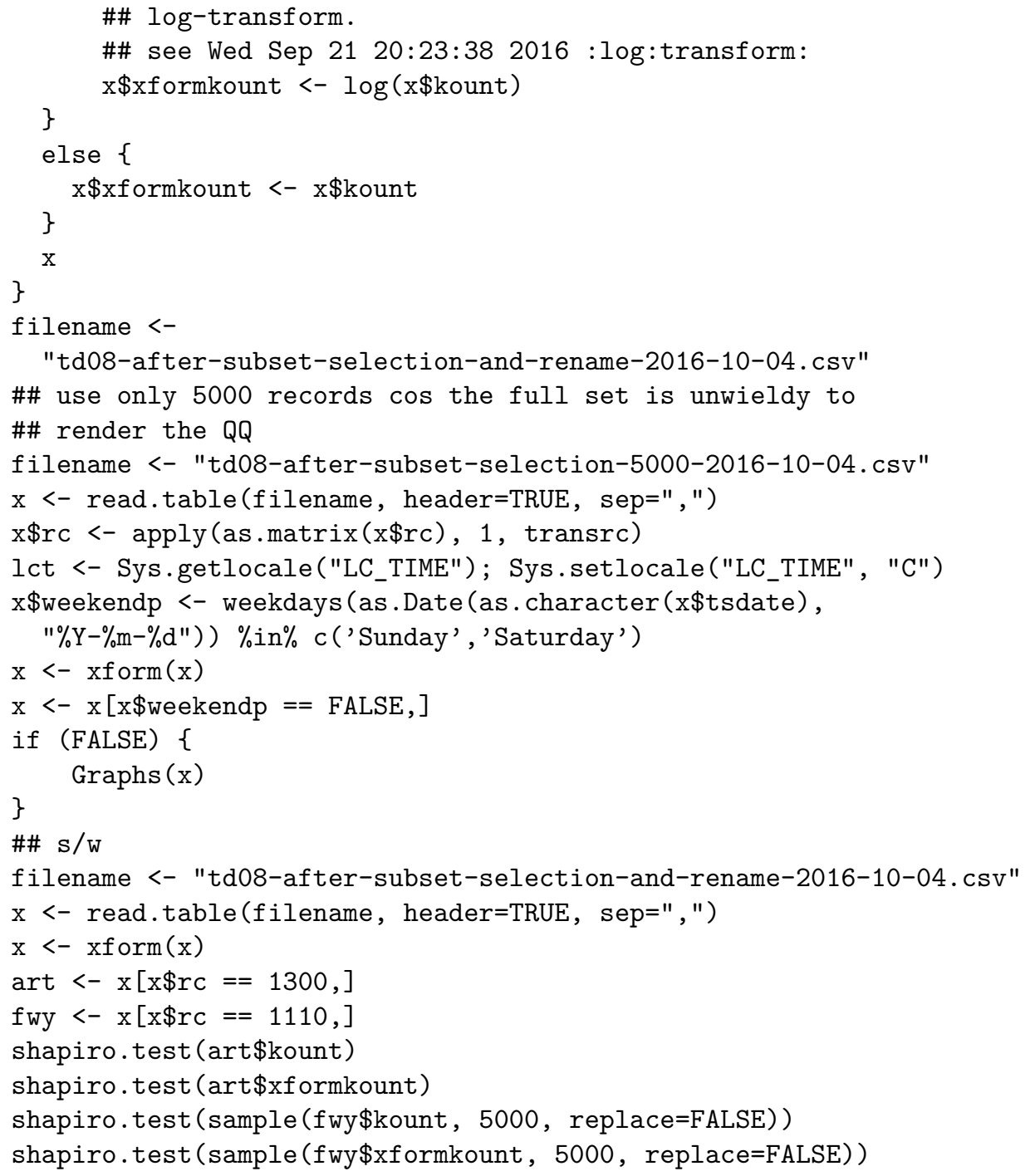

§A.6.5 Join the raw counts with our pseudo-LUR variables.

This task (pass 8 in the pipeline) is made easy by R's merge function. It's fast, too (1 minute). The input, lur-min.csv, is easily extracted from the GIS layer.

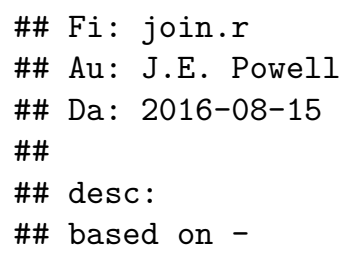




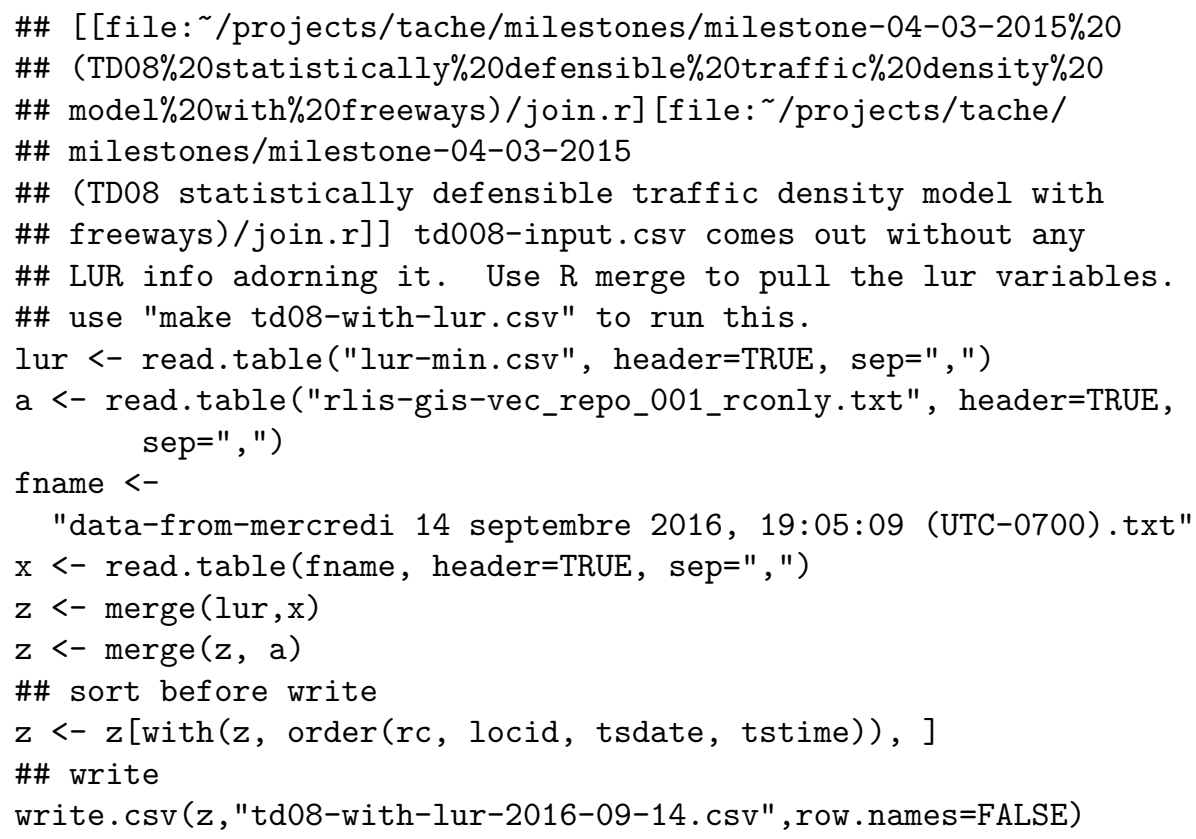

This computation takes a long time (about 8 hours). There is surely a better and fast way to do it in R, because using rbind is almost always a sign that you've broken the $\mathrm{R}$ paradigm.

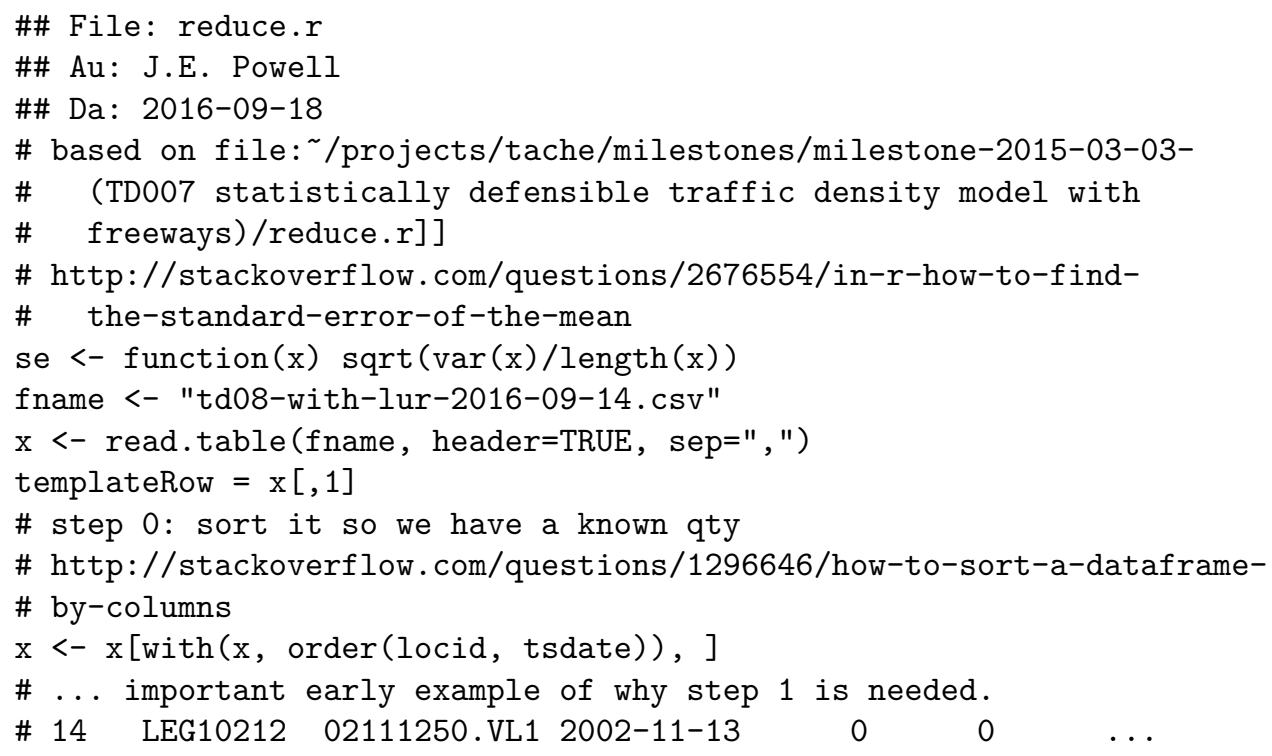




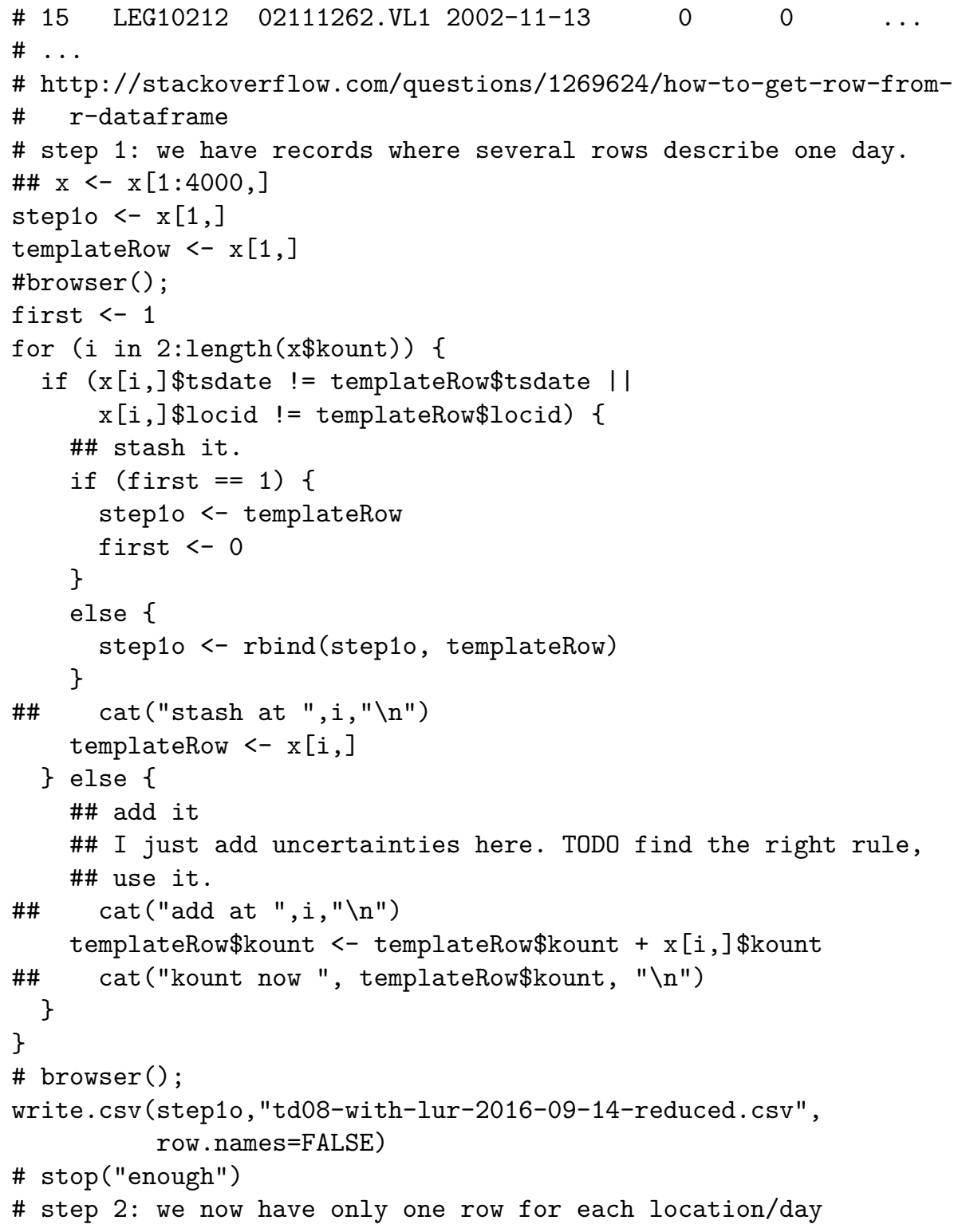

$\S$ A.6.7

Translate road class (RC) identifiers to name

\#\# File: transrc.r

\#\# Au: J.E. Powell

\#\# Da: 2016-09-27

\#\#

\#\# Based on src/roadclassdb.cc

\#\# (a) = "In the area maintained by the City of Portland only"

\#\# (b) = "Private right-of-way exists"

\#\# (c) = "With rapid transit"

\#\# (d) = "to local street contor (Portland

$\# \#(e)="-$ named, but without valid addressing (Clackamas County only, subarea = 'C')"

$\# \#(f)=$ "-unnamed and without valid addressing (Clackamas County only, subarea $={ }^{\prime} \mathrm{C}$ ')"

$\# \#(g)=$ "with valid address range and street name (in the area maintained by the City of Portland only)" 


\section{A.6. SOURCE CODE}

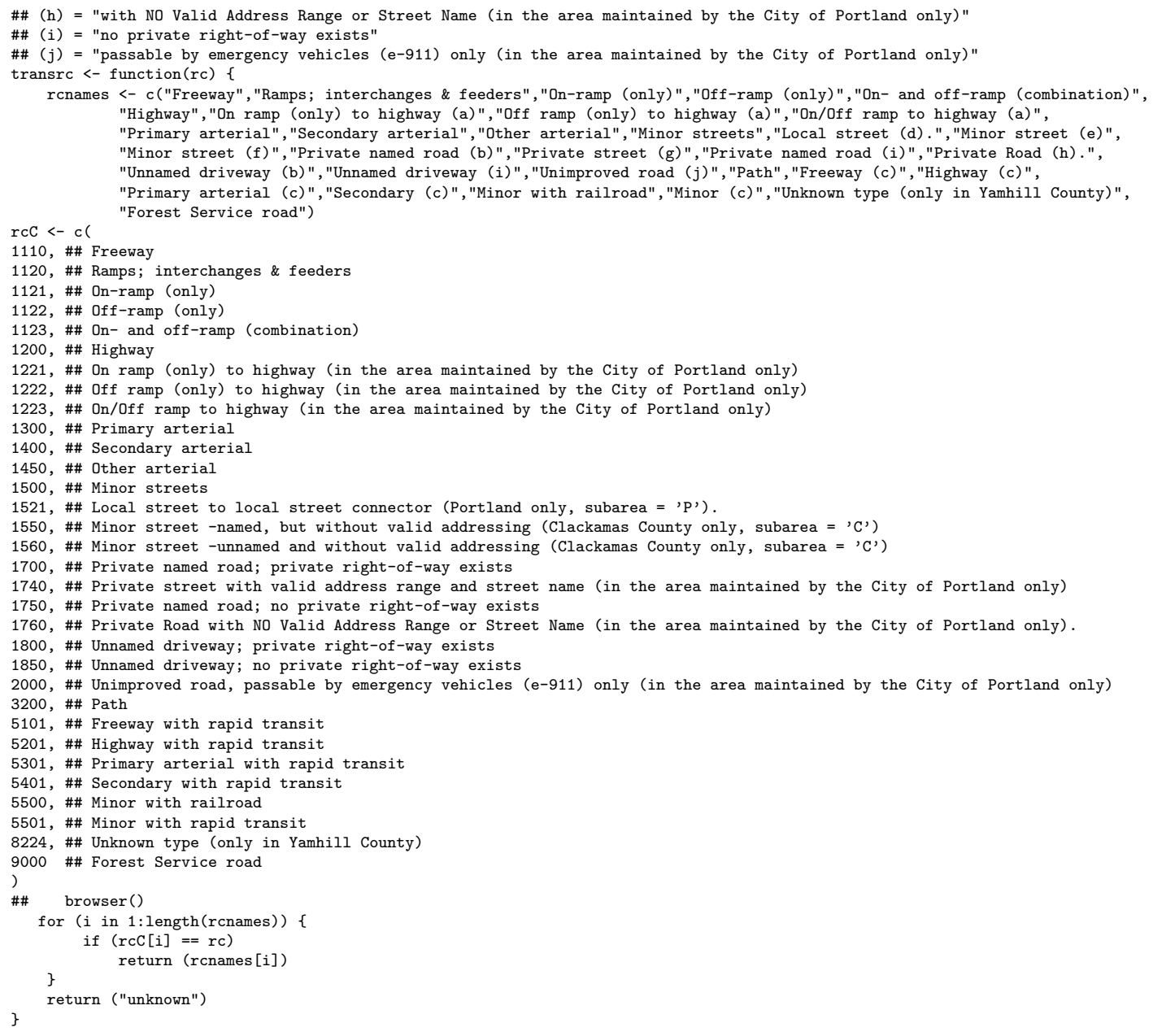

\section{$\S$ A.6.8}

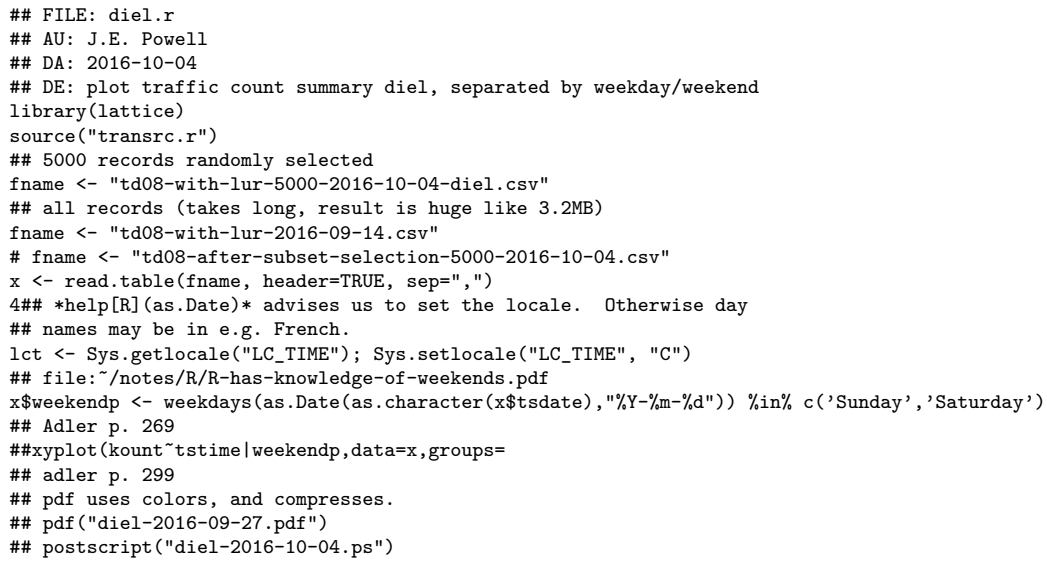


$\mathrm{x} \$ \mathrm{rc}<-$ apply (as.matrix $(\mathrm{x} \$ \mathrm{rc}), 1$, transrc)

png (filename $=$ "diel-2016-10-04.png" , width $=4096$, height $=4096)$

trellis . par. $\operatorname{set}$ ( fontsize=list (text=7))

\#\# xyplot (kount tstimelas. factor ( $r c)$, data $=x$, groups=weekendp, auto . key=TRUE, scales=" free")

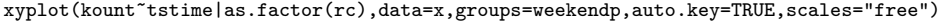

$\operatorname{dev} \cdot \operatorname{off}()$

Make a random subset of the raw data for validation

Note that we seed the Random Number Generator (RNG) to make our subset selection reproducible. We use $\mathrm{R}$ because it is known to have a superior RNG compared to some other systems.

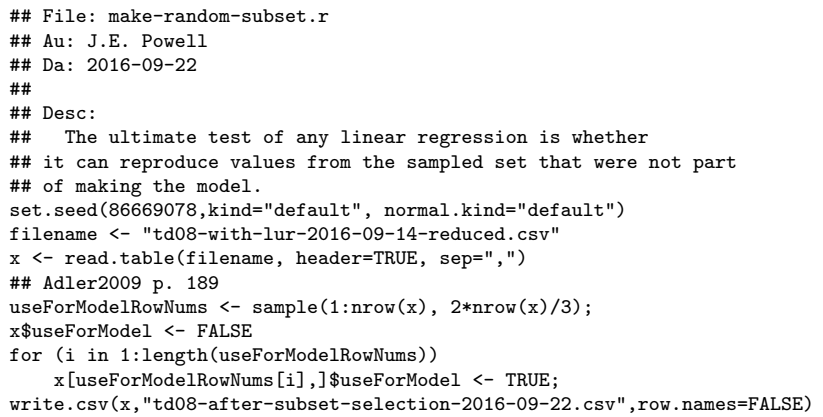

The above code makes a reduced set: counts aggregated to the day level. To mark a random subset of the hourly set, used in deriving and testing the canonical diel cycle, the following code was used.

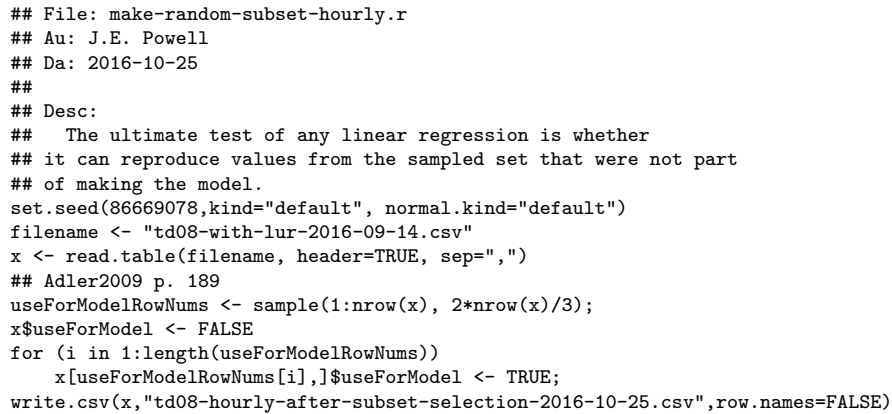

Warning: this later code takes time, about 24 hours on a fast research host. That time is all spent in this loop: 
$>$ for ( $i$ in $1:$ length (useForModelRowNums))

$+\quad \mathrm{x}[$ useForModelRowNums [i], ]\\$useForModel <- TRUE;

suggesting that $\mathrm{R}$ is not optimized for simple iteration over a lot $\left(1.1 \times 10^{6}\right)$ of rows.

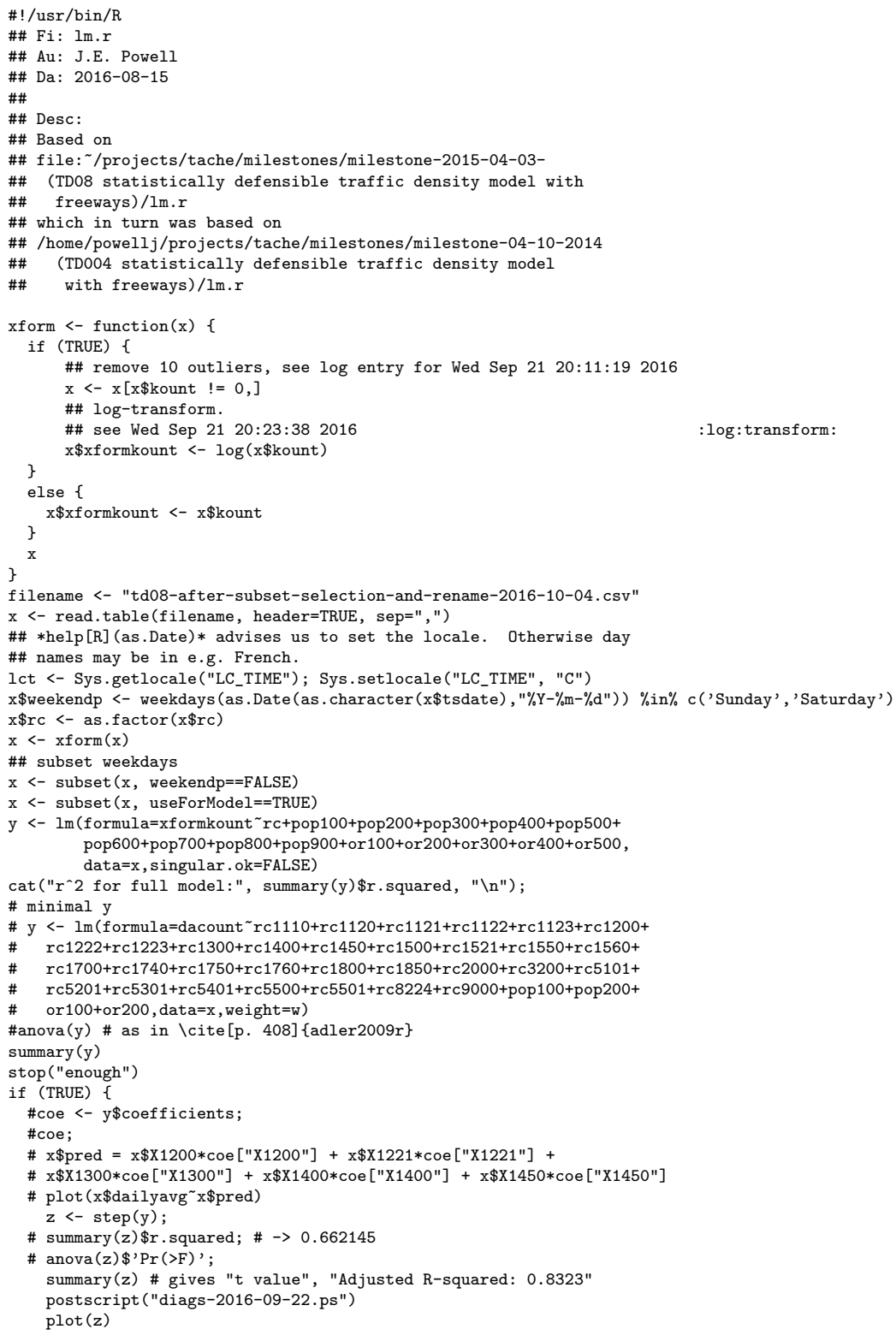


The values below $(594,264$, etc) were multiplied by 2 to obtain the values in Table 3.2 because we found that the average duration of one count (recorded under one countid) is about 48 hours in TDAT.

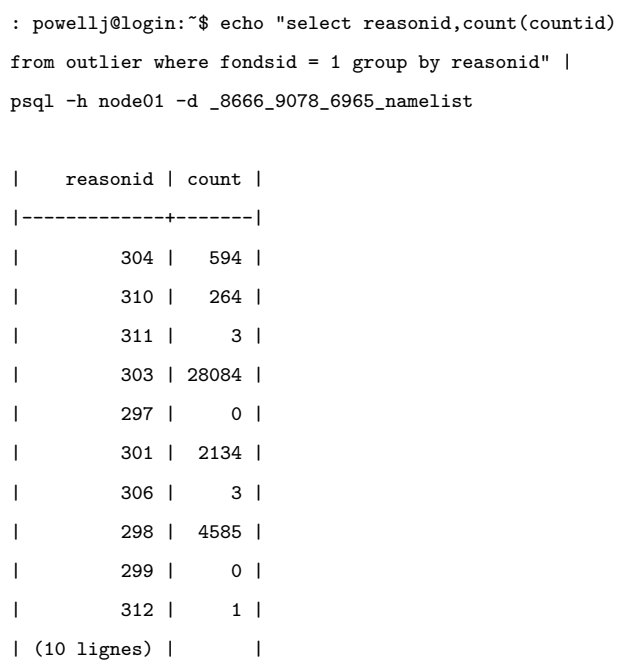

We call this pipeline "TD0N" - traffic density, $\mathrm{N}$ being some integer. We can be more specific, the version presented in this appendix is our eighth, so we call this one "TD08". 
Some interesting statistics.

- 80389 : number of potential count sites located in the study area (TDAT).

- 9352 : number of potential count sites where someone at PBOT allocated a countid (indicating an intention to count I presume) (TDAT).

- 7767 : number of actual counts sites, where a counter was actually laid on the road and read off into the database (TDAT).

- 48 : average number of hours that a counter was left on the road (TDAT).

- 15 : the usual bin size for the counters (in minutes) (TDAT).

- 60 : a bin size also used for some few counters (in minutes) (TDAT).

- 21 : approximate number of years of traffic count data contained in the PBOT database (starting in 1986, ending in 2006) (TDAT)

- 11072 : lines of $\mathrm{C}++$ code written to produce the maps and statistics reported here.

- 8 : minutes to read all counts in PORTAL-FHWA

- 12 : gigabytes of raw ASCII data in PORTAL-TOTAL

- 885 : megabytes of SQL in TDAT 
- 1128: CPUs Total in the Gaia cluster (localhost/Ganglia snapshot of 2014$11-26)$

- 94: Hosts (total) in the Gaia cluster (localhost/Ganglia snapshot of 2014-1126)

- 105178: road segments in the PDX street map

- 2005 to 2014: years accumulated in PORTAL-TOTAL 
Source code for the TD0N pipeline forms the remainder of this document.

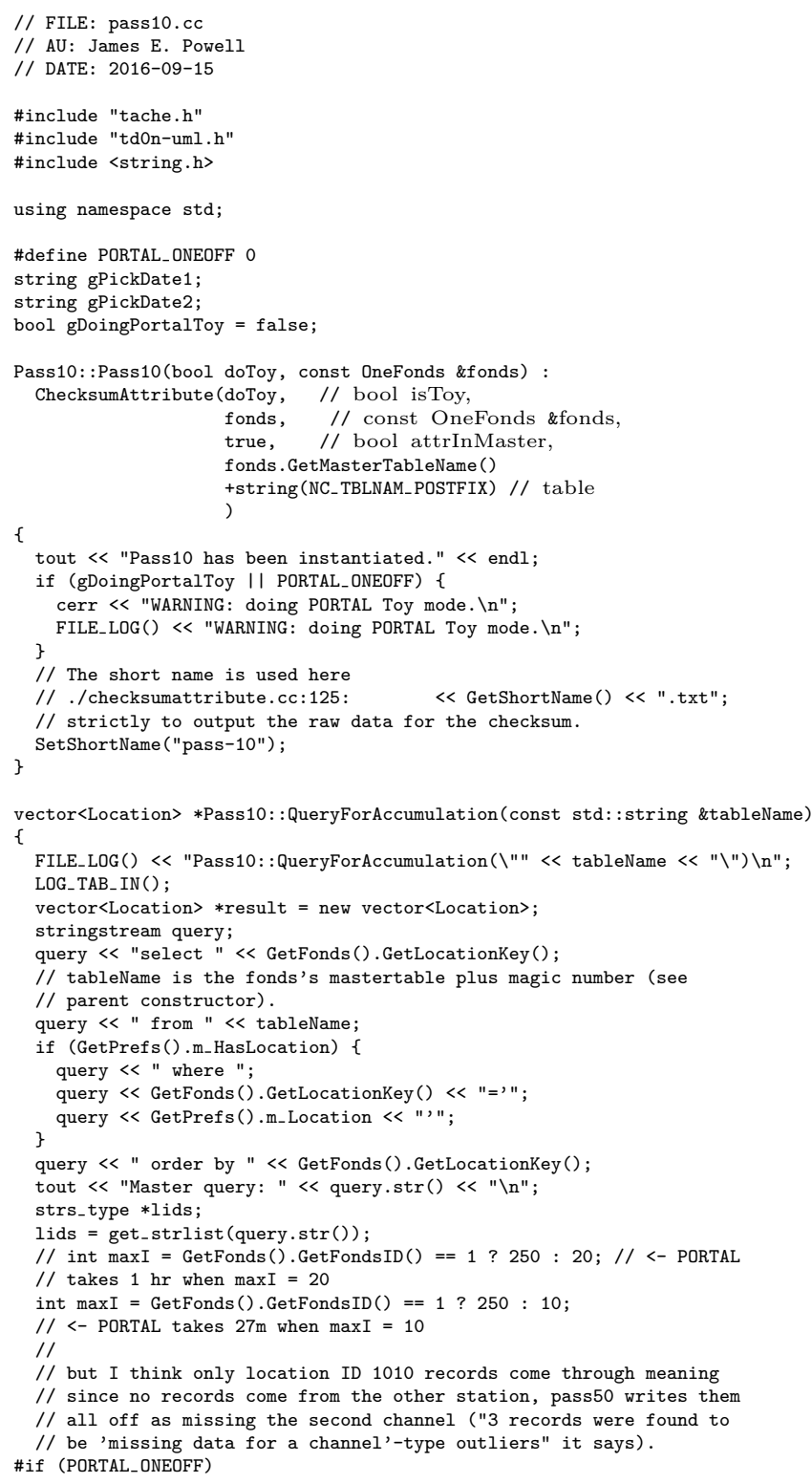




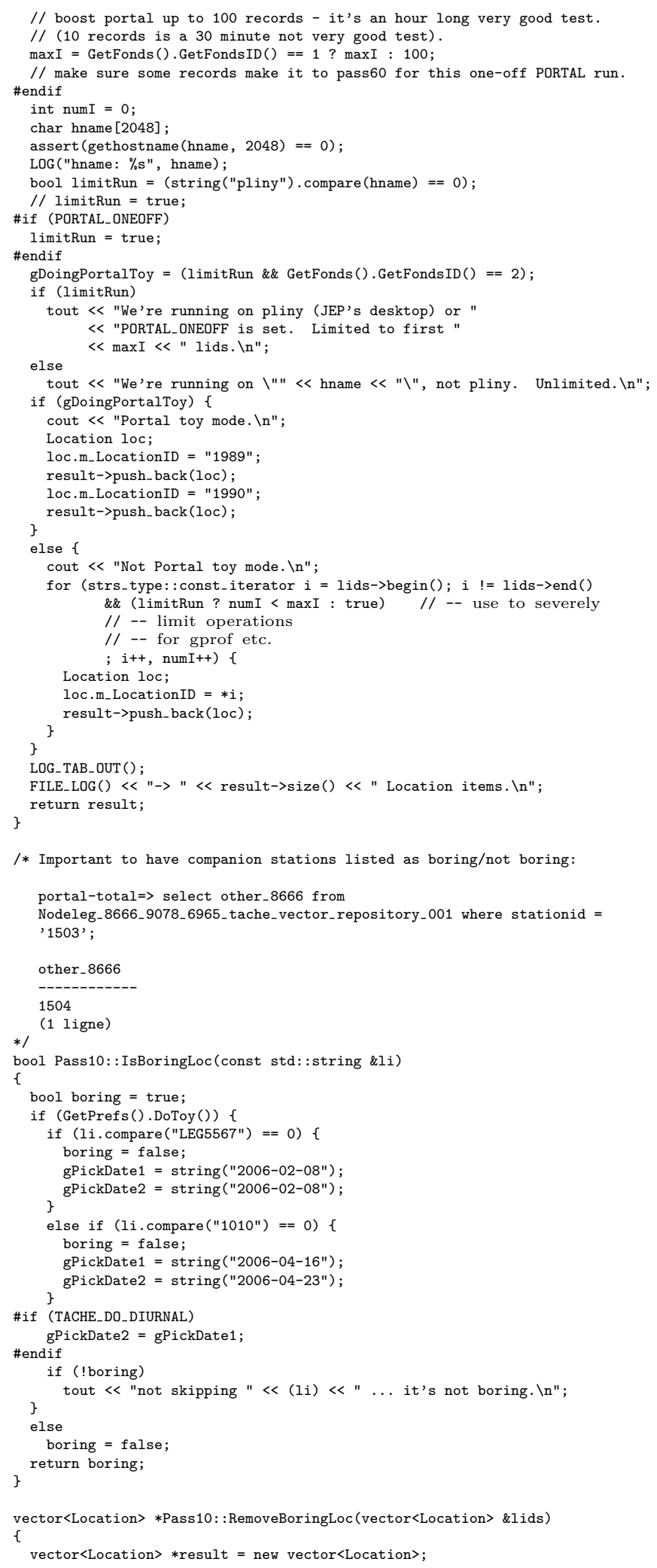




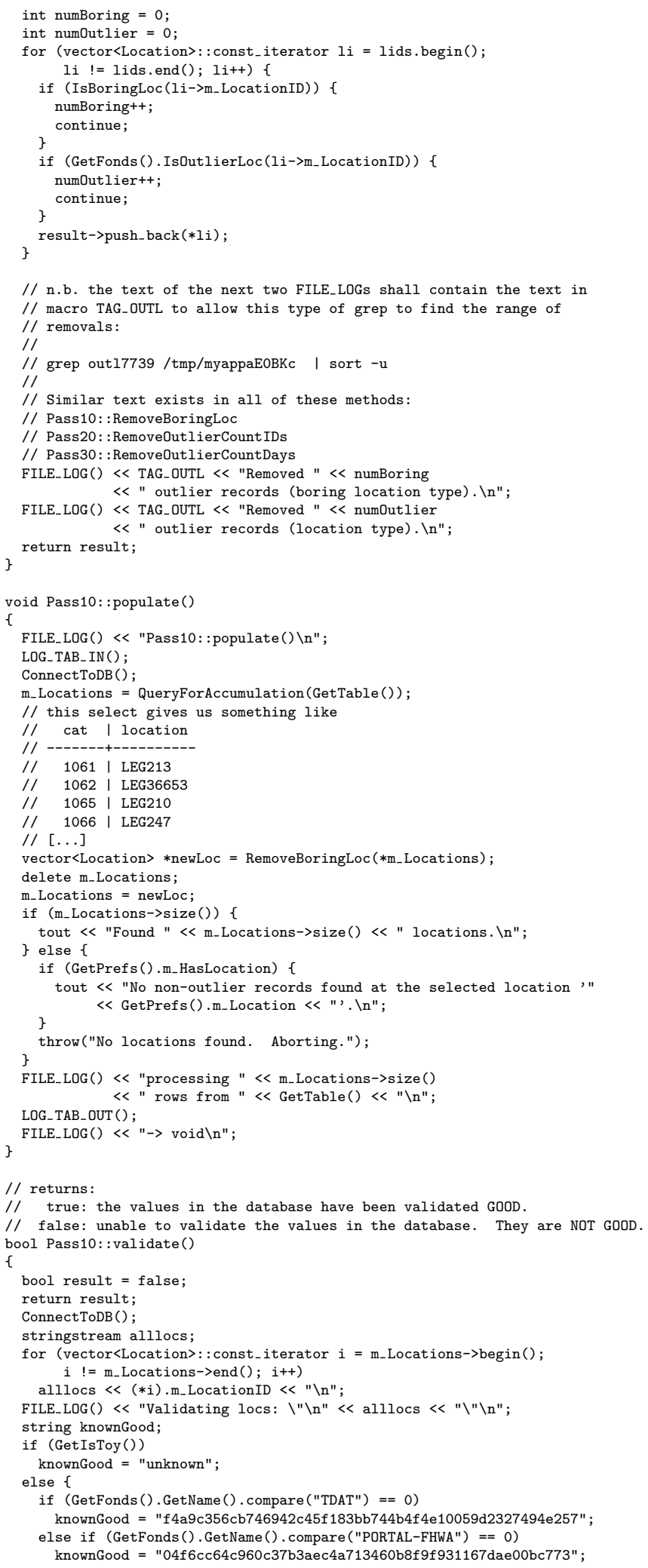




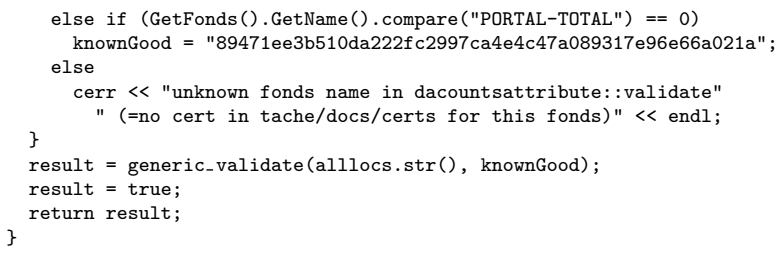




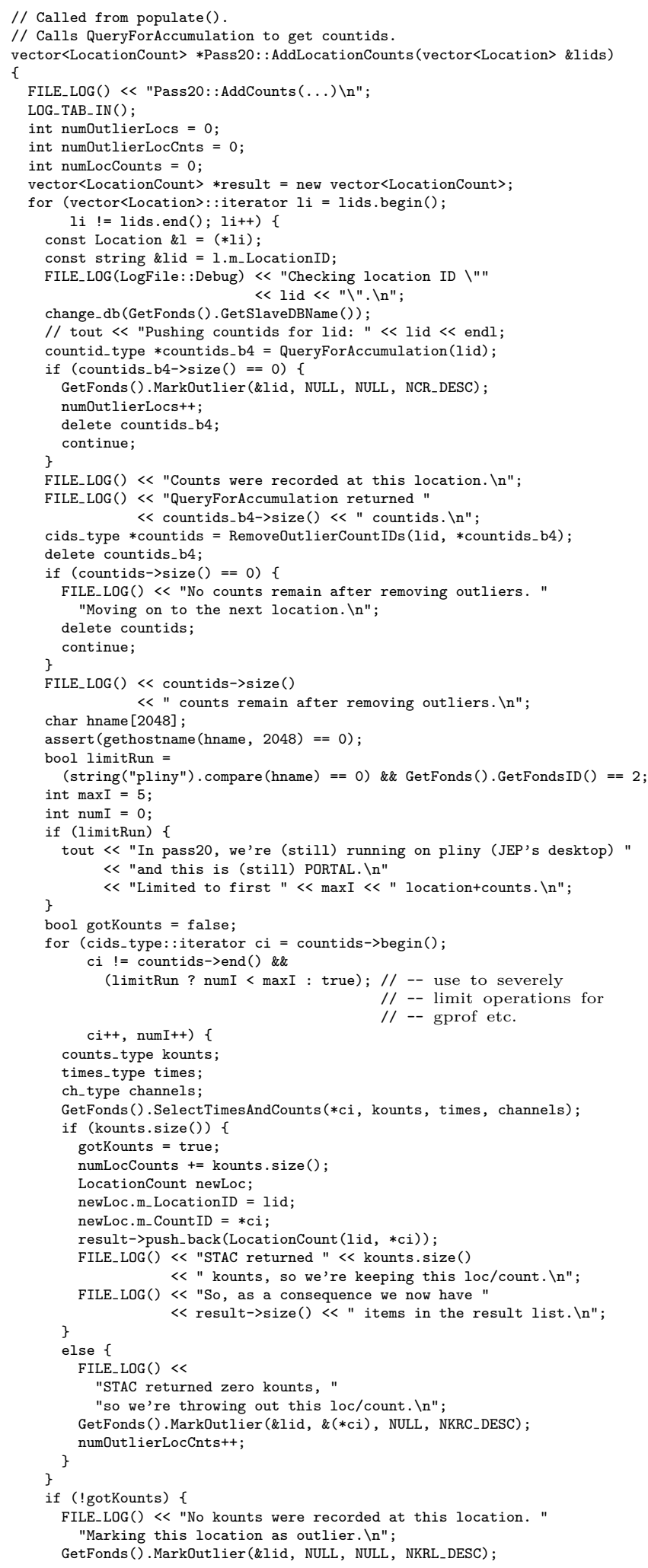




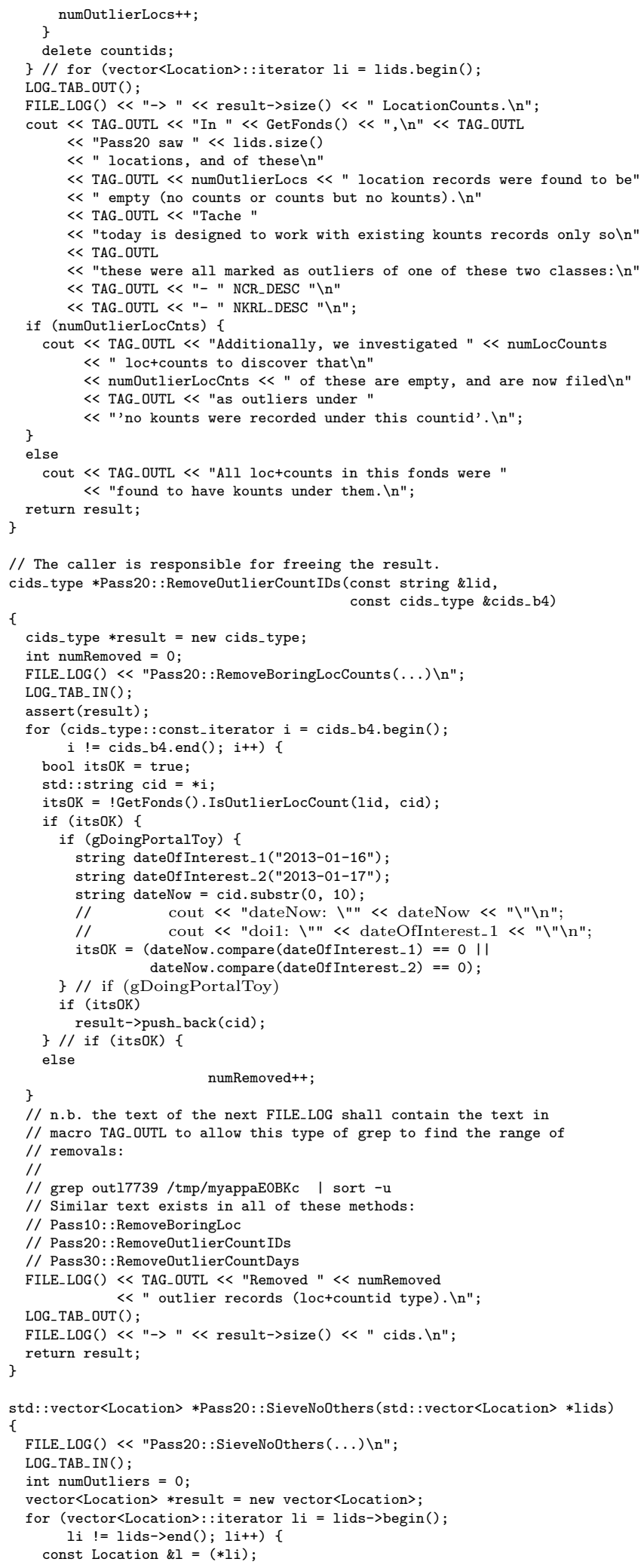




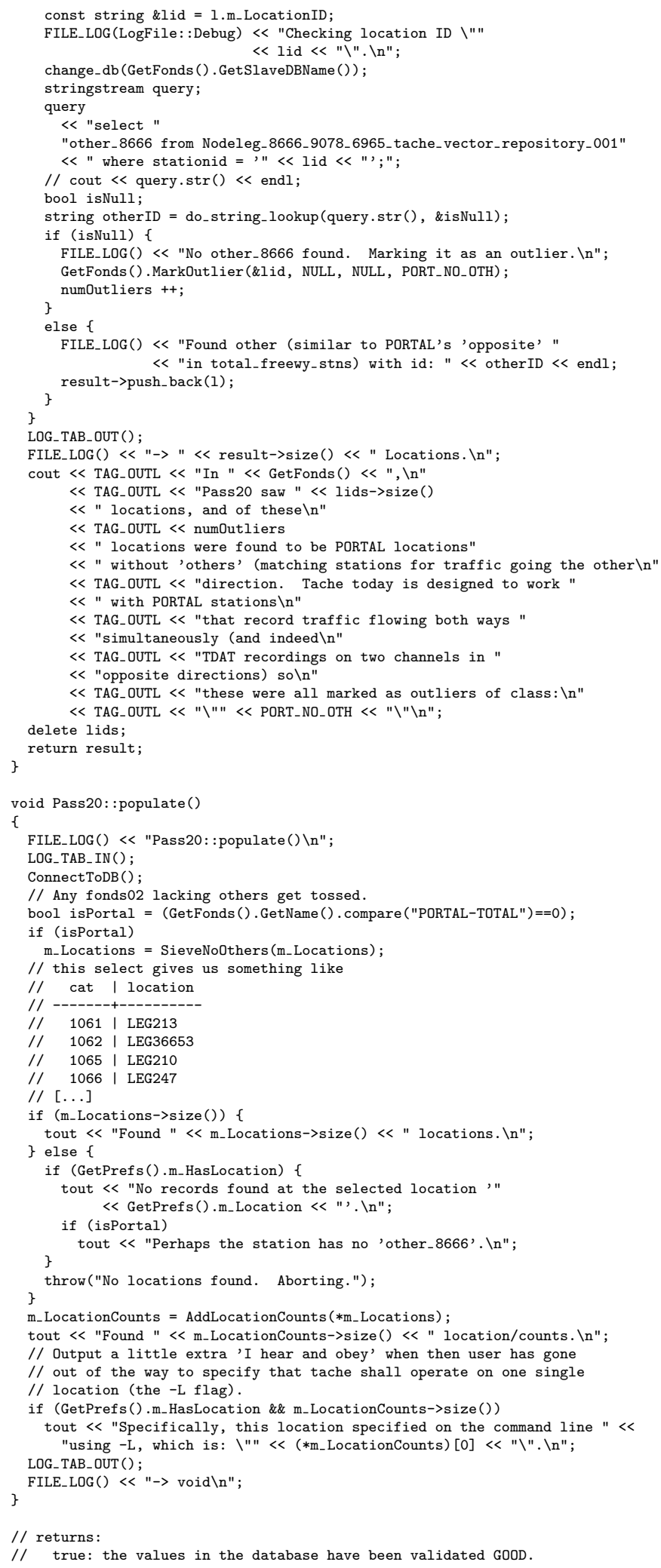




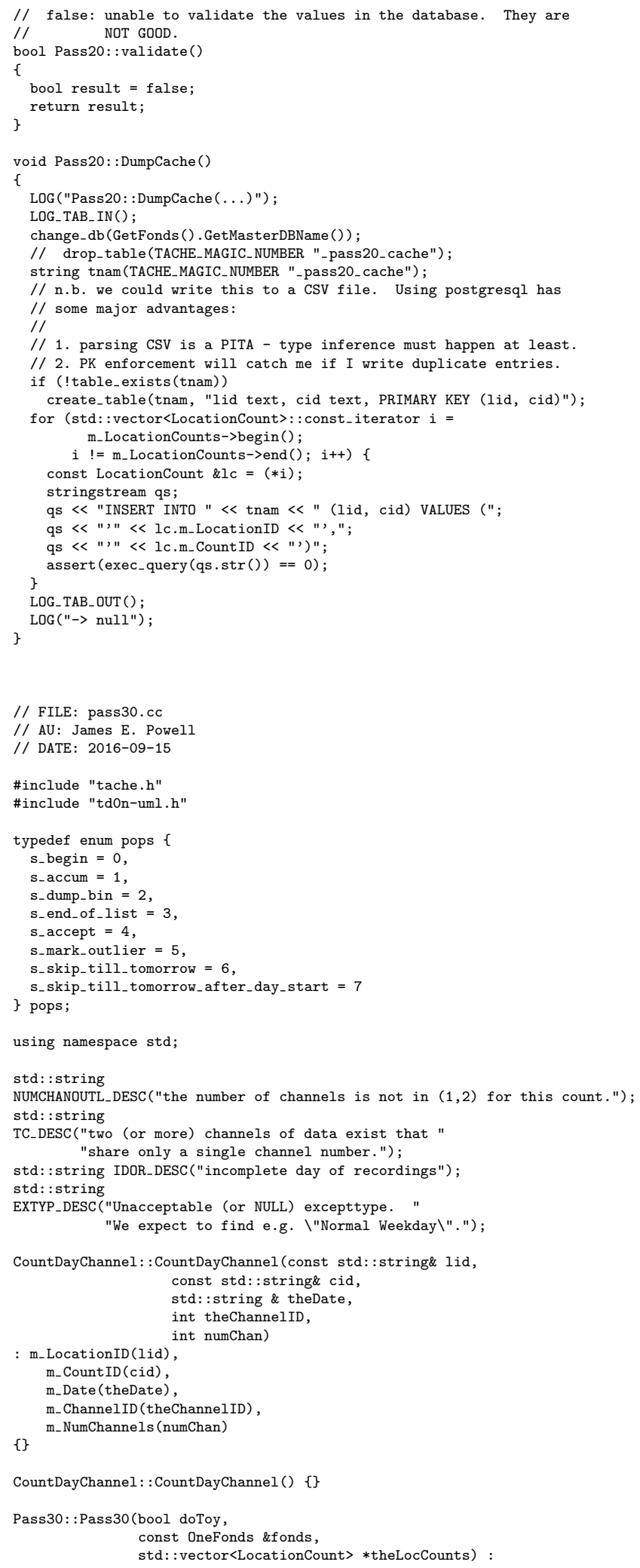




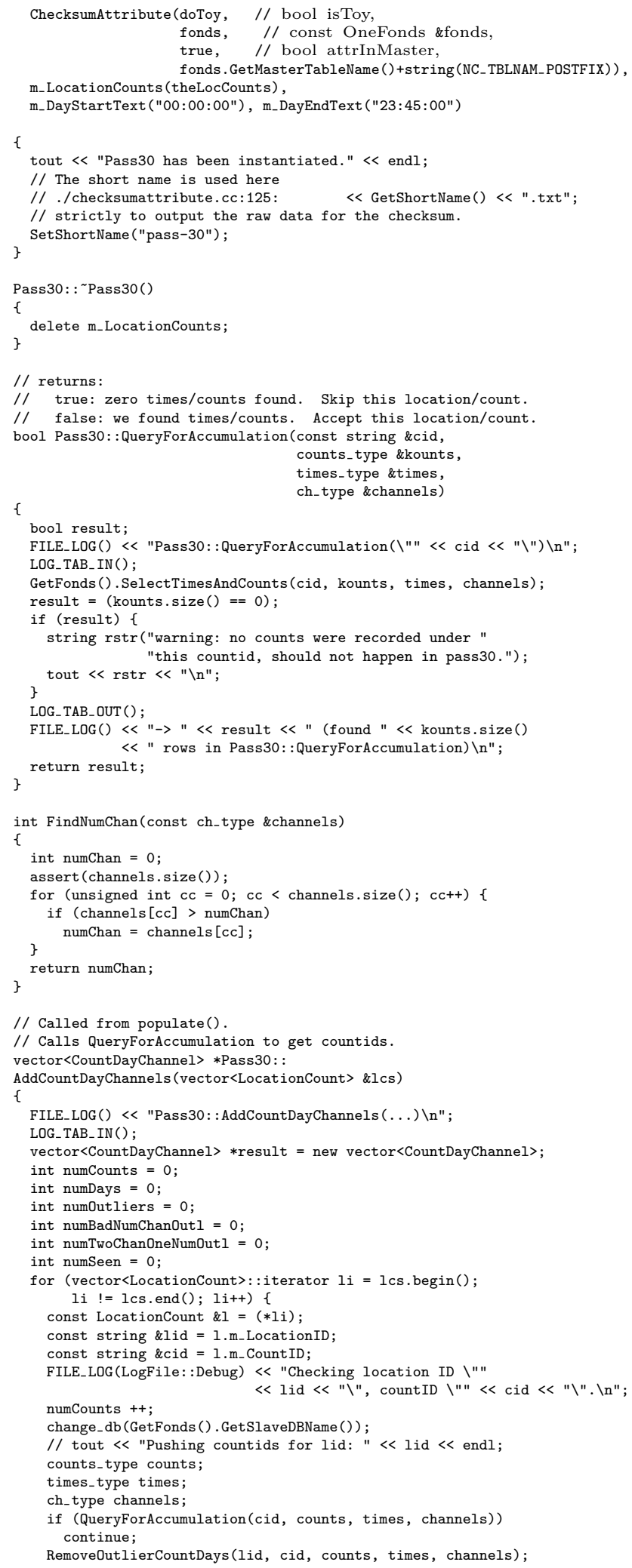


if (times.size ()$==0)\{$

FILE_LOG ()$\ll$ "All results were outlier. Moving to next location. $\backslash n$ "; \} continue;

FILE_LOG ()$\ll$ "Reading over " $<$ times.size ()$<<$

" records in the counts array resulting from the last SELECT. \n";

pops st $=$ s_begin $_{-}$

string fmt $=$ string $(" \% \mathrm{H}: \% \mathrm{M}: \% \mathrm{~S} ")$;

string dfmt $=$ string $(" \% \mathrm{Y}-\% \mathrm{~m}-\% \mathrm{~d} ")$

string dayOfKount;

int numChan = FindNumChan (channels);

// tache is designed for two cases: either a single channel with

// channel id 1, or two channels with channel ids 1,2 .

if (numChan $!=1$ \&\& numChan $!=2)\{$

// cerr $\ll$ "Warning: numChan $=" \ll$ numChan $\ll$ " in lid " $\ll$ lid

$/ / \quad<<"$ and cid " $<$ cid $<<" . \backslash n "$;

ILE_LOG ()$\ll<$ "Warning: numChan $="<<$ numChan $\ll<$ in lid $" \ll$ lid $\ll<$ and cid $"<<$ cid $\ll<" . \backslash n " ;$

GetFonds().MarkOutlier(\&lid, \&cid, NULL, NUMCHANOUTL_DESC) numBadNumChanOutl ++;

if (numChan < 0$)\{$

cerr $<<$ "Skipping negative channel count case. $\backslash \mathrm{n}$ ",

$/ /$ this never happens and if it did we would want to make an

// outlier record for it instead of blindly continuing.

\} assert (0);

string lastGoodTimestamp(" ")

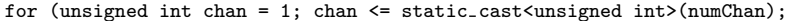
(chan++) \{

for (unsigned int $c c=0 ; c c<$ times.size ()$; c c++)\{$

assert (channels [cc] <= numChan);

if (channels $[c c] !=$ static cast $\langle$ int $\rangle($ chan $))\{$

\#define SPAMOK

\#if (SPAMOK)

// Spammy, but useful for debugging when -L flag is supplied.

FILE_LOG ()$<<$ "channels $["<<c c$

$$
\begin{aligned}
& \ll "]==" \ll \text { channels }[\mathrm{cc}] \\
& \ll " \text { which is != the current channel which is chan=" } \\
& \ll \text { chan } \ll \text { " so I'm skipping it. } \backslash \mathrm{n} " ;
\end{aligned}
$$

\#endif

\}

continue;

\#if (SPAMOK)

FILE_LOG ()$<<$ "channels $["<<c$ c

$\ll "]=="<<$ channels [cc]
$\ll<"$ which is == the current channel which is chan="

\#endif

< chan $\ll$ " so I'm NOT skipping it. \n";

string timesText $=$ MakeTimesText2(\&times $[\mathrm{cc}]$, fmt.c $\left.\mathrm{c}_{-} \operatorname{str}()\right)$;

string dayOfKount $=$ MakeTimesText2(\&times $[\mathrm{cc}]$, dfmt.c_str ()$)$

FILE_LOG ()$<<$ "at cursor=" $<<$ cc $\ll<$ st=" $<<$ st

$<<$ " timestamp: " $<$ day0fKount $<<" ~ "$

$<$ timesText $<<$ " lastGoodTimestamp="

$<$ lastGoodTimestamp $<<" \backslash \mathrm{n} " ;$

if $($ lastGoodTimestamp. compare (timesText) $==0$

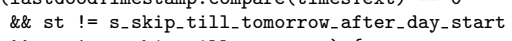

\&\& st $!=s_{-}$skip_till_tomorrow) \{

FILE_LOG()

$\ll<$ "We found two timestamps in a row, both with the same "

$<<$ "channel number. This is a case of "

$<$ "issue OFA: new outlier - two channels of records "

$<$ "that share a single channel number. $\backslash n$ ";

string dayOfKount $=$ MakeTimesText2(\&times [cc], dfmt.c.str ()$)$;

GetFonds().MarkOutlier(\&lid, \&cid, \&dayOfKount, TC_DESC, true); numTwoChanOneNumOutl ++;

\}

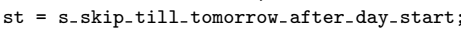

else

lastGoodTimestamp $=$ timesText;

bool doAgain = true;

while (doAgain) \{

doAgain = false;

switch (st) \{

case s_begin

// find a day start.

numDays ++;

numSeen $=0$;

if (m_DayStartText. compare (timesText) $==0)\{$

st $=s_{-}$accum

\}

doAgain = true 


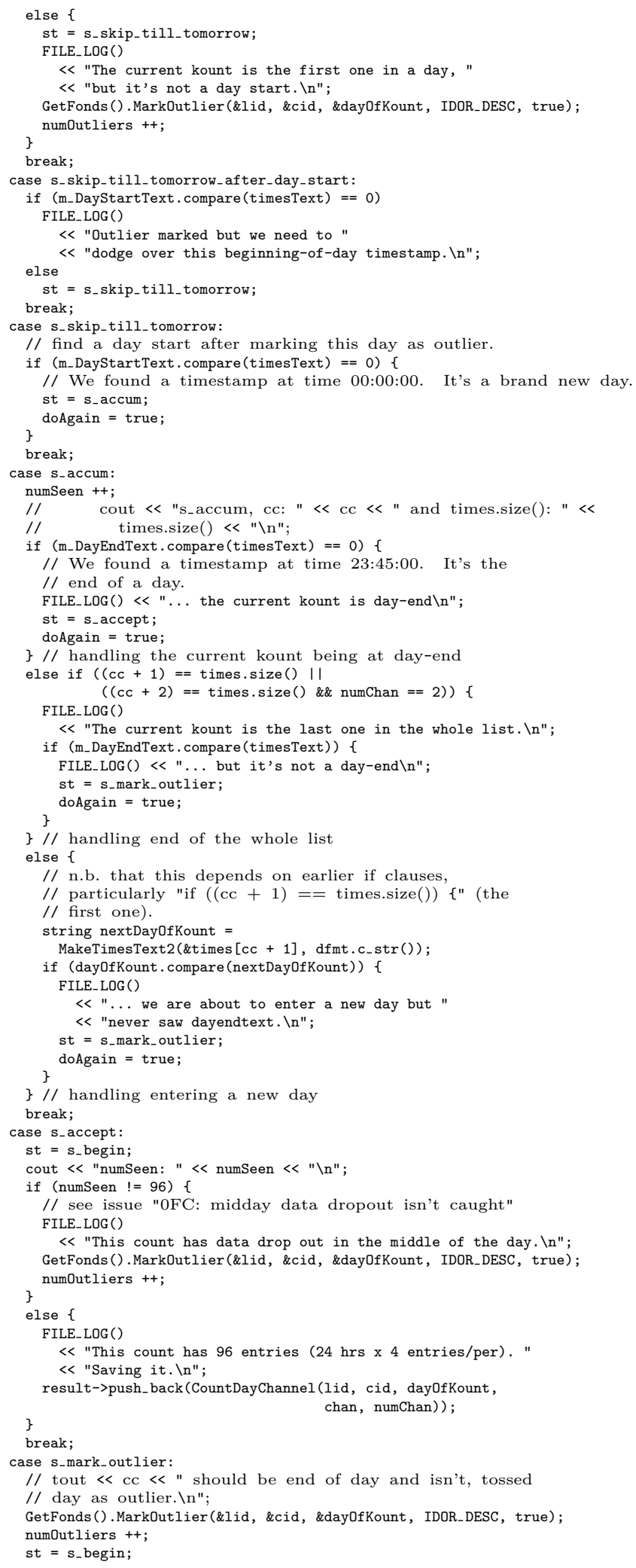




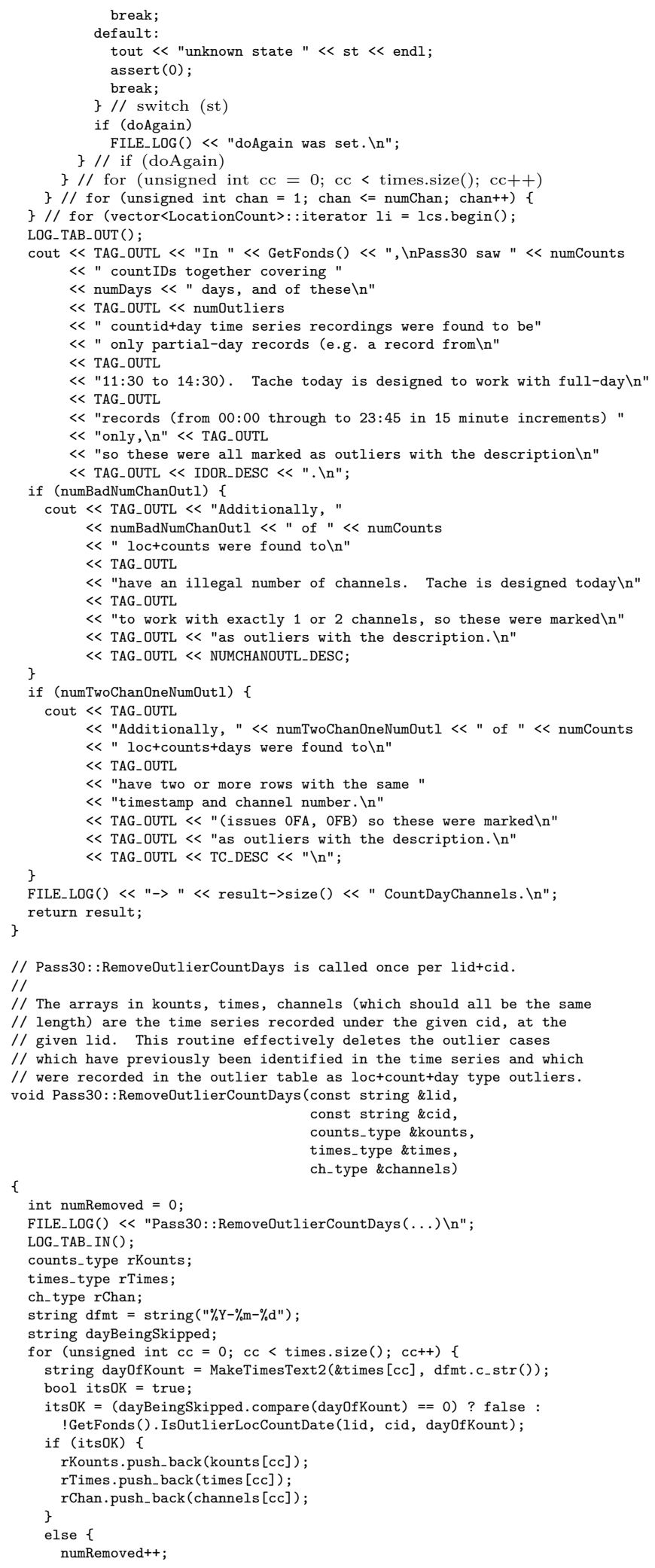




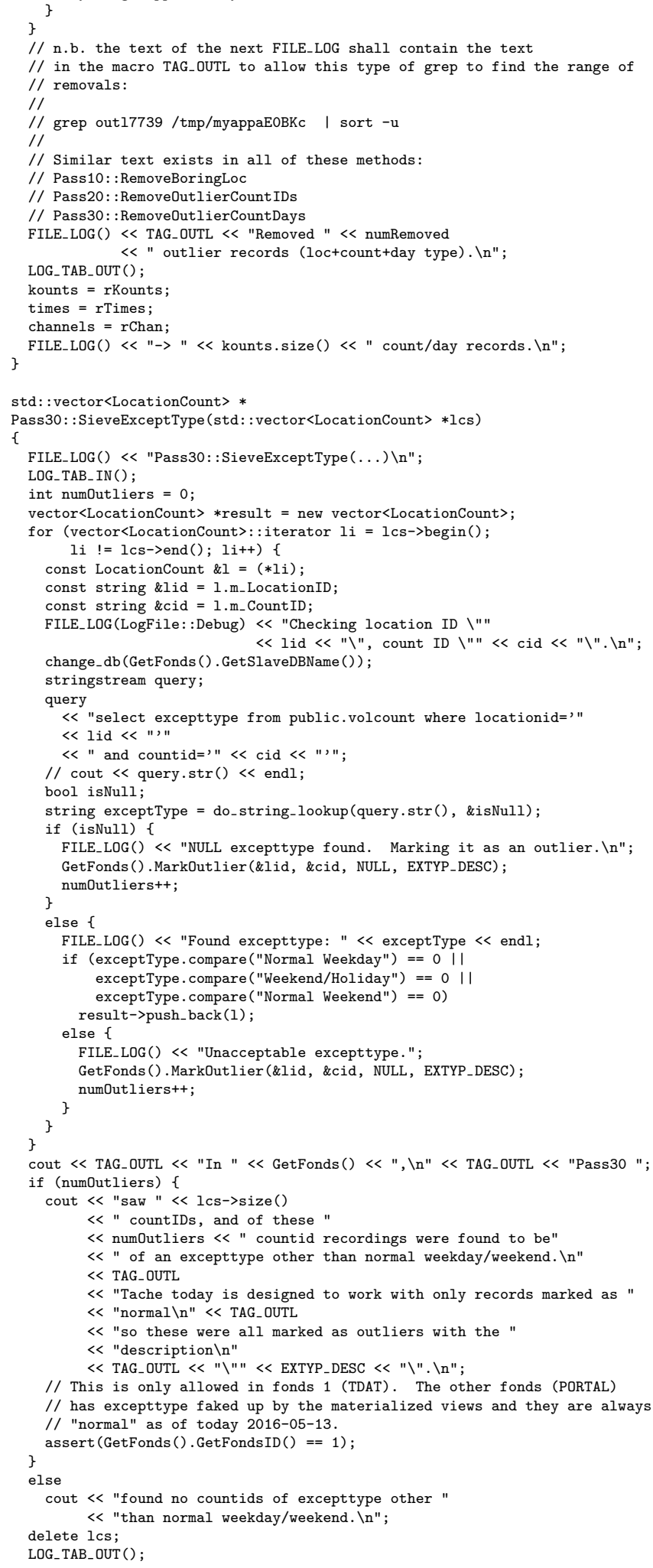




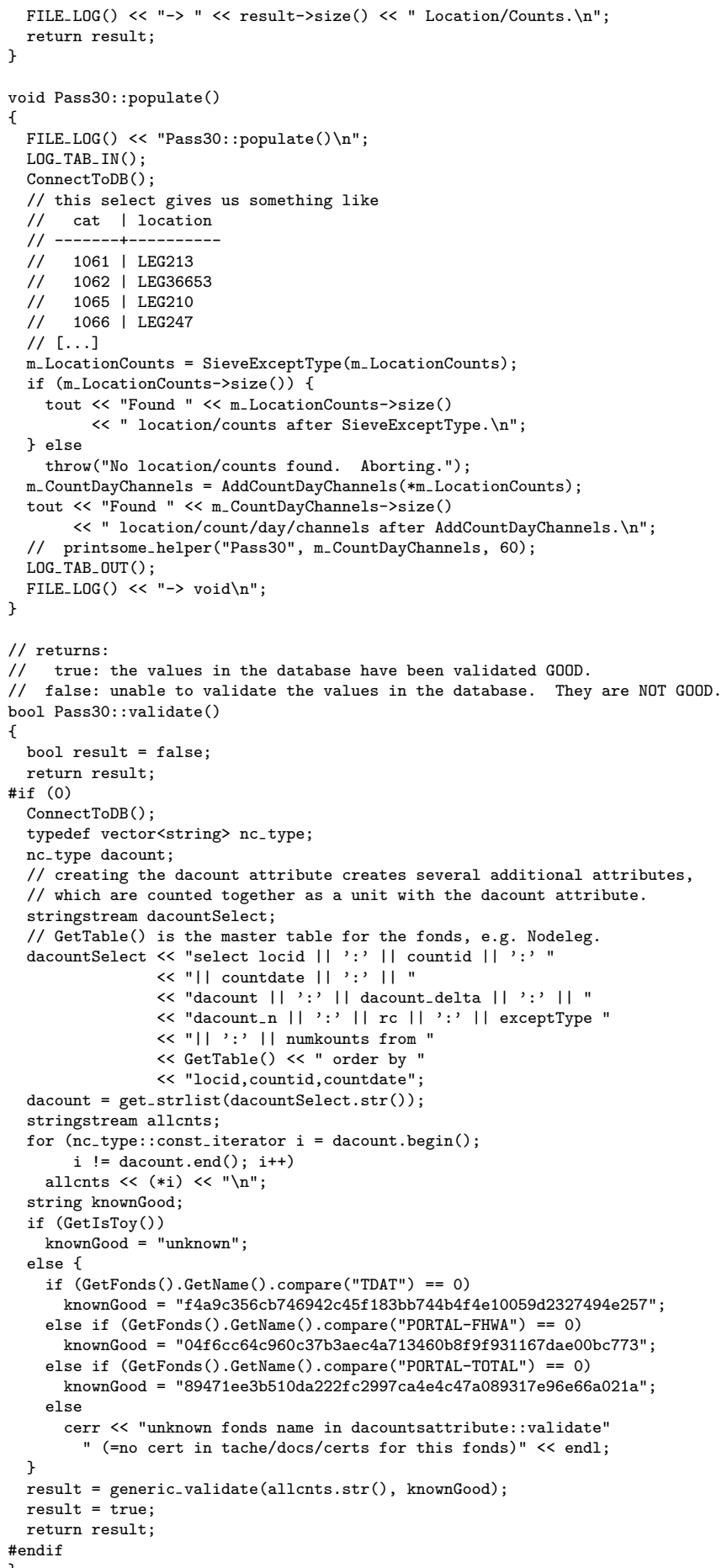




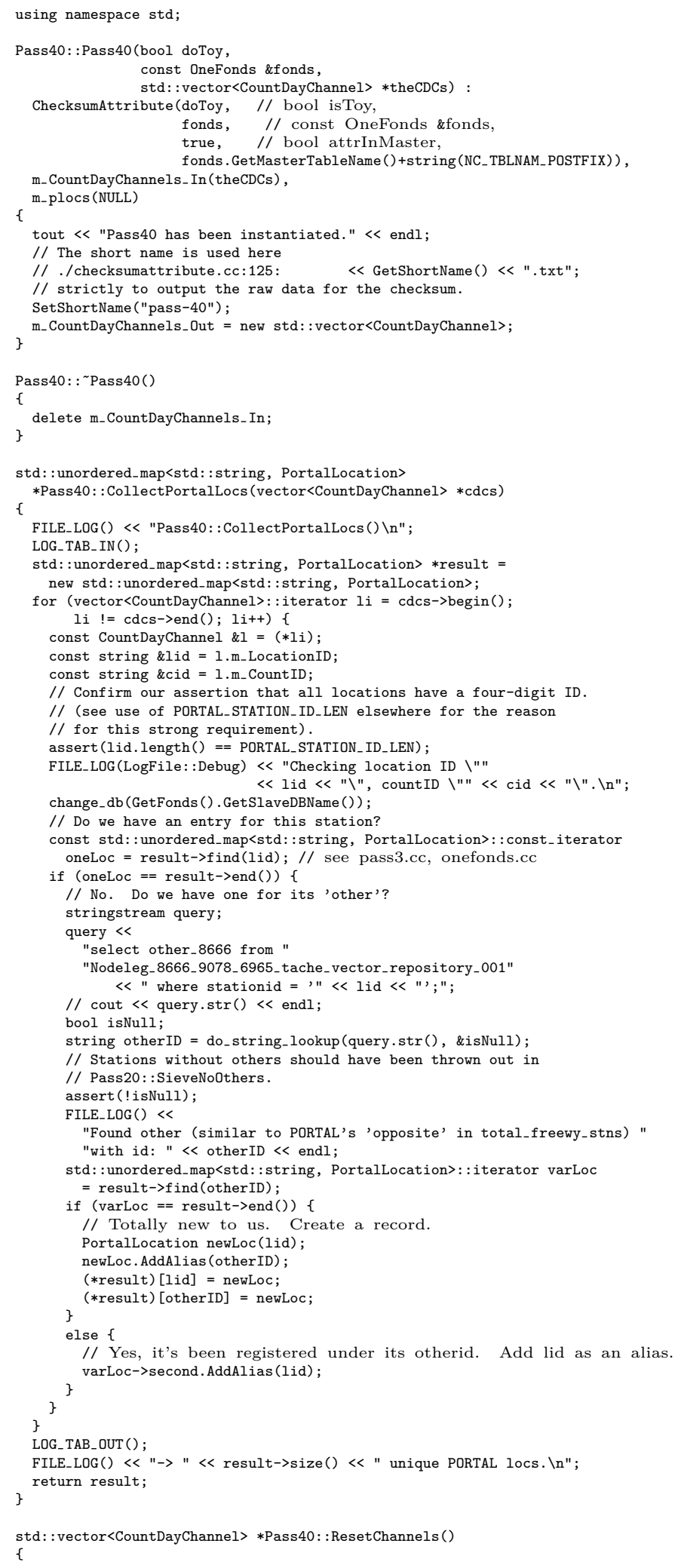



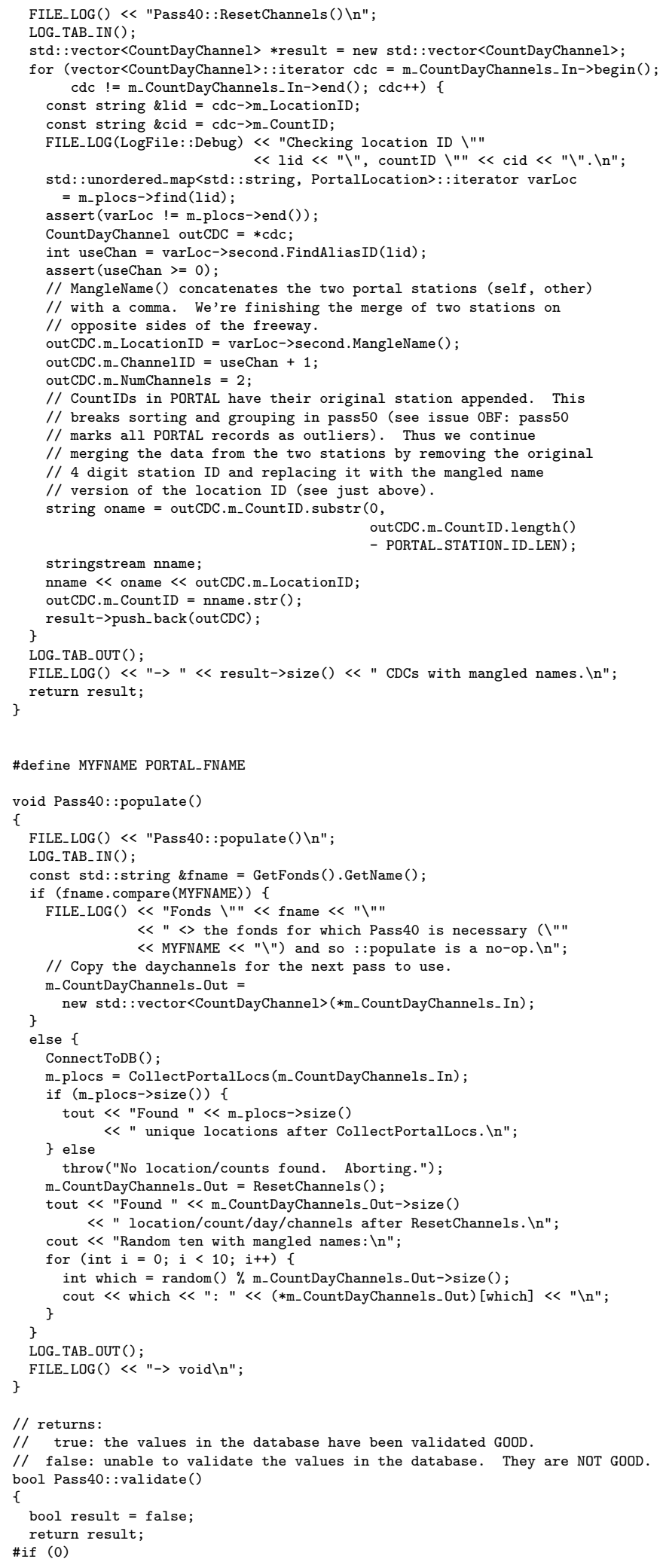


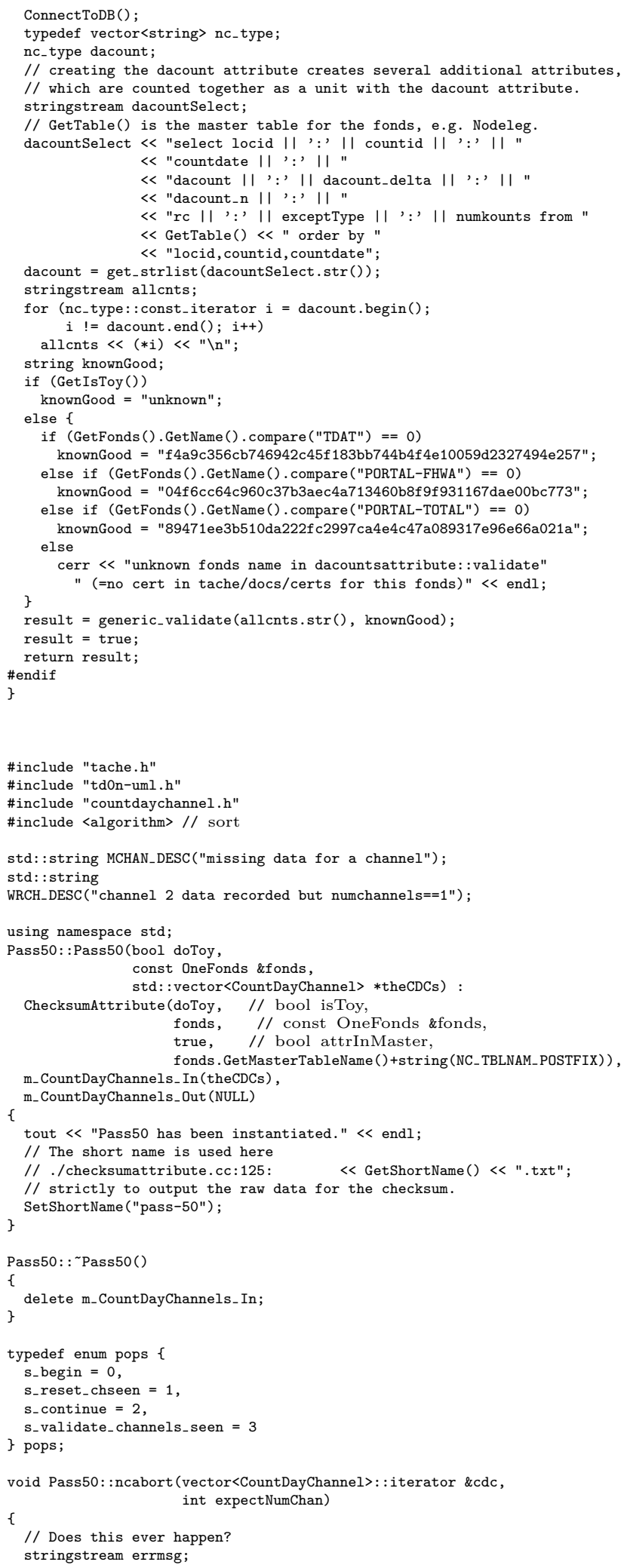




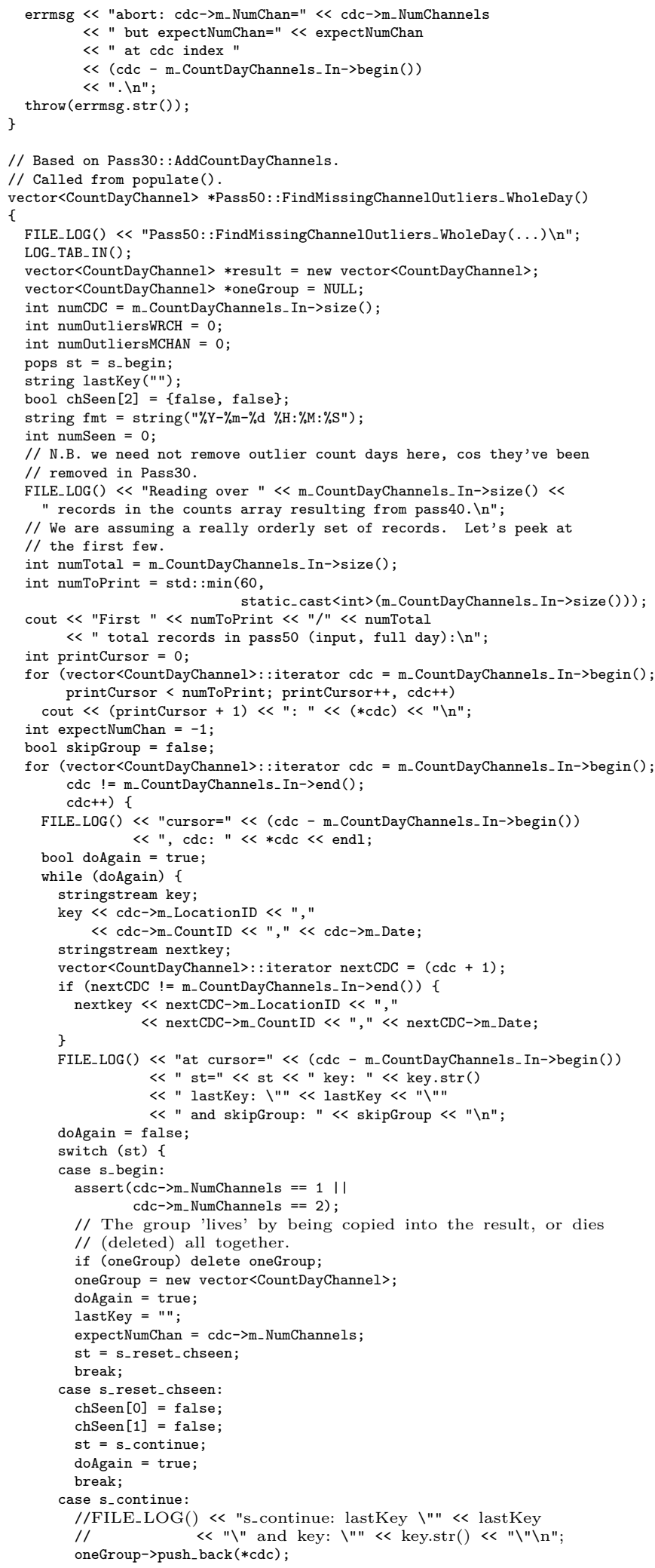


// We're stepping through a group of entries that all belong

$/ /$ to one count + day. Make sure these entries have the

// designated number of channels, picked by the first entry

$/ /$ in the group.

assert (cdc->m_ChannelID $==1|| c d c->m_{-}$ChannelID $\left.==2\right)$;

if (cdc->m_NumChannels $!=$ expectNumChan)

ncabort(cdc, expectNumChan);
$/ /$ This next assert may become an outlier, indicating duplicate

// records for one loc+cid+time+chan.

assert (chSeen [cdc->m_ChannelID - 1] == false);

FILE_LOG () $\ll$ "Recording presence of data in channel "

$<<$ cdc->m_ ChannelID $<<" \backslash \mathrm{n} "$

chSeen [cdc- $>\mathrm{m}_{-}$ChannelID - 1] = true;

if (cdc->m_NumChannels $==1 \mathrm{\&} \&$

cdc->m_ChannelID $!=1)\{$
GetFonds ().MarkOutlier(\&cdc->m_LocationID, \&cdc->m_CountID,

\&cdc->m_Date, WRCH_DESC, true)

numOutliersWRCH++;

skipGroup = true;

cerr $\ll$ "WARNING: The skipGroup = true code path has never "

$<<$ "been followed, \n"

$<<$ "because this type of outlier has not been seen as of $\backslash n$ "

\}

$\ll$ "2016-07-20. Proceed with caution. $\backslash \mathrm{n} "$

if $\left(\right.$ nextCDC $==m_{-}$CountDayChannels_In->end ()$)\{$

// End of the input set. Did we see all of the channels?

st $=\mathbf{s}_{-}$validate $e_{-}$channels $s_{-}$seen;

\}

doAgain = true

else if (nextkey.str (). compare(key.str ()$)==0$

II nextkey. $\operatorname{str}()$. length ()$==0$ )

FILE LOG ()$<$ "still in group... In" ;

else \{

FILE_LOG() $<$ "End of a group. Did we see all of the channels? \n";

st $=$ s $_{-}$validate channels $_{-}$seen;

\}

doAgain = true

break;

case s_validate_channels_seen:

numSeen $=0$;

if (skipGroup) \{

FILE_LOG() $<$ "Done skipping a group. $\backslash n$ "

st $=$ s_begin; $_{\text {- }}$

skipGroup $=$ false; continue;

for (int $i=0 ; i<c d c->m_{-}$NumChannels; $i++$ )

if (chSeen [i])

numSeen++;

FILE_LOG ()$\ll<$ "st=s_validate shannels $_{-}$seen, and numSeen is $" \ll$ numSeen $<<"$ and numchan=" $<<$ cdc->m_NumChannels $<<" \backslash n "$

if (numSeen $!=$ cdc- $>m_{-}$NumChannels) \{

GetFonds (). MarkOutlier (\&cdc->m_LocationID,

\&cdc $->m_{-}$Count ID

\&cdc->m_Date, MCHAN_DESC, true);

numOutliersMCHAN++

else \{

FILE_LOG() 《 "It's a good result. Saving this group. $\backslash n "$;

\}

copy (oneGroup->begin(), oneGroup->end (), back_inserter(*result));

st $=\mathbf{s}_{-}$begin;

break;

\} // switch (st)

if (doAgain)

FILE_LOG ()$\ll$ "doAgain was set. $\backslash n$ "

else

lastKey $=$ key.str () ;

3 // while doAgain

\} // for (vector<CountDayChannel $>$ : : iterator $c d c=m_{-}$CountDayChannels_ In $->\mathrm{b} \ldots$

if (oneGroup) delete oneGroup;

oneGroup = NULL;

LOG_TAB_OUT ();

FILE_LOG ()$\ll<"->"<<$ result->size ()$<<$ " CountDayChannels. In";

cout $\ll$ TAG_OUTL $\ll$ "In " $<$ GetFonds ()$<$ ", InPass50 saw " $<$ numCDC

$<<"$ count/day/channels, and of these $\backslash n "$

$\ll$ TAG_OUTL $\ll$ numOutliersMCHAN $\ll$ " records were found to be"

$\ll ", "$ " $<$ MCHAN_DESC $<<"$ '-type outliers, and " < numOutliersWRCH

$\ll<" \backslash n " \ll$ TAG_OUTL $\ll$ "were found to be,$" \ll$ WRCH_DESC

$<<"$ "-type outliers. \n";

eturn result;

void Pass50: : populate()

vo 


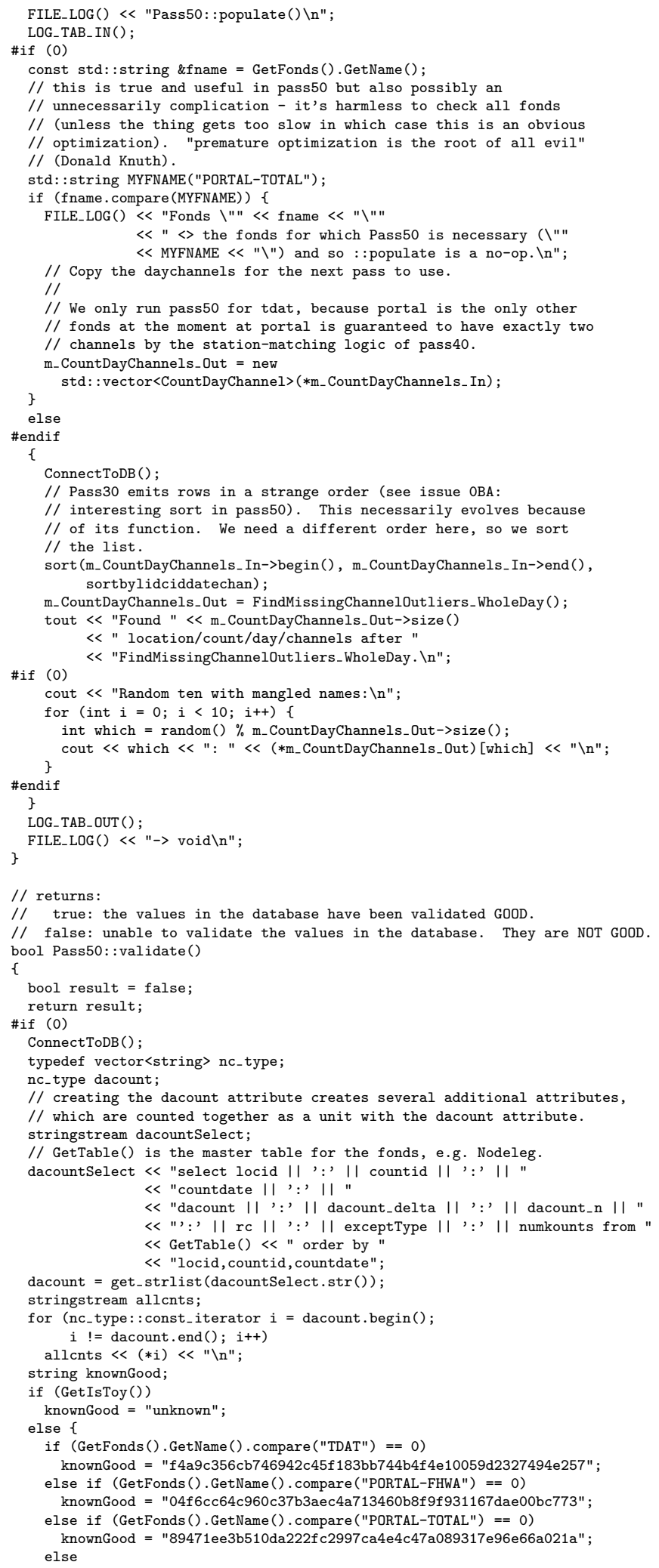




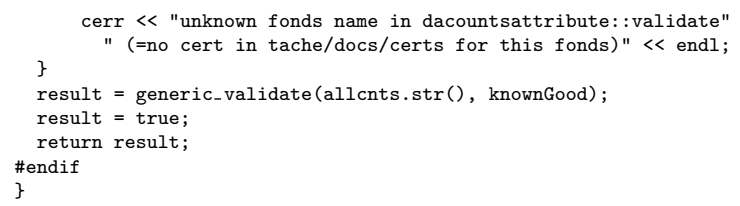




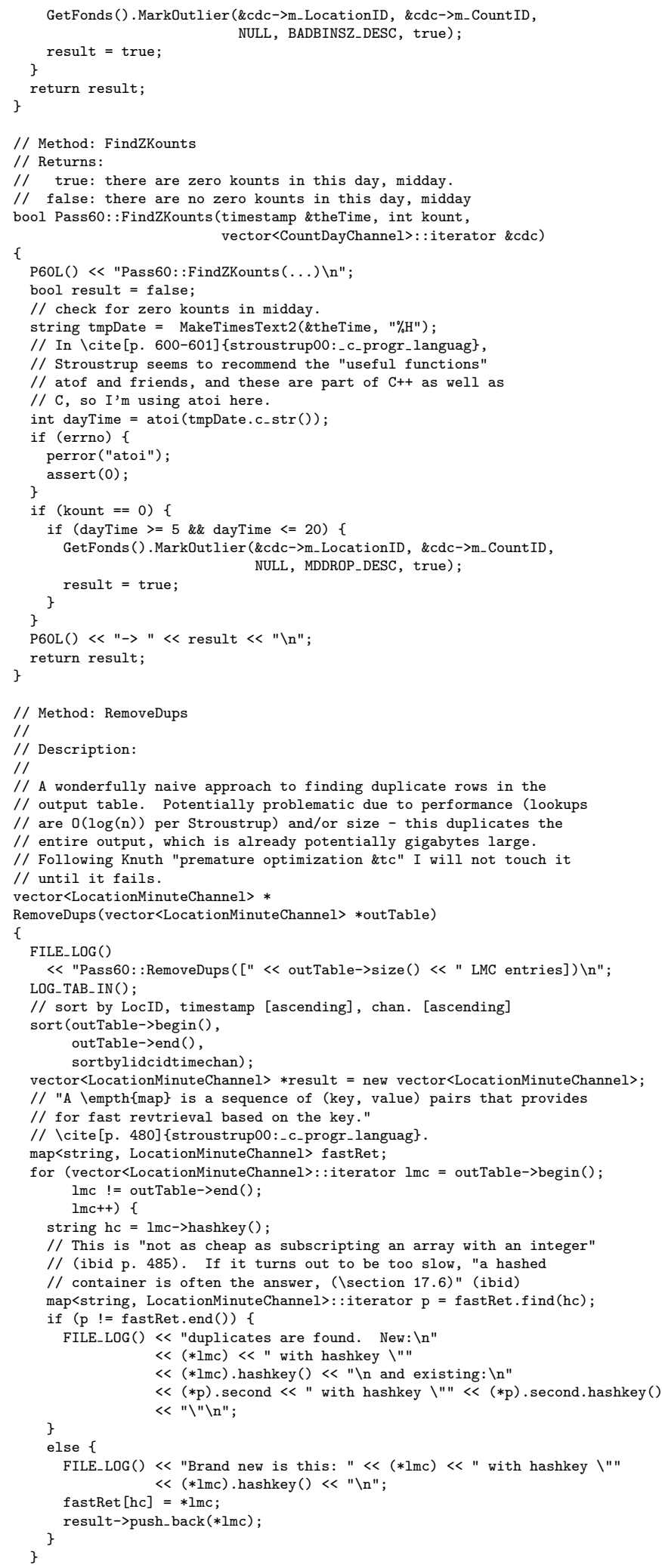




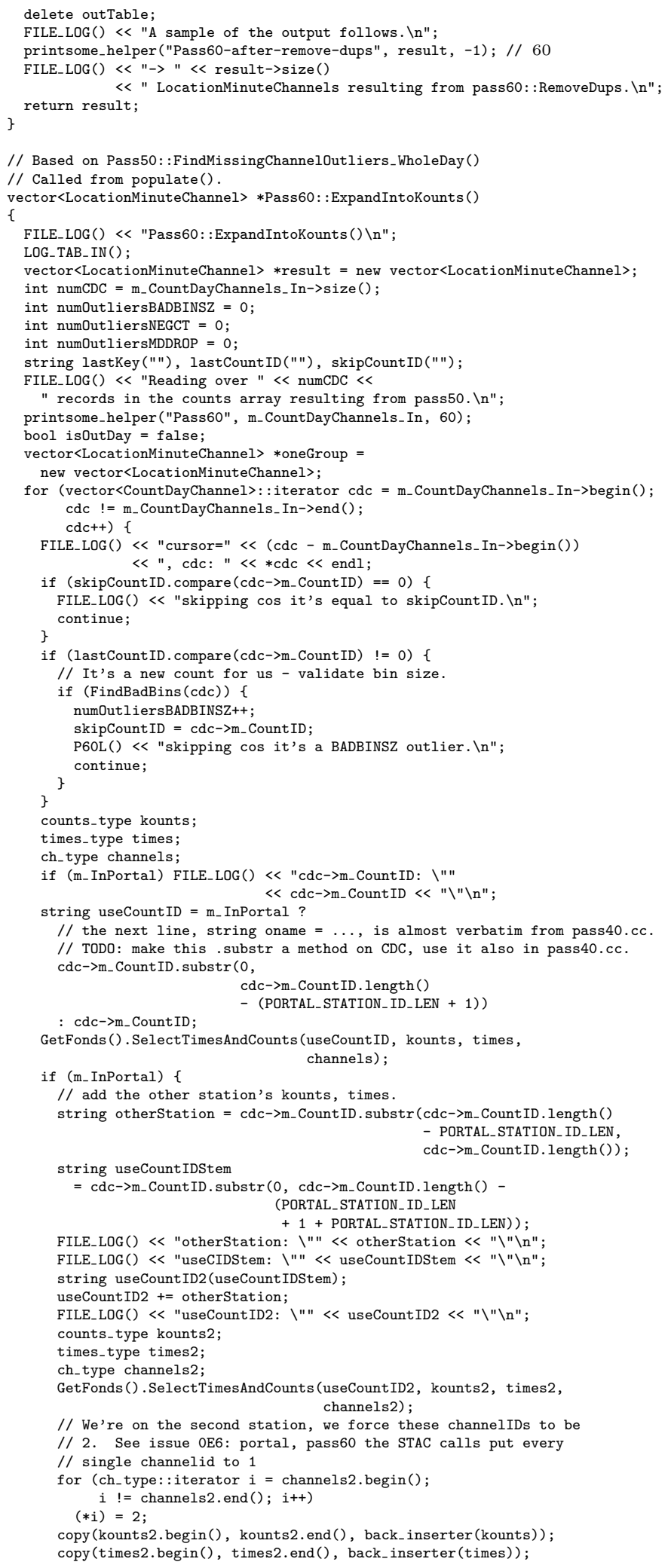




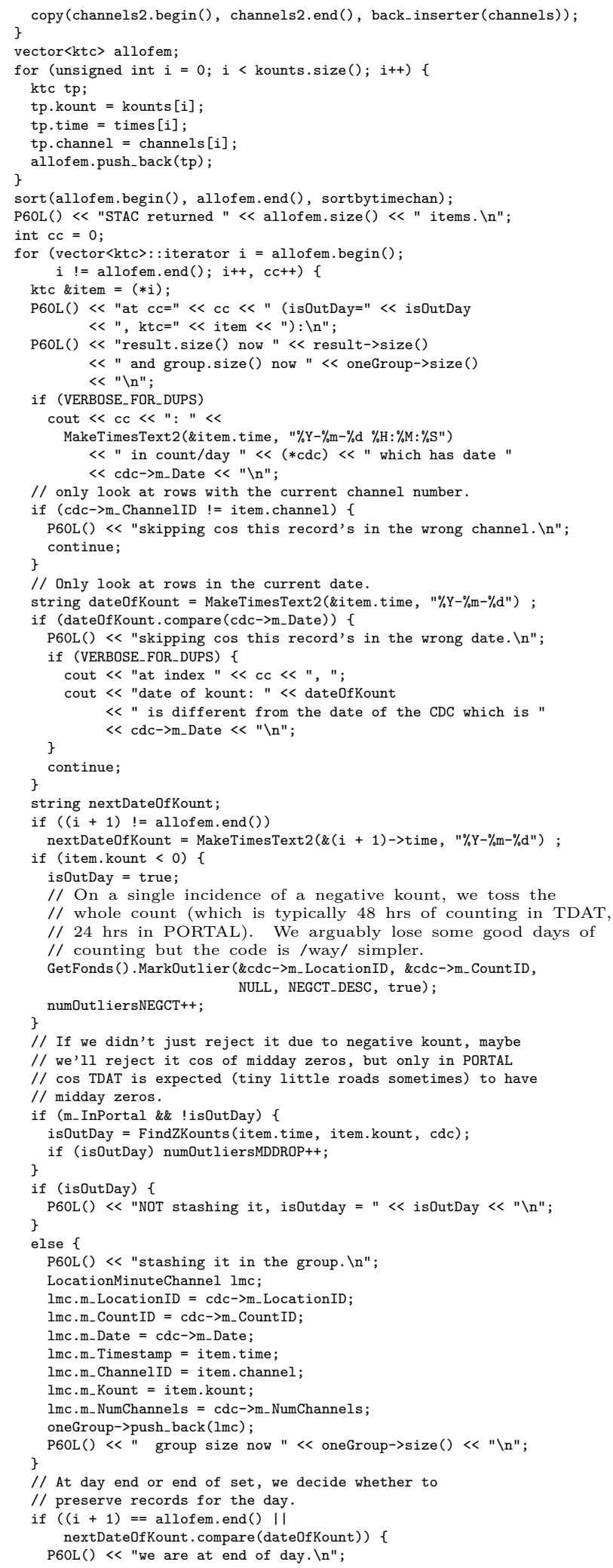




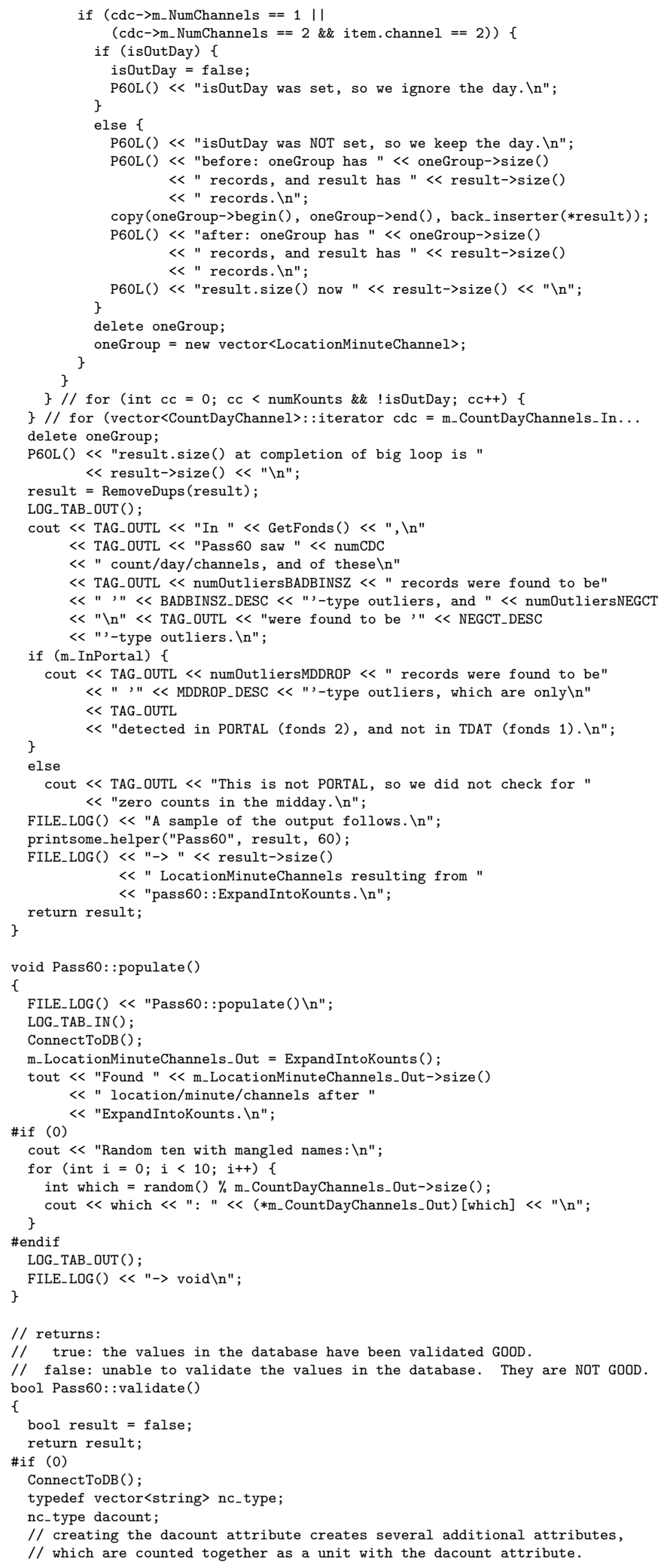




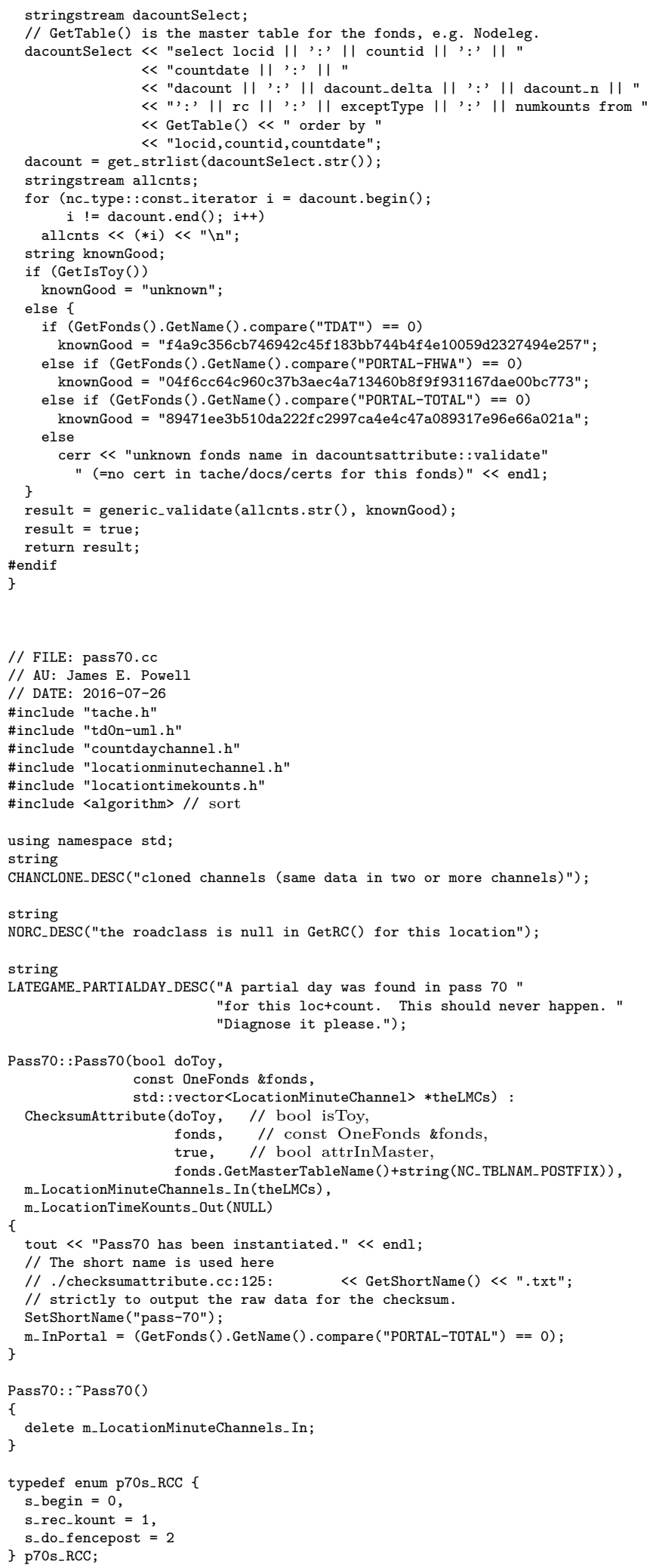




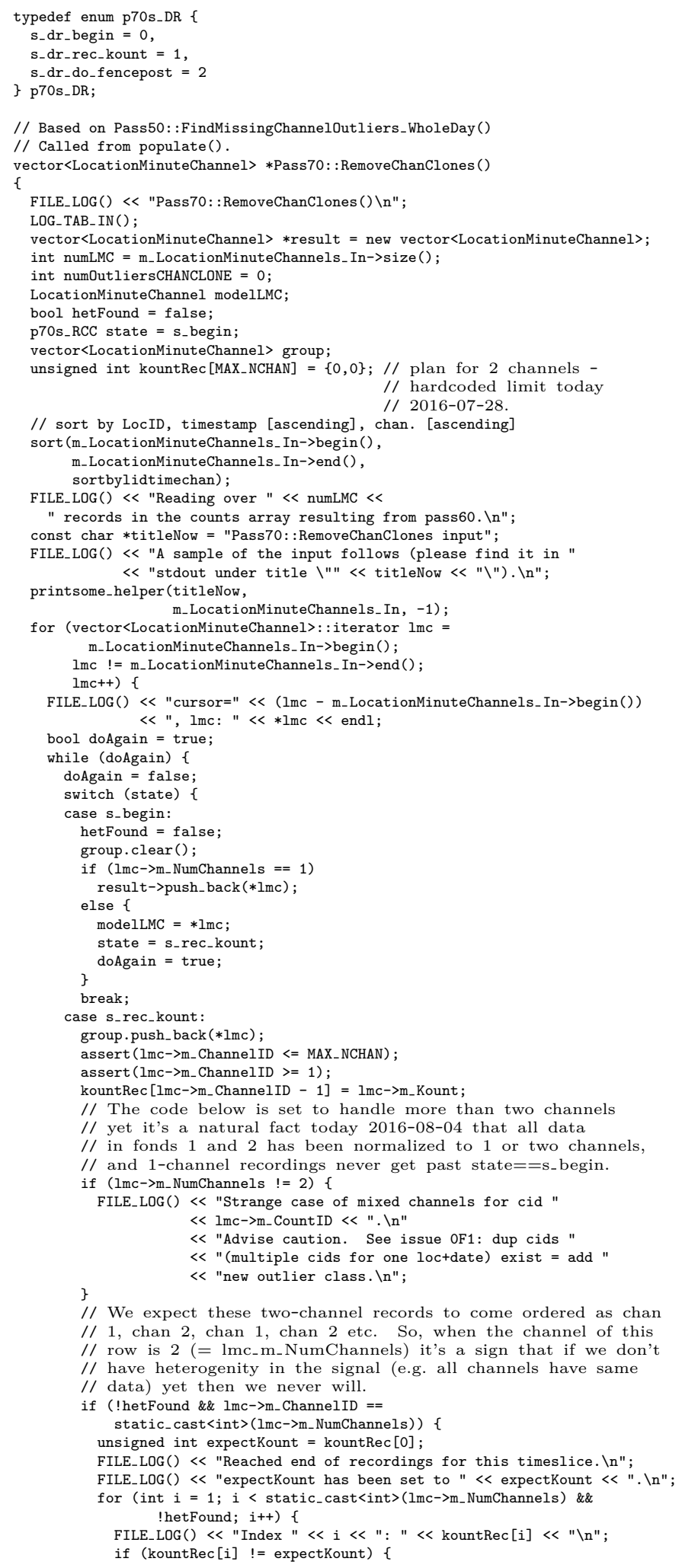

// We expect these two-channel records to come ordered as chan $/ / 1$, chan 2 , chan 1 , chan 2 etc. So, when the channel of this // row is 2 (= lmc_m_NumChannels) it's a sign that if we don't // have heterogenity in the signal (e.g. all channels have same // data) yet then we never will. 


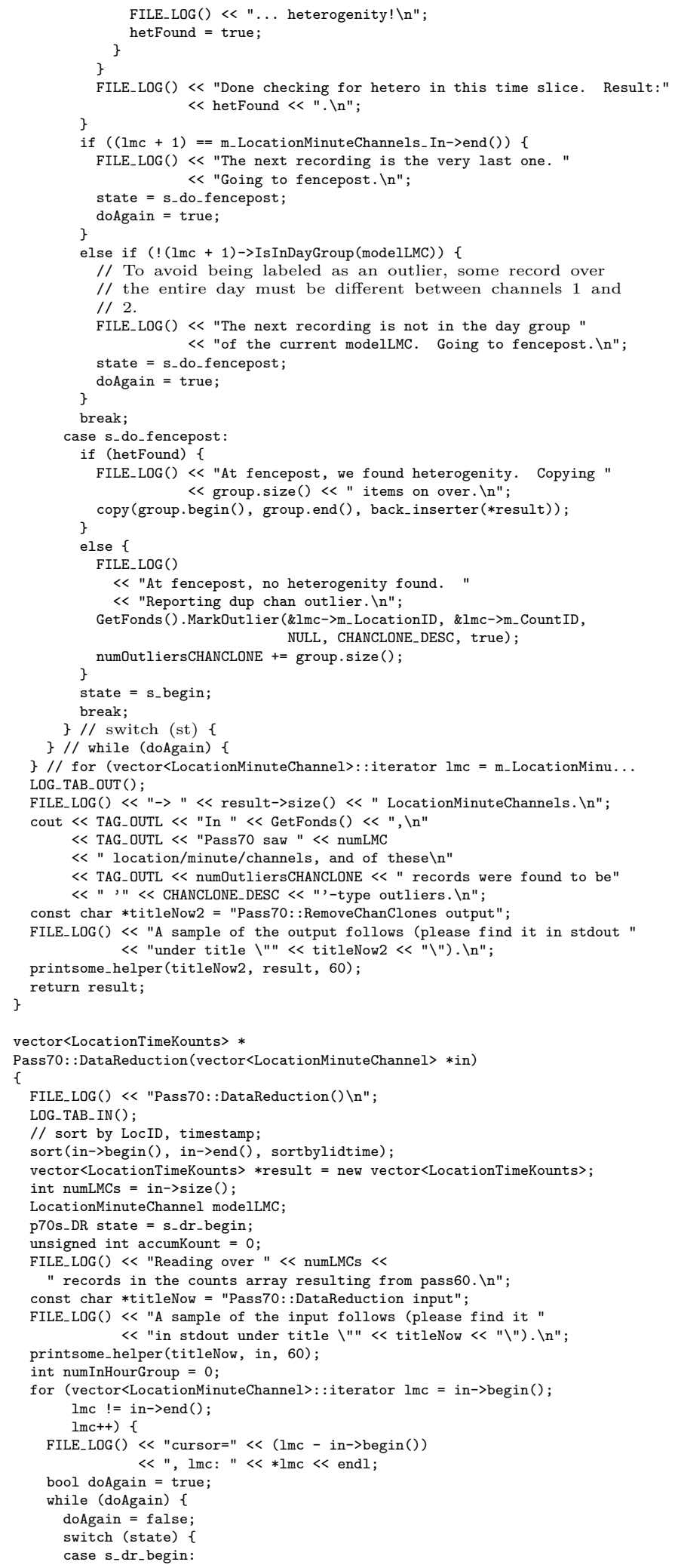




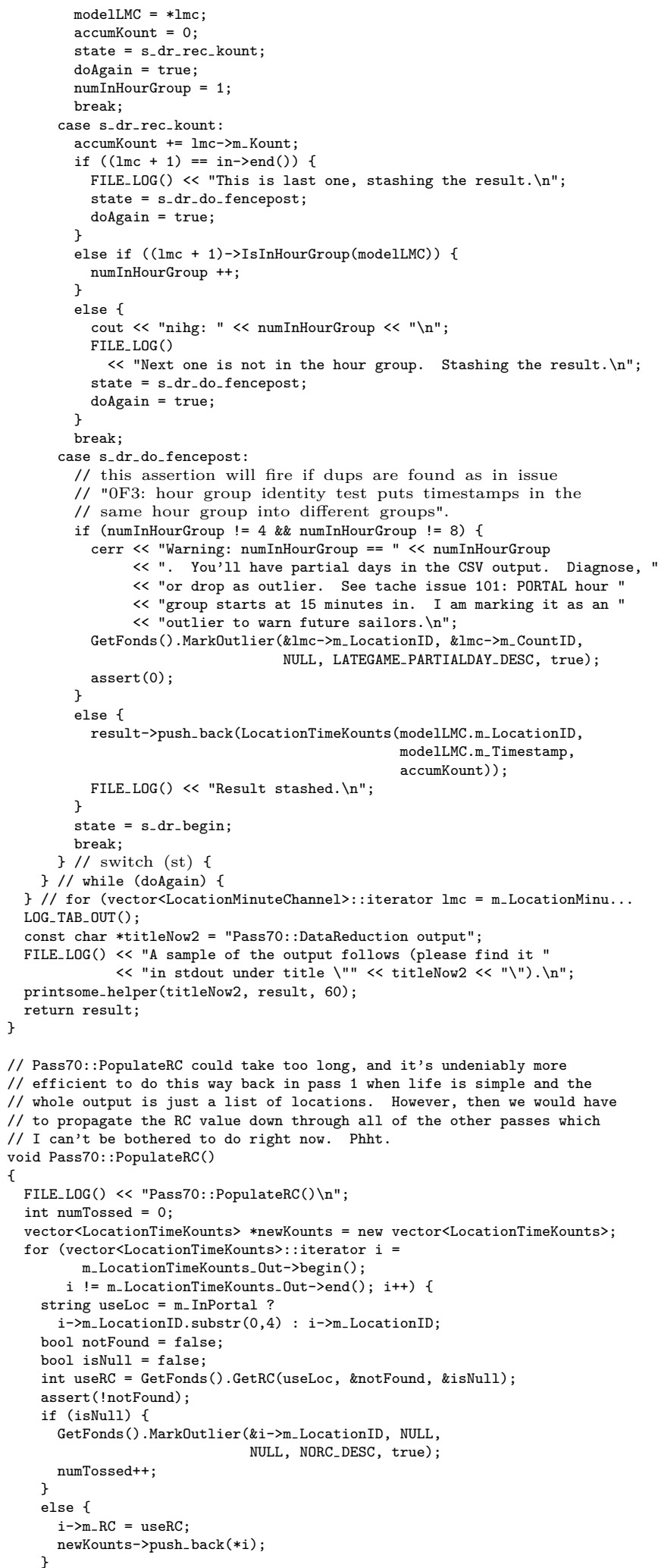




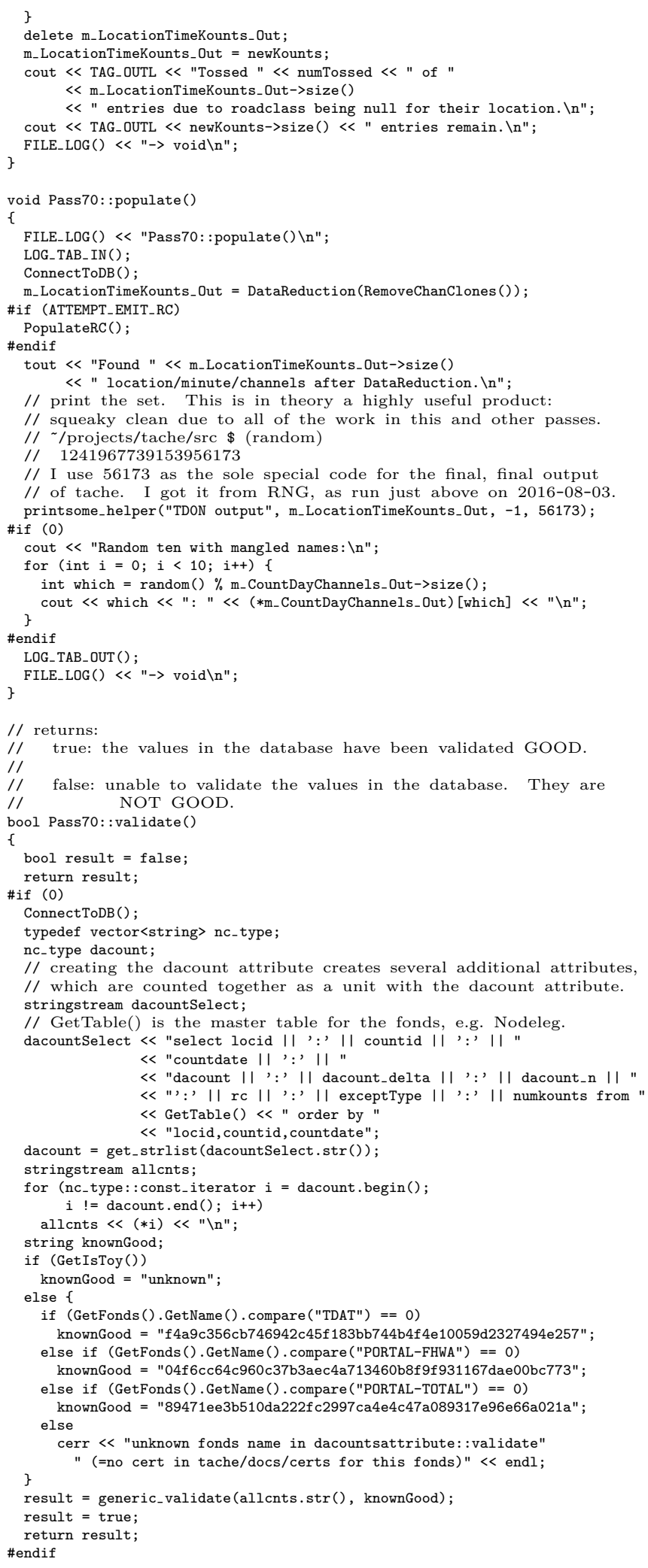


// FILE: countdaychannel.h

// AU: James E. Powell

// DATE: 2016-07-26

\#pragma once

\#include "tdon-uml.h"

// similar to inline std: :ostream\& operator<<(std: :ostream\& a,

// const LocationMinuteChannel \&b) in locationminutechannel.h

inline std::ostream\& operator $<<($ std::ostream\& $a$, const CountDayChannel \&b)

return a $\ll<$ "[a CountDayChannel with lid $=$ " $<<$ b.m_LocationID

$\ll "$ and cid " $<$ b.m_CountID $\ll$ ", date " $<$ b.m_Date

$<$ ", ChannelNo $="<<$ b.m_Channelid

$<"$ and numChan $="<<$ b.m_NumChannels $<"$ "]

// sortbylidciddatechan used in pass 50

// "comparison function object (i.e. an object that satisfies the

// requirements of Compare) which returns âẤtrue if the first argument

// is less than (i.e. is ordered before) the second" (devhelp for c++,

// deb 8 cppreference-doc-en-html)

inline bool sortbylidciddatechan(const CountDayChannel \&b1,

\{ const CountDayChannel \&b2)

// sort the input list by:

// lid + cid + date + channelno

FILE_LOG() $\ll<$ "Compare_cdc b1: " $<$ b1 $\ll$ "\n";

FILE_LOG ()$<<$ "Compare_cdc b2: " $<$ b2 $<<" \backslash n " ;$

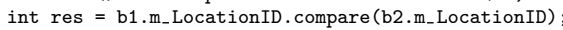

if $($ res $==0)\{$

res $=$ b1 $\cdot m_{-}$CountID . compare (b2.m_CountID)

if $(r e s==0)\{$

res $=b 1 \cdot m_{-}$Date $\cdot$ compare $\left(b 2 \cdot m_{-}\right.$Date $)$

if $($ res $==0)$ \{

$/ /$ sort in increasing order

)

res $=\mathrm{b} 1$. $\mathrm{m}_{-}$ChannelID $<\mathrm{b} 2 . \mathrm{m}_{-}$ChannelID;

\}

\}

return res >0

// FILE: locationminutechannel.h

// AU: James E. Powell

// DATE: 2016-07-26

\#pragma once

\#include "tdon-uml.h"

// similar to inline std::ostream\& operator<<(std::ostream\& a, const

// CountDayChannel \&b) in countdaychannel.h.

inline std: :ostream\& operator $<<($ std: :ostream\& a,

\{

const LocationMinuteChannel \&b)

timestamp useStamp $=$ b.m_Timestamp

return $\mathrm{a} \ll<"\left[\right.$ a LocationMinuteChannel with lid $=$ " $<$ b. $\mathrm{m}_{-}$LocationID $\ll "$ and cid " $<$ b.m_CountID $\ll$ ", date " $<$ b.m_Date $\ll<$, ChannelNo $="<<$ b.m_Channelid $<"$ " and numChan $="<<$ b.m_NumChannels $<$ ", timestamp " < MakeTimesText2 (\&useStamp, "\%Y-\%m-\%d \%H:\%M:\%S") $<$ ", kount: "

\}

$\ll$ b.m_Kount $\ll$ "] ",

// sortbylidciddatechan used in pass70 based on

// countdaychannel.h::inline bool sortbylidciddatechan(const

// CountDayChannel \&b1,...

// "comparison function object (i.e. an object that satisfies the

// requirements of Compare) which returns âẤtrue if the first argument

// is less than (i.e. is ordered before) the second" (devhelp for c++,

// deb 8 cppreference-doc-en-html)

inline bool sortbylidtime(const LocationMinuteChannel \&b1,

\{ const LocationMinuteChannel \&b2

// sort the input list by:

// lid + timestamp

\#if (0)

// (very) spammy $\rightarrow 100$ GB for a full run

FILE_LOG () $\ll$ "Compare_lmc b1: " $<$ b1 $\ll$ "\n"

FILE_LOG ()$\ll$ "Compare_lmc b2: " $<$ b2 $\ll$ "\n";

\#endif 


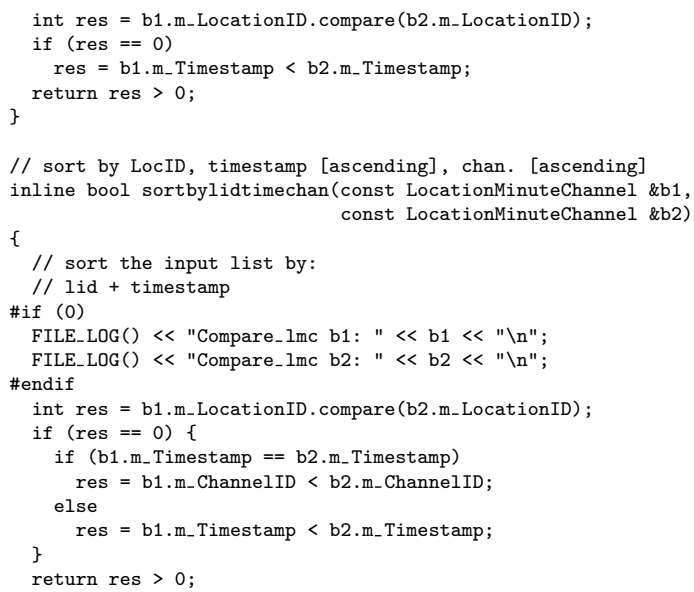




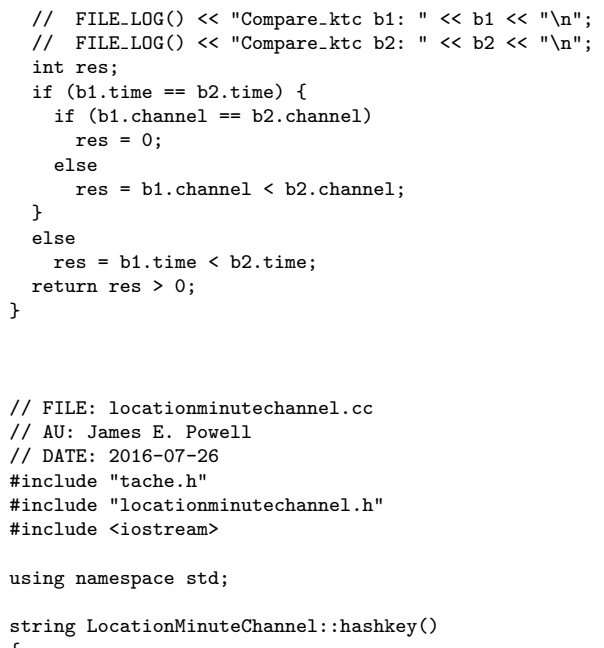


timestamp tsB = b.m_Timestamp;

string hrA = MakeTimesText2(\&tsA, "\%H");

string $\mathrm{hrB}=$ MakeTimesText2(\&tsB, $" \% \mathrm{H} ")$;

if ( $h r A . c o m p a r e(h r B)$ )

result $=$ false

LOG_TAB_OUT ();

FILE_LOG ()$<<"->$ " $<<$ result $<<" \backslash \mathrm{n} "$;

\}

return result;

// FILE: locationtimekounts.h

// AU: James E. Powell

// DATE: 2016-07-26

\#pragma once

\#include "tdon-uml.h"

inline LocationTimeKounts: :LocationTimeKounts ()\{\}

inline LocationTimeKounts::LocationTimeKounts (std: :string locID,

timestamp theTimeStamp,

unsigned int theAccumKount)

\{

m_LocationID (locID), m_Timestamp (theTimeStamp), m_Kounts (theAccumKount)

// similar to inline std::ostream\& operator<<(std::ostream\& a, const

// CountDayChannel \&b) in countdaychannel.h.

inline std::ostream\& operator $<$ (std: :ostream\& a,

$\{$ timestamp useStamp $=$ b.m_Timestamp;

\#if $(0)$

return a

$<<$ "[a LocationTimeKounts with lid $=$ ")

$<$ b.m_LocationID

$<<$ ", timestamp "

< MakeTimesText2(\&useStamp, "\%Y-\%m-\%d \%H:\%M:\%S")

$<<$ ", kounts: "

$<$ b. m_Kounts $<"$ "] "

$\#$ else

//

return a

$<$ b.m_LocationID

\#if (ATTEMPT_EMIT_RC)

$<$ ", "

$<$ b. m_ RC

\#endif

$<$ " , "

< MakeTimesText2 (\&useStamp, "\%Y-\%m-\%d \%H:\%M:\%S")

$<<"$ ",

$<$ b.m_Kounts $<<$ "\n";

\#endif 